
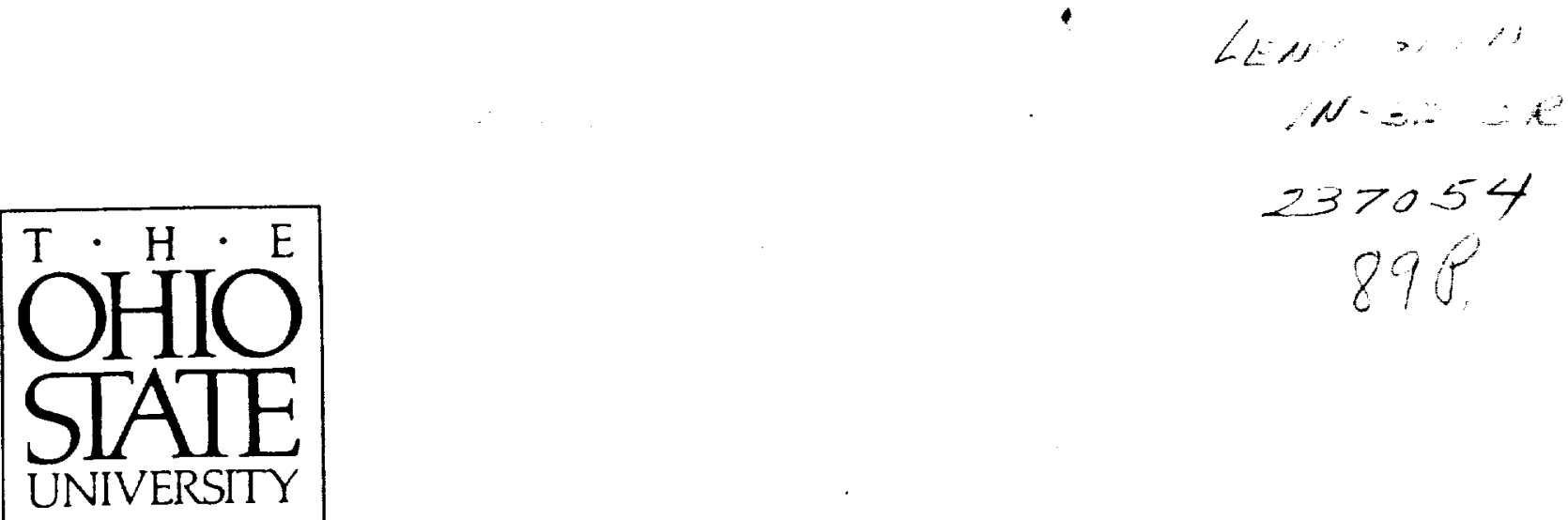

\title{
An Experimental SMI Adaptive Antenna Array for Weak Interfering Signals
}

R.L. Dilsavor and I.J. Gupta

\section{The Ohio State University \\ ElectroScience Laboratory \\ Deportment of Eloctrical Engineering \\ Columbus, Ohio 43212}

Technical Report 716111-7

Grant No. NAG3-536

October 1989

National Aeronautics and Space Administration

Lewis Research Center

21000 Brookpark Road

Cleveland, Ohio 44135

(NASA-CR-185976) AN FXPERIMENTAL SMI

ARAPIIVE ANTENNA ARRAY FOR WLAK INIFREEPING

$\mathrm{N}^{\circ} \mathrm{O}-112 ! 1$

SIGNALS

(Ohio State Univ.)

ag $\mathrm{C}$ CSCL 179

r.j/32 Unclas 0237054 


\section{NOTICES}

When Government drawings, specifications, or other data are used for any purpose other than in connection with a definitely related Government procurement operation, the United States Government thereby incurs no responsibility nor any obligation whatsoever, and the fact that the Government may have formulated, furnished, or in any way supplied the said drawings, specifications, or other data, is not to be regarded by implication or otherwise as in any manner licensing the holder or any other person or corporation, or conveying any rights or permission to manufacture, use, or sell any patented invention that may in any way be related thereto. 


\begin{tabular}{|c|c|c|c|}
\hline $\begin{array}{l}\text { REPORT DOCUMENTATION } \\
\text { PAGE }\end{array}$ & 1. REPORT NO. & 2. & 3. Reclpient's Accession No. \\
\hline \multirow{2}{*}{\multicolumn{2}{|c|}{$\begin{array}{l}\text { 4. Title and Subtitle } \\
\text { An Experimental SMI Adaptive Antenna Array } \\
\text { for Weak Interfering Signals }\end{array}$}} & & $\begin{array}{l}\text { s. Report Date } \\
\text { October } 1989 \\
\end{array}$ \\
\hline & & & o. \\
\hline \multicolumn{3}{|c|}{$\begin{array}{l}\text { T. Author(s) } \\
\text { R.L. Dilsavor and I.J. Gupta }\end{array}$} & $\begin{array}{l}\text { 8. Performing Org. Hept. No. } \\
716111-7 \\
\end{array}$ \\
\hline & 10. Project/Task/Work Unlt No. \\
\hline $\begin{array}{l}\text { ElectroScience Laboratory } \\
1320 \text { Kinnear Road } \\
\text { Columbus, OH } 43212 \\
\end{array}$ & & & $\begin{array}{l}\text { 11. Contract(C) or Grant(G) No. } \\
\text { (C) } \\
\text { (G) NAG s-sso }\end{array}$ \\
\hline \multirow{2}{*}{\multicolumn{3}{|c|}{$\begin{array}{l}\text { 12. Sponsoring Organization Name and Address } \\
\text { National Aeronautics and Space Administration } \\
\text { Lewis Research Center, } 21000 \text { Brookpark Road }\end{array}$}} & $\begin{array}{l}\text { 13. Report Type/Perlod Covered } \\
\text { Technical Report }\end{array}$ \\
\hline & & & 14. \\
\hline
\end{tabular}

15. Supplementary Notes

16. Abstract (Limit: 200 words)

A modified SMI algorithm designed to increase the suppression of weak interference is implemented on an existing experimental array system. The algorithm itself is fully described as are a number of issues concerning its implementation and evaluation, such as sample scaling, snapshot formation, weight normalization, power calculation, and system calibration. Several experiments show that the "steady state" performance (i.e. many snapshots are used to calculate the array weights) of the experimental system compares favorably with its theoretical performance. It is demonstrated that standard SMI does not yield adequate suppression of weak interference. Modified SMI is then used to experimentally increase this suppression by as much as $13 \mathrm{~dB}$.

17. Document Analysis a. Descriptors

b. Identiflers/Open-Ended Terms

c. COSATI Field/Group

16. Availability Statement

A. Approved for public release; Distribution is unlimited.
18. Security Class (Thls Report) Unclassified

20. Security Class (This Page) Unclassified 


\section{Contents}

1 Introduction

2 The Original Experimental System 6

2.1 Array Simulator . . . . . . . . . . . . 7

2.2 Array Processor . . . . . . . . . . . . . . 12

2.3 Signal Simulator . . . . . . . . . . . . 14

3 Experimental Modifled SMI System 17

3.1 A Hardware Change in the Original System . . . . . . . 17

3.2 Sampling and Weighting the Main Channel ... . . . . 19

3.3 Scaling the Samples . . . . . . . . . . . . . 19

3.4 The Modified SMI Algorithm . . . . . . . . . . . . 21

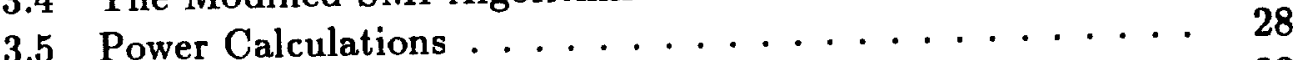

3.6 System Calibration . . . . . . . . . . . . . 33

3.7 Estimation of the Steering Vector . . . . . . . . 38

4 Experimental Results 40

4.1 Experiment 1: Two elements, one interference, standard SMI, vary interference power . . . . . . . . . . . 42

4.2 Experiment 2: Three elements, one interference, desired signal, standard SMI, vary interference arrival angle . . . . . . 44

4.3 Experiment 3: Three elements, one interference, desired signal, standard SMI, vary interference power . . . . . . 46

4.4 Experiment 4: Three elements, one interference, desired signal, standard SMI, vary main antenna sidelobe . . . . . . 
4.5 Experiments 5 and 6: Three elements, one interference, desired signal, fixed input powers, modified SMI, vary fraction

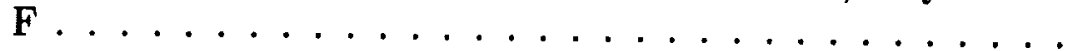

5 Conclusions

A Experimental System Software (Fortran IV) 


\section{List of Figures}

1.1 An $\mathrm{N}$-element array receiving a desired signal and $\mathrm{M}$ inter-

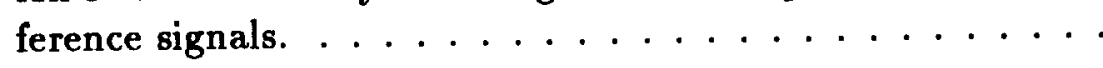

2.1 Modified feedback loop that uses separate antennas and amplifiers to decorrelate noise at the correlator inputs. . . . . 8

2.2 Block diagram of the experimental system. . . . . . . . 9

2.3 Array simulator; detailed block diagram. . . . . . . . . . . 11

2.4 Array Processor; detailed block diagram. . . . . . . . . . 13

2.5 Staggered Pulse modulation of the desired and two interference signals. . . . . . . . . . . . . . 16

3.1 Comparison of the ideal array and our experimental system. 22

3.2 Typical I and Q VDM outputs. . . . . . . . . . . 30

4.1 Experiment 1: Suppression of a single interference of varying power with a two-element array. ...........

4.2 Experiment 2: Suppression of a single interference of varying arrival angle with a 3 -element array and $S N R$ (main) $=16 \mathrm{~dB}$.

4.3 Experiment 3: Suppression of the first interference signal with a 3 -element array, $\operatorname{SNR}($ main $)=16.5 \mathrm{~dB}$. The top pair of curves are theory and experiment for fixed INR(aux1) $\operatorname{INR}($ main $)=9.7 \mathrm{~dB}$. The bottom pair are for INR(aux1) $\operatorname{INR}($ main $)=6.5 \mathrm{~dB} \ldots \ldots \ldots \ldots$

4.4 SNR plots for Experiment 3: The top three curves are input SNR(main), theoretical SNR(out), and experimental SNR(out) for INR(aux1) $=\operatorname{INR}($ main $)+6.5 \mathrm{~dB}$. The bottom three curves are the same for $\operatorname{INR}(\operatorname{aux} 1)=\operatorname{INR}($ main $)+9.7 \mathrm{~dB}$ and have been displaced $-4 \mathrm{~dB}$ for clarity. . . . . . . . . . . 
4.5 Experiment 4: Suppression of the first interference signal with a 3-element array, $\mathrm{SNR}$ (main) $=16.06 \mathrm{~dB}$. The first auxiliary element gain is fixed and the main element sidelobe varies.

4.6 SNR(main) and SNR(out) curves for Experiment 4. . . .

4.7 Experiment 5: Suppression of the first interference signal versus fraction $F$ using a 3-element modified SMI array with desired SNR(main $)=16.50 \mathrm{~dB}$. INR(main $)=-9.45 \mathrm{~dB}$. Bottom pair of curves has INR(aux1) $=-3.57 \mathrm{~dB}$. Top pair has $\operatorname{INR}(\operatorname{aux} 1)=0.43 \mathrm{~dB} . \operatorname{INR}(\operatorname{aux} 2)=-20 \mathrm{~dB} . \ldots \ldots . .$.

4.8 SNR curves for Experiment 5. Top three curves are input SNR(main), theoretical SNR(out), experimental SNR(out) for INR $(\operatorname{aux} 1)=-3.57 \mathrm{~dB}$. Similarly, the bottom three curves are for $\operatorname{INR}(\operatorname{aux} 1)=0.43 \mathrm{~dB}$ and have been displaced $-4 \mathrm{~dB}$ for clarity. . . . . . . . . . . . . . . . . .

4.9 Experiment 6: Suppression of the first interference signal versus fraction $\mathrm{F}$ using a 3-element modified SMI array with desired $\operatorname{SNR}($ main $)=16.50 \mathrm{~dB} . \operatorname{INR}($ main $)=-2.68 \mathrm{~dB}$. Bottom pair of curves has INR(aux1) $=0.53 \mathrm{~dB}$. Top pair has INR(aux1) $=4.72 \mathrm{~dB} . \operatorname{INR}(\operatorname{aux} 2)=-17 \mathrm{~dB} \ldots \ldots \ldots \ldots$

4.10 SNR curves for Experiment 6. Top three curves are input SNR(main), theoretical SNR(out), experimental SNR(out) for INR $(\operatorname{aux} 1)=0.53 \mathrm{~dB}$. Similarly, the bottom three curves are for $\operatorname{INR}(\operatorname{aux} 1)=4.72 \mathrm{~dB}$ and have been displaced $-4 \mathrm{~dB}$ for clarity. . . . . . . . . . . . . . . . . 


\section{Chapter 1}

\section{Introduction}

Adaptive antenna arrays have time-variable, controllable array patterns which enables them to suppress interference from moving sources of interference while receiving a desired signal whose source may also be moving. The output of an adaptive array is the sum of the weighted (in amplitude and phase) outputs of a number of antennas or array elements as shown in Figure 1.1. The pattern of the array at any time is a function of the weight values at that time. The weights may be controlled according to some closed-loop feedback control law as in the LMS array or Applebaum array or they may be varied according to an open-loop weight assignment rule as in the SMI array [1]. In any case, an array must be given information that allows it to distinguish the desired signal from the interference signals to be suppressed. For the Applebaum and SMI arrays, the information consists of the desired signal direction, whereas for the LMS array, a reference signal that is correlated with the desired signal must be derived. Given good information about the desired signal, each of the three array types mentioned above is known to maximize the output signal-to- 


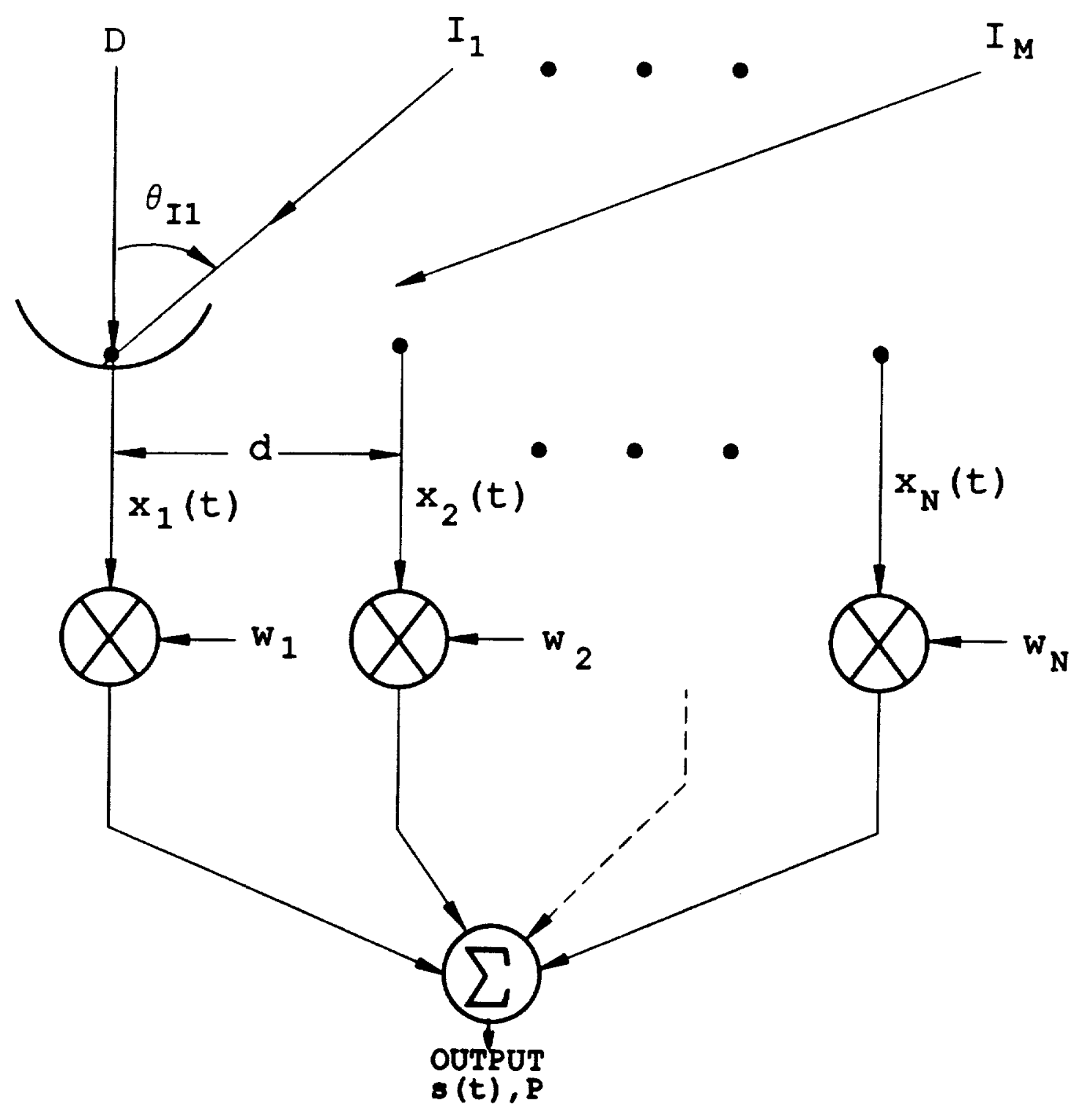

Figure 1.1: An N-element array receiving a desired signal and $\mathrm{M}$ interference signals. 
interference-plus-noise ratio (SINR) under steady state conditions [1]. As a result, when the interference power is weak compared to the noise power the weights adapt to maximize the signal-to-noise ratio and the interference is left more or less unsuppressed.

The problem of suppressing weak interference has been recently addressed by Gupta and Ksienski [2] who proposed a hardware modification of the feedback loops which control the array weights. In this modification, two spatially separate antennas followed by their individual amplifiers are used with each feedback loop to yield decorrelated noise at the inputs of the feedback loop correlators. It was shown that, in theory, the modified feedback loops can provide the required interference suppression. Ward et. al. $([3],[4])$ built an experimental adaptive array and verified the theoretical analysis.

Later, as an alternative, Gupta [5] proposed a software modification of the SMI array to enhance weak interference suppression. In the modified SMI algorithm the sample covariance matrix is redefined to subtract out the effects of the thermal noise. This is done by subtracting a fraction $F$ of the minimum eigenvalue of the original covariance matrix from its diagonal elements. This step is justified by noting that, in theory, the only effect of thermal noise (assuming the noise is uncorrelated, zero mean, and has equal power $\sigma^{2}$ at each array element) on the covariance matrix is an additive $\sigma^{2}$ term on each diagonal element of the true covariance matrix. Furthermore, if the number of elements in the array is greater than the number of signals incident on the array, the minimum eigenvalue of the sample covariance matrix is an estimate of the noise power $\sigma^{2}$. Gupta [5] 
showed that, in theory, the modified SMI algorithm provides the required interference suppression assuming the true covariance matrix is known.

In normal applications, the true covariance matrix is not known a priori due to incomplete knowledge of the receive antenna patterns and received signal characteristics. As a result, samples of the received signals (i.e. snapshots) are used to estimate the covariance matrix which, in turn, is used in the weight calculation. Dilsavor and Moses [6] have addressed the question of how many snapshots are needed to achieve good estimates of the modified SMI weights. Using Monte Carlo simulations and statistical theory, they found that the number of snapshots required for good estimates of the modified SMI weights increases with the amount of diagonal subtraction (i.e. with the fraction F). Thus, a tradeoff was found to exist between the improved suppression of weak interference provided by the modified SMI algorithm and the number of snapshots required to achieve that improvement.

The modified SMI algorithm has been implemented on the aforementioned experimental array built by Ward et.al. The experimental SMI array is fully adaptive with either two or three elements. It can simulate narrowband signal scenarios consisting of one desired signal arriving from broadside and up to two interference signals arriving from arbitrary directions. Since the original system built by Ward was a modified Applebaum array in the sidelobe canceller configuration, some hardware as well as software alterations were necessary in implementing the modified SMI algorithm. The purpose of this report is to describe the implementation of the modified SMI algorithm on the original experimental system and then to study 
the performance of the experimental SMI system in comparison with theory. The rest of the report is organized as follows. Chapter 2 offers a brief description of the original experimental system built by Ward. Chapter 3 describes the implementation of SMI on the original system and includes a description of hardware modifications, sampling procedures, the implemented algorithm, weight normalization, performance evaluation, and system calibration. Experiments conducted to evaluate the performance of the experimental system are described in Chapter 4 . Chapter 5 contains our conclusions. Finally, the system software is included as an appendix. 


\section{Chapter 2}

\section{The Original Experimental System}

The experimental system built by Ward, et. al. $([3],[4])$ is a sidelobe canceller with an unweighted main element and two weighted auxiliary elements which employs a discrete version of the Applebaum algorithm for weight control. The signal scenarios which can be tested consist of a desired signal arriving from broadside and up to two interference signals arriving from arbitrary directions.

The system is designed to simulate the array whose auxiliary element weights are controlled by the modified feedback loops of Figure 2.1. In these modified feedback loops the effect of noise on the array weights is decreased by reducing the correlation between the noise components of the two inputs to the correlator. The reduction in correlation is achieved by using two spatially separate antennas, each followed by its own amplifier, with each auxiliary element as shown in Figure 2.1. In this configuration, the "3-element" array actually uses 5 antennas; one for the unweighted main element and two for each of the weighted auxiliary elements. For purposes 
of parameter control and system performance evaluation, the experimental system can simulate the signals that would be received by the five antennas. Desired, interference, and noise signals are bench generated and combined in an array simulator to simulate the signals received by the five antennas. The experimental adaptive processor can also be used with signals received from geosynchronous satellites. In this case, the signals received by actual antenna elements are downconverted to $69 \mathrm{MHz}$, the center frequency of the adaptive processor, and then fed to the experimental system. In the results presented in this document, the simulated signals are used.

A block diagram of the experimental system is given in Figure 2.2. The signal simulator generates a desired signal and two interference signals. In the array simulator, these signals are combined with each other and with noise to form the signals that would be received at the 5 antennas. The array processor applies weights to the auxiliary element signals and forms (by summing) the adapted array output. The system operates at $69 \mathrm{MHz}$ with a bandwidth of $6 \mathrm{MHz}$. A digital computer (PDP 11/23) is used to implement the weight control algorithm, control the various components, and evaluate the system performance. A description of the individual system blocks is given below.

\subsection{Array Simulator}

Figure 2.3 shows a detailed block diagram of the array simulator. In the array simulator, the incident signals are combined and thermal noise is added to form the signals received at each array element, such that each 


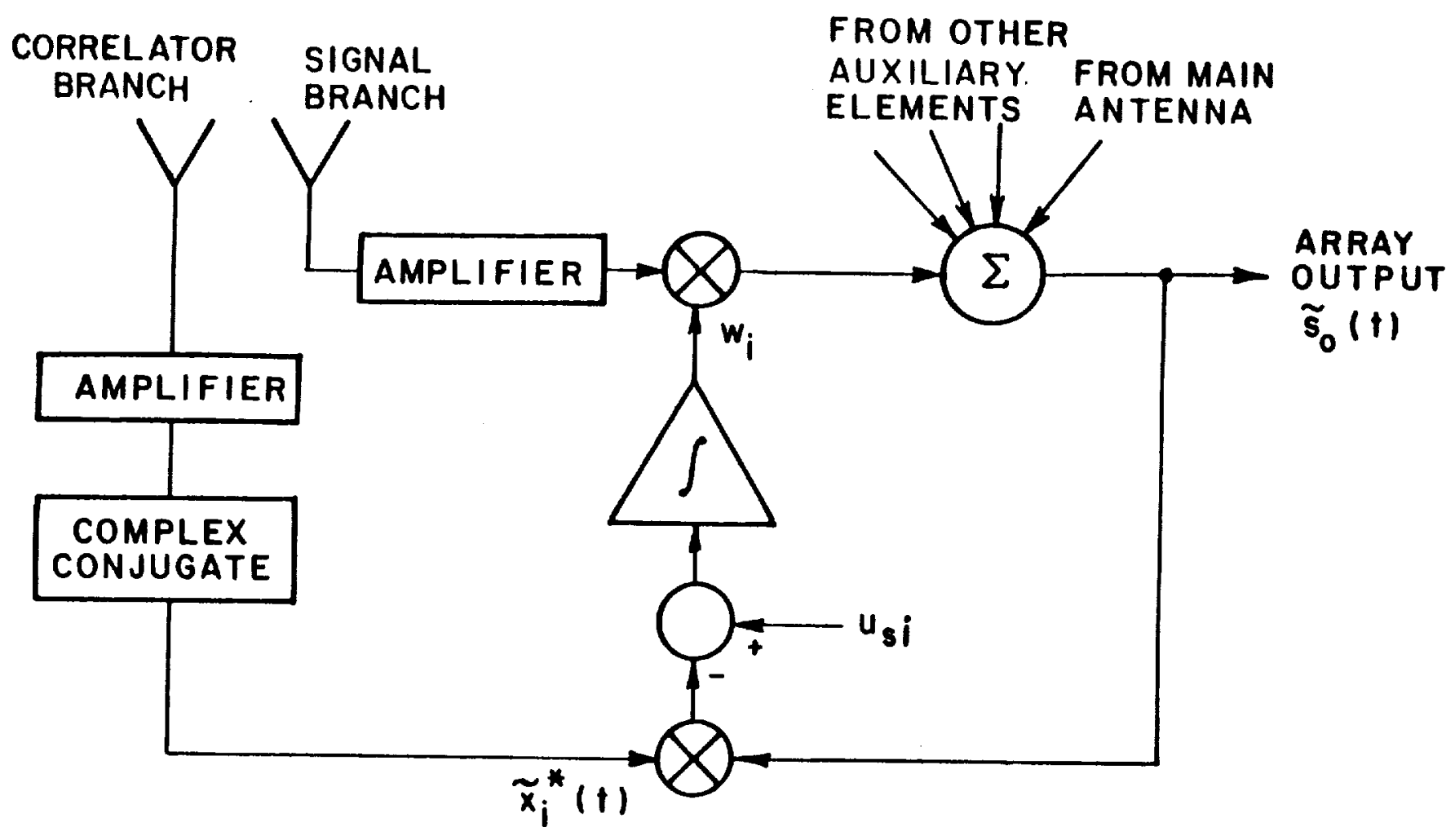

Figure 2.1: Modified feedback loop that uses separate antennas and amplifiers to decorrelate noise at the correlator inputs. 


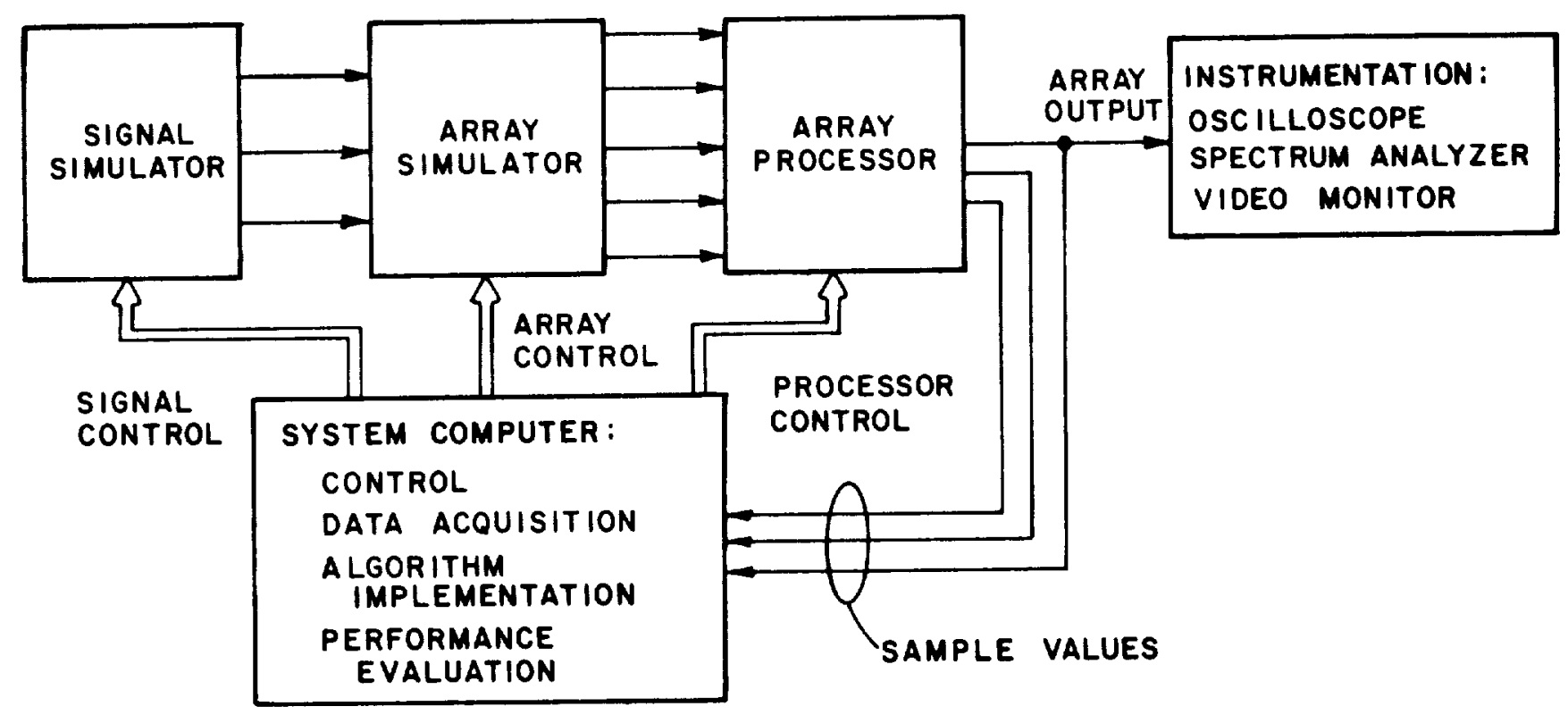

Figure 2.2: Block diagram of the experimental system. 
signal contains a component due to the desired signal, components due to one or both interfering signals, and additive thermal noise. Thus the array simulator has three inputs for the three incident signals, and five outputs corresponding to the five antennas of the array. These five outputs are designated MAIN, AUX-1 SIGNAL, AUX-1 CORRELATOR, AUX-2 SIGNAL, AND AUX-2 CORRELATOR. The MAIN output is the signal received by the main element of our array.

The other outputs are the signals received by the two auxiliary elements of our sidelobe canceller with modified feedback loops. The designations SIGNAL and CORRELATOR specify the two branches of the modified feedback loop (Figure 2.1) of a particular auxiliary element. The SIGNAL branch is weighted and proceeds to the array output summer, whereas the CORRELATOR branch forms the input to the correlator. Note in Figure 2.3 that the noise components injected into the auxiliary SIGNAL branches and the MAIN channel are all from different noise sources, and thus are uncorrelated. Furthermore, the noise components in the auxiliary CORRELATOR branches originate from another noise source, and are therefore uncorrelated with the noise components of the SIGNAL branches. In Figure 2.3 the $\Delta$ 's are zero-phase power dividers connected as summers. The $\alpha$ 's are variable attenuators and the $\phi$ 's denote variable phase shifters. $N_{1}$ through $\mathrm{N}_{4}$ are the noise sources. The phase shifters simulate variations of the interfering signal directions of arrival by varying the interelement phase shifts between interfering signal components of different array elements . There are no phase shifters associated with the desired signal because it is assumed to arrive from broadside and thus is received with the same phase 


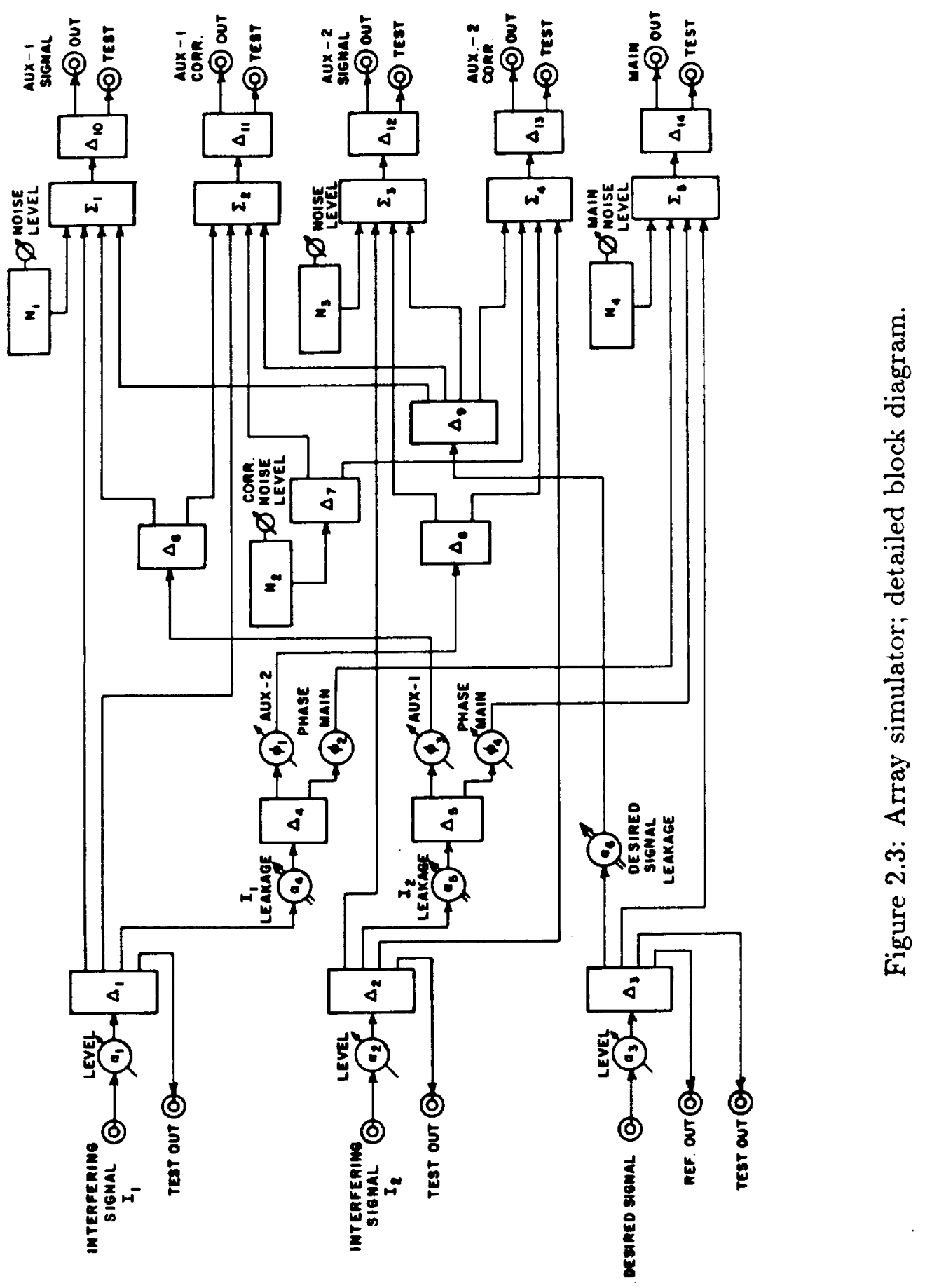


at each array element. Variable attenuators are used to control the amount of each incident signal received at each output channel. This is analogous to varying the gains of the main and auxiliary elements in the directions of the incident signals. Once the desired signal scenario is set, the array simulator outputs are fed to the array processor, where the signals are sampled and the auxiliary element weights are determined by digital computer and are implemented in analng. The array processor is discussed next.

\subsection{Array Processor}

Together with the system computer, the array processor forms the two modified feedback loops of the sidelobe canceller, through which the weight control algorithms are implemented. A detailed block diagram of the array processor is shown in Figure 2.4. Note that the auxiliary channel correlator branch signals are down converted to baseband and quadrature detected by the vector demodulators (VDMs), as is the array output. These baseband voltages are simultaneously sampled, analog-to-digital (A/D) converted and read by the system computer which implements the weight control equation and calculates the array weights. The new weights are then (D/A) converted and applied to the auxiliary element SIGNAL branches as in-

phase (I) and quadrature (Q) control voltages by the two vector modulators (VMODs). The weighted auxiliary elements are then summed with the main channel signal to form the array output.

In the array processor, the $I$ and $Q$ outputs of each vector demodulator are processed prior to being sampled. A low pass filter first removes the 


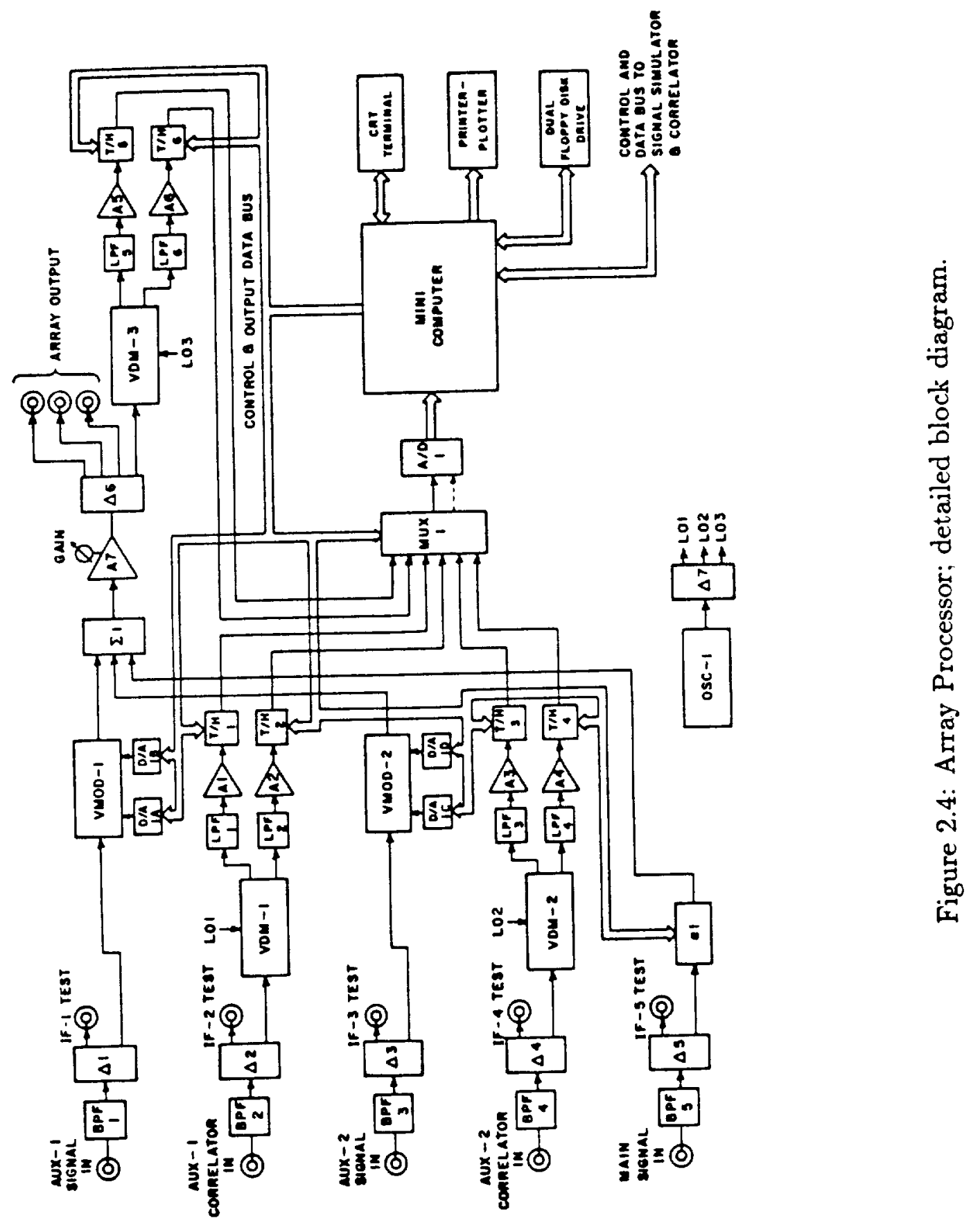


second harmonic. The resultant baseband signals are then amplified to utilize the full dynamic range of the A/D converter. Track and hold devices allow the multiplexing of all six VDM outputs to the single A/D converter , so that they can be sampled simultaneously. This process preserves the signal correlation and noise correlation (if any) between the samples of different channels. For the experiments to be described here, the inputs to the processor are the signals from the array simulator. However, this same array processor may be used with signals from an actual antenna array, after downconversion to the array processor center frequency of $69 \mathrm{MHz}$ [7]. The signals provided to the array simulator are described next.

\subsection{Signal Simulator}

The signal simulator synthesizes the desired signal and the two interfering signals, which are then combined in the array simulator to form the received signals at each array element. In order to measure adaptive array performance characteristics such as interference suppression, output interference-to-noise ratio (INR), and output signal-to-interference-plusnoise ratio (SINR), it is necessary to measure separately the desired signal power, the interference signal power, and the noise power present in each of the array elements and in the array output. Pulse modulated sinusoids are used as the desired signal and the interfering signals to accomplish this objective. As shown in Figure 2.5, the modulation on one interfering signal is staggered from the modulation on the other interfering signal, and from the desired signal modulation, such that the signal occupies a different por- 
tion of the pulse repetition period. There is also a portion of the pulse period when only noise (no signal) is present. The desired and interfering signals are therefore all uncorrelated with each other (for all interelement time delays of interest). The track/hold devices of the array processor are triggered in synchronism with this modulation envelope. Because of $A / D$ conversion speed limitations, successive samples are not taken in the same waveform period. Instead, each sample is from a different pulse repetition period, but is separated by only a small time interval from the point on the waveform at which the previous sample was taken. Thus, an effective sampling rate much higher than the sampling rate possible in real time is achieved. By varying the delay from the start of a period to a sampling instant, a sequence of samples covering the entire waveform is provided to the system computer. The levels of a particular signal component (desired, interference, or noise) are obtained by signal averaging over that portion of the pulse repetition period corresponding to the signal component under measurement. In this manner, the sampled data and the computer are also used for steady state adaptive array performance evaluation. However, as discussed in the next chapter, this pulse modulation scheme is exploited solely for performance evaluation and not in determining the auxiliary element weights. The experimental modified SMI system is described next. 


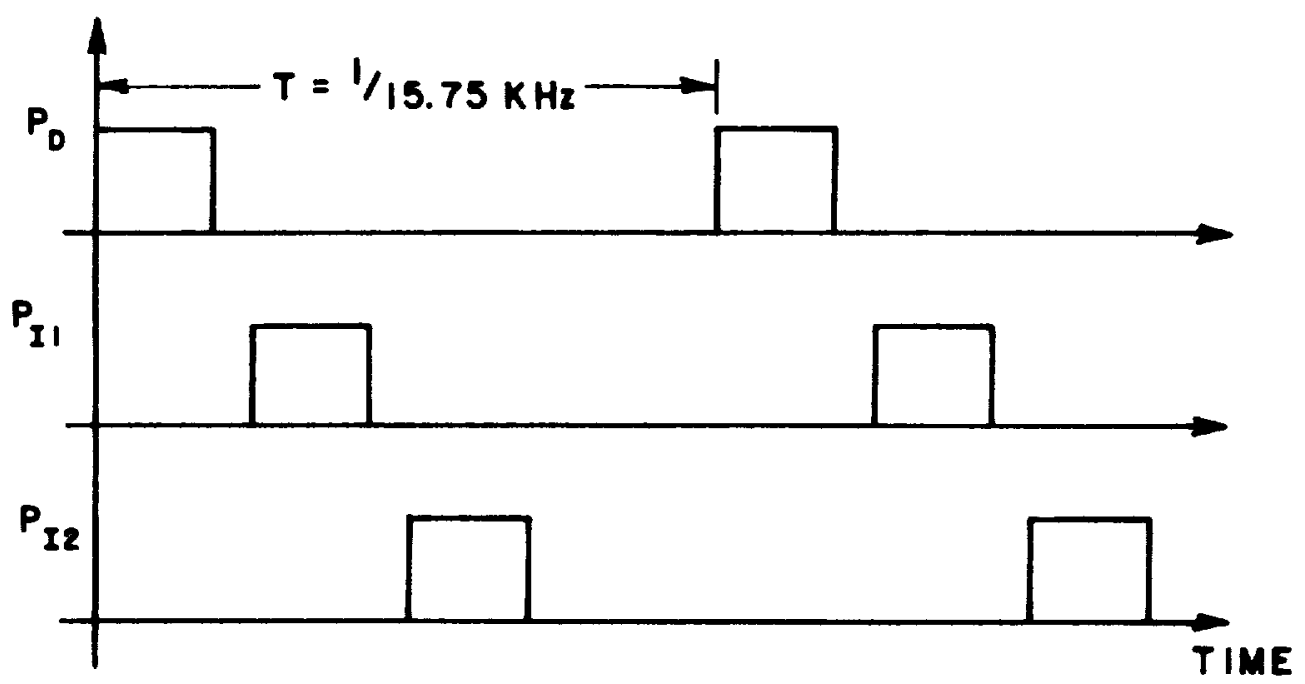

Figure 2.5: Staggered Pulse modulation of the desired and two interference signals. 


\section{Chapter 3}

\section{Experimental Modified SMI System}

A number of issues were addressed in implementing the two- or threeelement fully adaptive modified SMI array on the original system. These issues as well as the modified SMI algorithm itself are described in this chapter.

\subsection{A Hardware Change in the Original Sys- tem}

The modified SMI system represents a software solution to the problem of weak interference suppression, whereas the original system embodies a hardware solution (modified feedback loops) to the same problem. Originally two antennas and amplifiers were used with each feedback loop in order to decorrelate the noise components of the two signals at each feedback loop correlator input. The original system modeled this situation by using different noise sources to generate the noise components. Separate 
noise sources for the SIGNAL and CORRELATOR branches of each element of the array processor are not needed in the SMI system since this system does not need two separate antennas for each auxiliary element. Instead, the noise correlation will be reduced in the software. As a result, only three of the five outputs of the array simulator are needed by the three-element SMI array processor. One of these three is the MAIN element output of the array simulator which is fed directly to the MAIN SIGNAL INPUT of the array processor. The other two signals required by the array processor may be chosen from the remaining four array simulator outputs. Each of the two chosen signals is split into two parts using a two-way power divider. One output of the power divider is fed to the correlator branch while the other is fed to the signal branch of an auxiliary branch. For example, if the AUX-1 SIGNAL output of the array simulator is chosen to be the signal received by the second auxiliary element of our array then the AUX-1 SIGNAL output is split into two parts with a twoway power divider. One output of the power divider is fed to the AUX-2 SIGNAL input of the array processor and the other output of the power divider is fed to the AUX-2 CORR input of the array processor. Care has been taken not to choose the AUX-1 CORR and AUX-2 CORR outputs of the array simulator to be received by the array processor auxiliary elements since to do so would create a scenario in which the auxiliary element signals have correlated noise components; contrary to an assumption made in the modified SMI algorithm derivation. To see this, recall that a single noise source (N2 of Figure 2.3) is used to generate the noise components of both the AUX-1 CORR and AUX-2 CORR outputs of the array simulator. 


\subsection{Sampling and Weighting the Main Chan- nel}

The fully adaptive SMI array requires that the main channel as well as the auxiliary channels be sampled and weighted. Since the original system was a sidelobe canceller, the main channel was not equipped with a VDM for sampling; nor does it have a VMOD for weighting the main element signal (see Figure 2.4). The problem of sampling the main channel is solved by noticing that, if the auxiliary element weights (VMOD-1 and VMOD2) are set to zero, the array output signal is equal to the main channel signal except for some attenuation and phase shift due to the real system components. Thus, the main channel is sampled by setting VMOD-1 and VMOD-2 to zero and sampling the output of VDM-3, the array output vector demodulator. The attenuation and phase shift will be offset by sample scaling and system calibration procedures that will be discussed in later sections of this chapter. In order to effectively weight the main channel, the weights computed by the SMI algorithm are always normalized so that the main element has unity weight. As a result, the main channel is weighted without a vector modulator.

\subsection{Scaling the Samples}

A significant difference between the SMI algorithm and the original Applebaum algorithm is that the SMI algorithm is open-loop whereas the Applebaum algorithm is closed-loop. In other words, the SMI algorithm derives weights using only the samples of the array element signals but the 
Applebaum algorithm uses the array output to adjust the array weights. The input to the SMI algorithm consists of a set of $\mathrm{K}$ snapshots. Each snapshot is a vector (of length 3 for a 3-element array or length 2 for a 2-element array) of samples resulting from a simultaneous sampling of the array elements. Since the SMI algorithm derivation assumes an ideal array, the snapshots presented to the algorithm must be identified with samples of the array element signals of an ideal array. To make this identification, the samples taken from the $A / D$ converter must be scaled in order to compensate for losses and phase shifts through the nonideal hardware components of our real array. Figure 3.1 compares the block diagrams of the ideal array and our experimental array. The $9 \mathrm{~dB}$ attenuators in the auxiliary channels represent the inherent loss through the vector modulators when the weights are set equal to unity. The first $6 \mathrm{~dB}$ attenuator at the output represents loss through the summer $\Sigma 1$ and the second represents loss through the 4-way power divider $\Delta 6$ of Figure 2.4. The $9 \mathrm{~dB}$ gain accounts for the fact that $\mathrm{A} 5$ and $\mathrm{A} 6$ are set $9 \mathrm{~dB}$ higher than $\mathrm{A} 1-\mathrm{A} 4$ in order to use the full dynamic range of the $A / D$ converter. The absolute settings of A1-A6 are not important since we are concerned only with relative gains. The large box of Figure 3.1 encloses an ideal array which "exists within" our real system. The dots denote the locations where samples from the ideal array exist; and such samples shall be called ideal samples. The samples which are available at the VDM outputs shall be called the real system samples. As seen in the figure the auxiliary element ideal samples are simply the corresponding real system samples attenuated by $9 \mathrm{~dB}$. The main element ideal samples are the real system output samples taken with the weights 
set to zero and boosted by $3 \mathrm{~dB}(=6+6-9)$. Also note that the ideal adapted array output samples which will be needed later to calculate output powers of the adapted array are the real system output samples taken with the weights set to their adapted values and boosted by $3 \mathrm{~dB}(=6+6-9)$. This scaling of samples is done in software as soon as the samples are retrieved from the A/D converter.

The scaling of samples described above represents a coarse amplitude adjustment in that, for example, the inherent loss through the first auxiliary element weight is not exactly 9dB. Furthermore, phase shifts through the real devices have not yet been offset. The further fine tuning which is required for the SMI algorithm to perform up to its abilities takes place in the weight calibration procedure which is discussed at the end of the chapter.

\subsection{The Modified SMI Algorithm}

This section describes the steps which are taken upon retrieving the scaled samples from the $A / D$ converter that results in a set of modified SMI weights to be implemented on the array. The strategy here will be to state the equation used to generate the weights, define the terms in this equation, and thoroughly discuss how these terms are computed.

In the Modified SMI algorithm a vector of complex weight estimates $\hat{W}_{K}$ based on $\mathrm{K}$ snapshots are computed using

$$
\hat{W}_{K}=\mu \hat{\Gamma}_{K}^{-1} \hat{S}
$$

where the notation ? will be used throughout this discussion to indicate esti- 

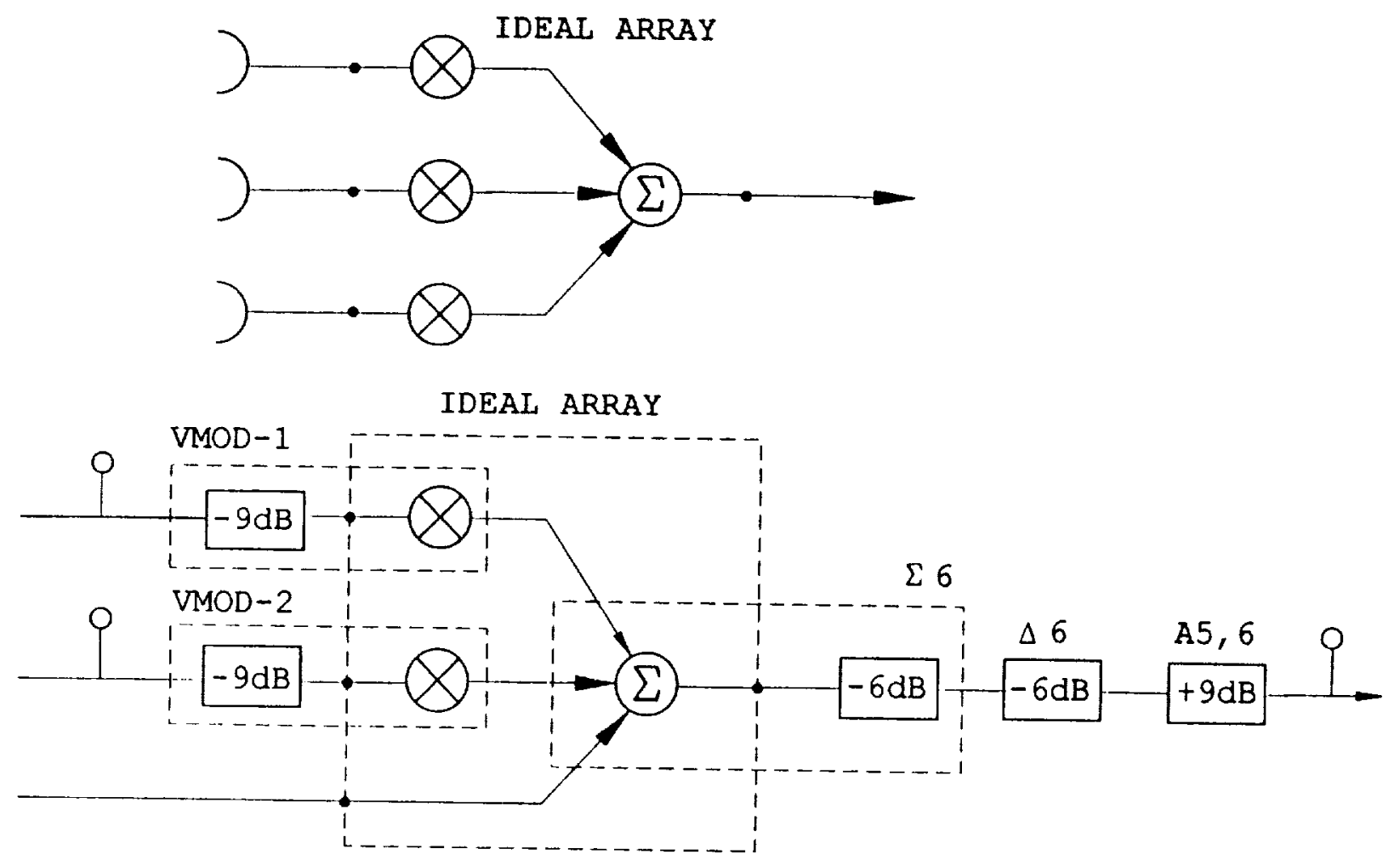

SIMPLE MODEL OF EXPERIMENTAL SYSTEM

Figure 3.1: Comparison of the ideal array and our experimental system. 
mate. For example, the algorithm yields an estimate based on $\mathrm{K}$ snapshots of an optimal weight vector. For our 3-element array, the weight vector is defined to be

$$
\hat{W}_{K}=\left[\begin{array}{c}
\hat{w}_{K 1} \\
\hat{w}_{K 2} \\
\hat{w}_{K 3}
\end{array}\right]=\left[\begin{array}{c}
\hat{w}_{K 1 I}-j \hat{w}_{K 1 Q} \\
\hat{w}_{K 2 I}-j \hat{w}_{K 2 Q} \\
\hat{w}_{K 3 I}-j \hat{w}_{K 3 Q}
\end{array}\right]
$$

where $\hat{w}_{K 1}$ is the estimated complex weight for the first auxiliary element, $\hat{w}_{K 2}$ is the estimated complex weight for the second auxiliary element, and $\hat{w}_{K 3}$ is the estimated complex main channel weight. In order to apply a complex weight in our real array system each VMOD is provided the inphase and quadrature components of the proper complex weight. Equation (3.2) defines the in-phase and quadrature components (subscripts $I$ and $Q$, respectively) of the complex weights. The minus sign is included in the definition since a VMOD input signal is split (within the VMOD) into two components such that the quadrature-weighted component lags the in-phase-weighted component by 90 degrees.

In Equation (3.1), $\mu$ is an arbitrary complex scalar which is chosen here so that $\hat{w}_{K 3}=1+j 0$. This normalization is necessary for our array system since the main channel is not equipped with its own VMOD. $\hat{\Gamma}_{K}$ is the $(3 \times 3)$ modified sample (estimated) covariance matrix given by

$$
\hat{\Gamma}_{K}=\hat{\Phi}_{K}-F \lambda_{\min }\left(\hat{\Phi}_{K}\right) I
$$

and $\hat{S}$ is the steering vector. The method for generating a steering vector is presented at the end of this chapter.

In Equation (3.3), the standard sample covariance matrix $\hat{\Phi}_{K}$ based on 
K complex signal snapshots $\left\{X_{k}\right\}_{k=1}^{K}$ is the block average

$$
\hat{\Phi}_{K}=\frac{1}{K} \sum_{k=1}^{K} X_{k} X_{k}^{H}
$$

where ${ }^{H}$ indicates Hermitian transpose. Also in Equation (3.3), $F$ is the fraction between 0 and 1 which is chosen to achieve a desired level of interference suppression, $\lambda_{\min }\left(\hat{\Phi}_{K}\right)$ is the minimum of the 3 real positive eigenvalues of the positive definite Hermitian matrix $\hat{\Phi}_{K}$, and $I$ is the $(3 \times 3)$ identity matrix.

The procedure for computing the array weights given $\mathrm{K}$ complex $(3 \times 1)$ signal snapshots is clear. First, the standard sample covariance matrix $\hat{\boldsymbol{\Phi}}_{K}$ is computed as in Equation (3.4) and its minimum eigenvalue is found. These quantities are used in Equation (3.3) along with the chosen value of F to yield the modified sample covariance matrix $\hat{\Gamma}_{K}$. Next, $\hat{\Gamma}_{K}$ and the approximate steering vector $\hat{S}$ are substituted into Equation (3.1) to yield the complex weights $\hat{W}_{K}$. Remember that $\mu$ is chosen to make $\hat{w}_{K 3}=1+j 0$. Finally, the I and $\mathrm{Q}$ components of each complex weight are found from Equation (3.2) and are provided to the VMODs in analog. The next task is to describe how the complex signal snapshots $X_{k}$ are generated.

Let $x_{k 1}, x_{k 2}, x_{k 3}$, be the scaled (see Section 3.3) complex samples of the signals received at the first auxiliary, second auxiliary, and main channel at time index k. Recall, for example, that $x_{k 3}$ is a scaled version of a sample taken through VDM-3 while the auxiliary element weights are set to zero. The $\mathrm{k}^{\text {th }}$ signal snapshot $X_{k}$ is a vector of these samples given by

$$
X_{k}=\left[\begin{array}{l}
x_{k 1} \\
x_{k 2} \\
x_{k 3}
\end{array}\right]=\left[\begin{array}{l}
x_{k 1 I}+j x_{k 1 Q} \\
x_{k 2 I}+j x_{k 2 Q} \\
x_{k 3 I}+j x_{k 3 Q}
\end{array}\right] .
$$


In the communication signal environment, the $k^{\text {th }}$ sample of the $n^{\text {th }}$ element will contain desired, interference, and noise components, and thus can be written

$$
x_{k n}=x_{k n D}+\sum_{m=1}^{M} x_{k n I m}+x_{k n \eta}
$$

for $\mathrm{M}$ interference signals. Using (3.6) in (3.5), the $k^{\text {th }}$ snapshot vector is written in terms of its signal component vectors

$$
X_{k}=X_{k D}+\sum_{m=1}^{M} X_{k I m}+X_{k \eta}
$$

The SMI algorithm uses $K$ such snapshots to form the covariance matrix estimate $\hat{\Phi}_{K}$ which can be written using (3.7) in (3.4) as

$$
\begin{aligned}
\hat{\Phi}_{K} & =\frac{1}{K} \sum_{k=1}^{K}\left(X_{k D}+\sum_{m=1}^{M} X_{k I m}+X_{k \eta}\right)\left(X_{k D}+\sum_{m=1}^{M} X_{k I m}+X_{k \eta}\right)^{H} \\
& =\frac{1}{K} \sum_{k=1}^{K}\left(X_{k D} X_{k D}^{H}+\sum_{m=1}^{M} X_{k I m} X_{k I m}^{H}+X_{k \eta} X_{k \eta}^{H}\right) \\
& +\frac{1}{K} \sum_{k=1}^{K}\left(X_{k \eta} \sum_{m=1}^{M} X_{k I m}^{H}+\sum_{m=1}^{M} X_{k I m} X_{k \eta}^{H}\right) \\
& +\frac{1}{K} \sum_{k=1}^{K}\left(X_{k D} X_{k \eta}^{H}+X_{k \eta} X_{k D}^{H}\right) \\
& +\frac{1}{K} \sum_{k=1}^{K}\left(X_{k D} \sum_{m=1}^{M} X_{k I m}^{H}+\sum_{m=1}^{M} X_{k I m} X_{k D}^{H}\right) \\
& +\frac{1}{K} \sum_{k=1}^{K} \sum_{\substack{m_{1}=1 \\
m_{1} \neq m_{2}}}^{M} \sum_{m_{2}=1}^{M} X_{k I m_{1}} X_{k I m_{2}}^{H} .
\end{aligned}
$$

The first line of the final expression in (3.8) approaches the true covariance matrix (which we are estimating) whereas the last four lines approach zero as $K \rightarrow \infty$. 
When signals received from geosynchronous satellites are used as input to the array processor, a snapshot is formed as a vector of signal samples generated by simultaneously sampling the vector demodulator VDM outputs of the array processor (see Figure 2.4) and applying the calibration factors (see Sections 3.3 and 3.6). However, when the bench-generated signals (i.e. the outputs of the array simulator) are used as inputs to the array processor, care must be taken in forming a set of $K$ snapshots $\left\{X_{k}\right\}_{k=1}^{K}$ so as not to take advantage of the special pulse-modulated signal scheme employed by our experimental system. Recall that the desired and interference signals are separated in time (see Figure 2.5) so that their powers can be calculated and our system performance evaluated. Thus, if (when using bench-generated signals) a snapshot is formed by simply combining the samples taken at a single instant of time then no single snapshot would contain both desired and interference signal components. As a result, the last two lines of (3.8) would not be present in the resulting covariance matrix estimate. Although this lack of desired-interference crossterms would not change the asymptotic value of the covariance estimate it would give an unrealistic (overly-optimistic) picture of the performance of the array based on a finite number of samples. To remedy this situation, a single snapshot $X_{T}$ containing all signal components is "built" by simply summing three phase-shifted single-component snapshots; making sure that one of the addends comes from the desired signal portion of the pulse repetition period (call this snapshot $X_{D}$ ), another $\left(X_{I_{1}}\right)$ from the first interference signal portion, and the third $\left(X_{I 2}\right)$ from the second interference portion (see Figure 2.5). An addend from the noise-only portion of the pulse repetition period 
is not necessary since noise is already present in the other three addends. Thus, a typical bench-generated snapshot is formed as

$$
X_{T}=e^{j \phi_{1}} X_{D}+e^{j \phi_{2}} X_{I 1}+e^{j \phi_{3}} X_{I 2} .
$$

The phase angles $\phi_{1}, \phi_{2}$, and $\phi_{3}$ are i.i.d. uniform random variables on $[0,2 \pi]$. The random phases are needed to decorrelate the bench-generated desired and interference signals. This apparent correlation between received signals was a byproduct of using a single $69 \mathrm{MHz}$ oscillator to generate the carrier for the desired and interference pulse signals. The summing procedure (3.9) for forming bench-generated snapshots was used in [6] in order to study the performance of the system as a function of the number of snapshots.

This report is specifically concerned with the steady state performance of the modified SMI system. Thus, it is to our advantage here to omit the last two lines of (3.8) from the covariance estimate by not using the snapshot summing procedure of (3.9). In other words, in this report, a snapshot is formed as a vector of (calibrated) samples which were taken at a single instant of time. In doing so we shorten the sample-taking process.

The above presentation of the SMI algorithm was formulated in terms of a three-element array. The experimental array is also capable of implementing a two-element array by simply ignoring one of the two auxiliary elements. For this two-element configuration the algorithm must be adjusted accordingly. In particular, the estimated weight vector $\hat{W}_{K}$ becomes length 2 , with the first entry being the auxiliary element weight and the second entry being the main element weight. As before, the weight vector is 
normalized so that the main element weight $\left(\right.$ now $\left.\hat{w}_{K 2}\right)$ is unity. Similarly, the snapshot vector becomes length 2 with the auxiliary element sample occupying the first entry and the main element sample occupying the second entry.

It may have occurred to the reader that the calculation of input and output signal and noise powers must be thought out carefully so that the performance results obtained here relate directly to results found in the literature. The power calculation procedure is discussed next, followed by the topics of system calibration and steering vector generation to conclude the chapter.

\subsection{Power Calculations}

In order to quantitatively examine the steady-state performance of the experimental modified SMI array it is necessary to calculate (from a set of samples) the desired and interference signal powers and the noise power in the adapted array output signal and the two or three input signals (depending on whether a two- or three-element array is being implemented). Using these power measurements, quantities such as interference suppression (IS) and output signal-to-noise ratio (SNR) can be found and compared with those of theory. Comparison of theoretical and experimental system results for several steady-state experiments appear in the next chapter. This section explains the procedure for calculating the various signal powers, IS, SNR, etc.

Figure 3.2 shows the I and $Q$ outputs of one vector demodulator (VDM). 
The D pulses are the desired signal, and $I 1$ and $I 2$ are the interfering signals. Due to the pulse modulation, the desired signal amplitudes are calculated from the sampled data over time interval $\tau_{1}$, and the interfering signal amplitudes are calculated from the samples over $\tau_{2}$ and $\tau_{3}$. Samples over interval $\tau_{4}$ are used to compute the noise power in each channel and, in addition, are used to calculate and correct for any DC offset voltages due to the detector amplifiers (A1-A6 of Figure 2.4). Even though these offset voltages have been nulled by adjustments at the amplifiers themselves, this correction is done in software as a precaution against any drift that may occur during the course of an experiment. Left uncorrected, such offset voltages would cause errors in the estimated covariance matrix and thus degrade adaptive array performance.

The signal level and noise power calculations are based on finite numbers of samples of a noisy signal and thus are estimates of the actual levels. In the experiments to be conducted, the suppressed interference components in the adapted array output signal will be as much as $30 \mathrm{~dB}$ below the noise level, making their levels very difficult to estimate accurately. The number of samples required for accurate estimates of the output interference signal levels (as well as all other signal levels) is reduced by simply turning off the noise generators while samples for signal power calculation are being taken. Of course, the noise generators are "on" when samples for weight and noise power estimation are taken..

Let $x_{k J I}$ and $x_{k J Q}$ be the $k^{\text {th }}$ scaled I and Q samples of element $J$ for $J=1,2$, and 3 , as defined in Section 3.4. These samples are not the very same samples used to form the snapshots needed for weight computation 


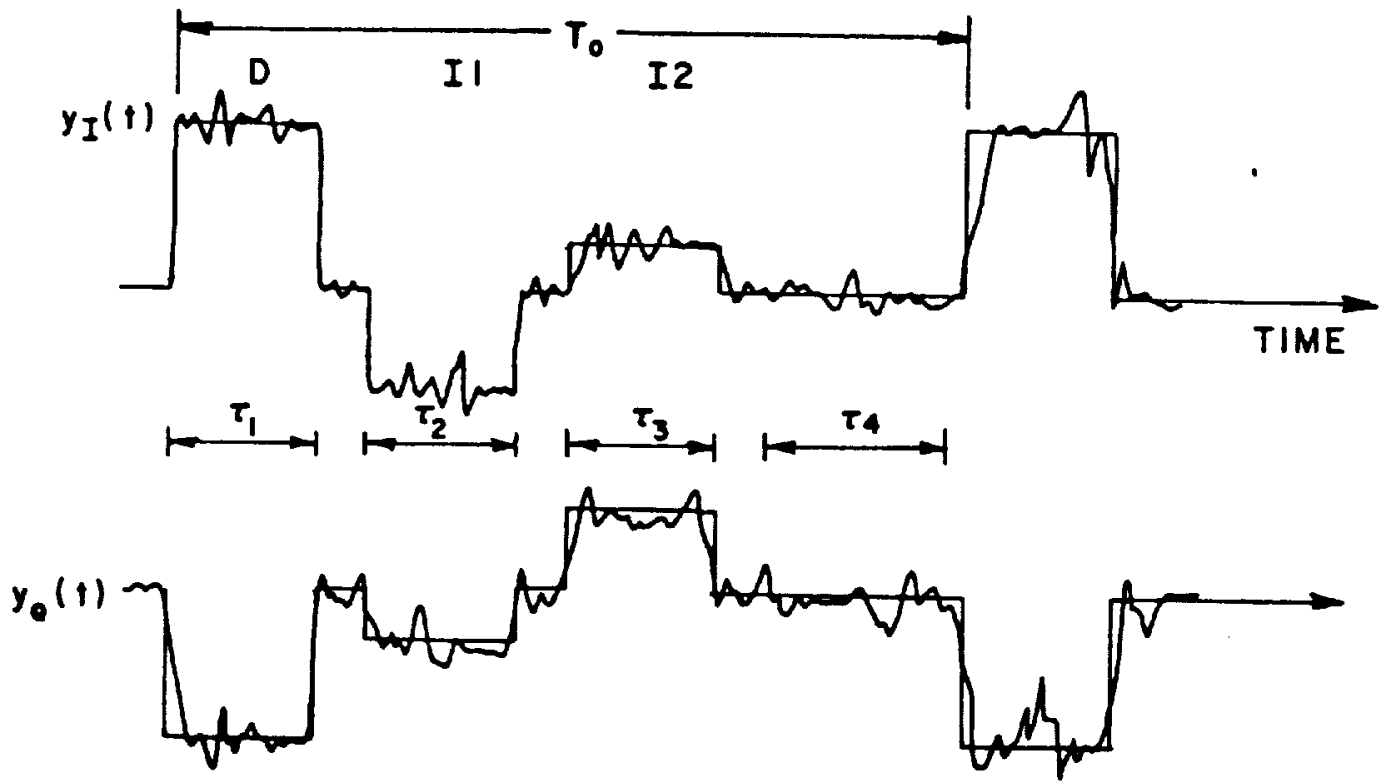

Figure 3.2: Typical I and Q VDM outputs.

but instead are different samples of the same signals. Furthermore, newly define $x_{k 4 I}$ and $x_{k 4 Q}$ to be the $k^{\text {th }}$ scaled I and Q samples of the adapted array output signal. Suppose that this new data has been taken for $k$ ranging from 1 to $K 1$ in each of the 4 time intervals $\tau_{i}$ of Figure 3.2. Then compute

$$
\begin{aligned}
& \hat{O}_{J P}=\frac{1}{K 1} \sum_{k=1}^{K 1} x_{k J P} \text { on } \tau_{4} \\
& \hat{D}_{J P}=\frac{1}{K 1} \sum_{k=1}^{K 1} x_{k J P}-\hat{O}_{J P} \text { on } \tau_{1}
\end{aligned}
$$




$$
\begin{aligned}
& \hat{I}_{J P}=\frac{1}{K 1} \sum_{k=1}^{K 1} x_{k J P}-\hat{O}_{J P} \text { on } \tau_{2} \\
& \hat{I}_{J P}=\frac{1}{K 1} \sum_{k=1}^{K 1} x_{k J P}-\hat{O}_{J P} \text { on } \tau_{3} \\
& \hat{\sigma}_{J P}^{2}=\frac{1}{K 1} \sum_{k=1}^{K 1}\left(x_{k J P}-\hat{O}_{J P}\right)^{2} \text { on } \tau_{4}
\end{aligned}
$$

for $J \in\{1,2,3,4\}$ and $P \in\{I, Q\} . \hat{O}_{J I}$ and $\hat{O}_{J Q}$ are the estimates of the offsets in the $\mathrm{I}$ and $\mathrm{Q}$ components of array signal $J . \hat{D}_{J I}$ and $\hat{D}_{J Q}$ are the estimates of the $I$ and $Q$ component amplitudes of the desired signal component in array signal $J . \hat{I}_{J I}, \hat{I}_{J Q}, \hat{I}_{J I}$, and $\hat{I}_{J Q}$ are analogous parameters for the first and second interference signals, respectively. Recall that the samples for the estimates of (3.10)-(3.13) are taken with the noise generators turned off. $\hat{\sigma}_{J I}^{2}$ and $\hat{\sigma}_{J Q}^{2}$ are the noise power estimates in the I and $Q$ channels of array signal J.

From these equations we see that the signal amplitude calculations for the desired and interference signals are obtained by sample averaging over the particular portion of the pulse repetition period corresponding to the signal under measurement. The variances of these signal level estimates will depend on the average noise power $\sigma^{2}$ present in the particular array signal being averaged and on the number of samples $K 1$ in the average. Specifically, the averages (3.10)-(3.13) are efficient maximum likelihood estimates of the $I$ and $Q$ offsets and signal amplitudes. The variance of the offset estimate of (3.10) is $\sigma^{2} / K 1$ whereas the variance of the signal level estimates of (3.11)-(3.13) is $2 \sigma^{2} / K 1$. The noise power estimate of (3.14) is asymptotically unbiased [8]. 
From the I and $Q$ signal amplitude and noise power estimates in array signal $\mathrm{J}$, the average power of the desired, interference, and noise components of complex array signal $\mathrm{J}$ are computed as follows:

$$
\begin{aligned}
& \hat{P}_{D J}=\hat{D}_{J I}^{2}+\hat{D}_{J Q}^{2} \\
& \hat{P}_{I 1 J}=\hat{I}_{J I}^{2}+\hat{I 1}_{J Q}^{2} \\
& \hat{P}_{I 2 J}=\hat{I}_{J I}^{2}+\hat{I}_{J Q}^{2} \\
& \hat{P}_{\eta J}=3\left(\hat{\sigma}_{J I}^{2}+\hat{\sigma}_{J Q}^{2}\right) .
\end{aligned}
$$

The factor of 3 in (3.18) is included to account for the fact that each signal snapshot used in the weight estimate contains a noise component whereas the desired signal is present in just $1 / 3$ of these snapshots as are the first and second interference signals. Because the $I$ and $Q$ components of narrowband noise are uncorrelated [9], the total average noise power in a particular channel is the sum of the noise powers at the $I$ and $Q$ outputs of that VDM.

Once the power of each signal component (desired, interference, noise) in each array signal (first auxiliary, second auxiliary, main channel, adapted array output) has been computed any interesting performance measures can be easily found. For example, the output signal-to-noise ratio (SNR), first interference suppression (IS1), second interference suppression (IS2), and total interference suppression (IST) are just

$$
\begin{aligned}
S N R & =\frac{P_{D 4}}{P_{\eta 4}} \\
I S 1 & =\frac{P_{I 14}}{P_{I 13}}
\end{aligned}
$$




$$
\begin{aligned}
I S 2 & =\frac{P_{I 24}}{P_{I 23}} \\
I S T & =\frac{P_{I 14}+P_{I 24}}{P_{I 13}+P_{I 23}} .
\end{aligned}
$$

In Chapter 4, these performance measures will be used to compare the performance of the experimental system with that of an ideal modified SMI array operating in the same signal environment. Before the experimental array can perform up to its capabilities, however, the system must be calibrated. System calibration is discussed in the next section.

\subsection{System Calibration}

The experimental system uses a software calibration procedure to correct for non-ideal characteristics (i.e. losses and phase shifts) of the real system components. Recall that the sample scaling of Section 3.3 represented a coarse adjustment of sample amplitudes. Phase corrections and further amplitude corrections to the samples are necessary to ensure proper array performance.

The calibration procedure used here adapts the weights for the simple scenario of a 2-element array receiving only a single interference signal (no noise, no desired signal). Since the scaled samples are not ideal, the estimated weights will not be ideal. However, ideally we know that the array should suppress the interference at the array output beneath measureable levels. By scaling the amplitude and shifting the phase of the adapted auxiliary element weight, an ideal weight which completely suppresses the interference is found. The amplitude scale factor $A$ and phase 
shift $\phi$ required to transform the adapted auxiliary element weight to the ideal weight can be represented by the complex number $c=A e^{j \phi}$. By applying the "fine-tuning" sample adjustment factor $1 / c^{*}$ to the scaled auxiliary element samples in subsequent experiments the resulting adapted auxiliary element weight is made equal to the ideal weight. To see this, suppose the fine-tuning adjustment factor for the first auxiliary element samples were known; call it $1 / c^{*}$. Then the $k^{\text {th }}$ fine-tuned snapshot $X_{k F T}$ may be written in terms of the $k^{\text {th }}$ coarse-adjusted snapshot $X_{k C A}$ (see Section 3.3) as

$$
\begin{aligned}
X_{k F T} & =\left[\begin{array}{cc}
\frac{1}{c^{*}} & 0 \\
0 & 1
\end{array}\right] X_{k C A} \\
& \equiv C X_{k C A} .
\end{aligned}
$$

As a reminder, we are considering the two-element array in which the first auxiliary element sample occupies the first element of the snapshots and the main element sample occupies the second element of the snapshots. Thus, (3.23) implies that we are calibrating the first auxiliary element samples with respect to the main element samples. The estimated covariance matrix $\hat{\Phi}_{F T}$ based on $\mathrm{K}$ of the fine-tuned snapshots may be written in terms of the corresponding covariance matrix $\hat{\Phi}_{C A}$ based on the coarse-adjusted snapshots. Using (3.24) in (3.4), we have

$$
\begin{aligned}
\hat{\Phi}_{F T} & =\frac{1}{K} \sum_{k=1}^{K} X_{k F T} X_{k F T}^{H} \\
& =\frac{1}{K} \sum_{k=1}^{K}\left[C X_{k C A}\right]\left[C X_{k C A}\right]^{H} \\
& =\frac{1}{K} \sum_{k=1}^{K} C X_{k C A} X_{k C A}^{H} C^{H}
\end{aligned}
$$




$$
\begin{aligned}
& =C\left[\frac{1}{K} \sum_{k=1}^{K} X_{k C A} X_{k C A}^{H}\right] C^{H} \\
& =C \hat{\Phi}_{C A} C^{H} .
\end{aligned}
$$

Using (3.25) in (3.3) with $F=0$ (standard SMI) and substituting the result in (3.1), we may express the standard SMI weights $\hat{W}_{F T}$ based on K fine-tuned snapshots as

$$
\begin{aligned}
\hat{W}_{F T} & =\mu \hat{\Phi}_{F T}^{-1} S \\
& =\mu\left[C \hat{\Phi}_{C A} C^{H}\right]^{-1} S \\
& =\mu\left(C^{H}\right)^{-1} \hat{\Phi}_{C A}^{-1} C^{-1} S \\
& =\mu\left[\begin{array}{ll}
c & 0 \\
0 & 1
\end{array}\right] \hat{\Phi}_{C A}^{-1}\left[\begin{array}{cc}
c * & 0 \\
0 & 1
\end{array}\right] S .
\end{aligned}
$$

Let $\phi_{i j}^{-1}$ be the $i j^{\text {th }}$ element of $\hat{\Phi}_{C A}^{-1}$. Also since the desired signal is not present in this scenario, we are free to choose $S=\left[\begin{array}{ll}0 & 1\end{array}\right]^{T}$. When we make these substitutions in (3.26) and evaluate, we have

$$
\begin{aligned}
\hat{W}_{F T} & =\mu\left[\begin{array}{ll}
c & 0 \\
0 & 1
\end{array}\right]\left[\begin{array}{ll}
\phi_{11}^{-1} & \phi_{12}^{-1} \\
\phi_{21}^{-1} & \phi_{22}^{-1}
\end{array}\right]\left[\begin{array}{cc}
c * & 0 \\
0 & 1
\end{array}\right]\left[\begin{array}{l}
0 \\
1
\end{array}\right] \\
& =\mu\left[\begin{array}{cc}
|c|^{2} \phi_{11}^{-1} & c \phi_{12}^{-1} \\
c^{*} \phi_{21}^{-1} & \phi_{22}^{-1}
\end{array}\right]\left[\begin{array}{l}
0 \\
1
\end{array}\right] \\
& =\mu\left[\begin{array}{c}
c \phi_{12}^{-1} \\
\phi_{22}^{-1}
\end{array}\right] \\
& =\left[\begin{array}{c}
c \hat{w}_{1 C A} \\
\hat{w}_{2 C A}
\end{array}\right]
\end{aligned}
$$

where $\hat{w}_{1 C A}$, and $\hat{w}_{2 C A}$ are the auxiliary and main element weights based on the corresponding course-adjusted (not fine-tuned) snapshots. In words, (3.23) and (3.27) state that the effect of fine-tuning the auxiliary element samples with the factor $1 / c *$ is to change the resulting auxiliary element 
weight by the factor $c$. Recall that this result assumes a particular scenario and $S=\left[\begin{array}{ll}0 & 1\end{array}\right]^{T}$. However, the sample scale factor $1 / c^{*}$ which depends on cable lengths and real component characteristics should be independent of signal scenario and choice of steering vector.

Our goal is to determine the scale factor $c$. To do this, the singleinterference scenario described above is set up and the uncalibrated (coarseadjusted but not fine-tuned) system is used to estimate a coarse-adjusted auxiliary element weight $\hat{w}_{1 C A}$ which does not yield a completely suppressed interference signal at the array output. By stepping the magnitude and phase of the auxiliary element weight through a range of values, we determine the ideal fine-tuned weight $\hat{w}_{1 F T}$ which causes the output interference signal to be completely suppressed. We then calculate $c=\hat{w}_{1 F T} / \hat{w}_{1 C A}$ (using the result in (3.27)) and apply the fine-tuning factor $1 / c^{*}$ (see (3.23)) to the first auxiliary element samples in subsequent experiments. The analogous procedure is followed to fine tune the second auxiliary element samples.

This calibration process may be fully implemented in software by using the output interference power measurement capability described in the previous section as follows:

\section{Calibration Procedure}

I Set up the scenario

A. 2-element array (main and first auxiliary).

B. Only first interference signal, no desired signal, no added noise.

C. Set attenuator $a_{4}$ of Figure 2.3 so that a significant amount of interference is injected into the main channel to be 
cancelled with the interference in the first auxiliary.

II Adapt the weight

A. Take samples for snapshot formation (enough for steady state estimation).

B. Estimate the first auxiliary element weight $w$. Note: Since the weight is uncalibrated, it needs to be scaled in phase and magnitude.

III Find phase and magnitude scale factors $\left(\phi_{o}\right.$ and $\left.M_{o}\right)$.

A. Let $M_{o}=1$ and $\phi_{o}=0$.

B. Step the phase of the weight $w M_{o} e^{j \phi_{o}}$ through a range of values. At each step (varying $\phi$ ):

i. Apply the phase-adjusted weight $e^{j \phi} w M_{o} e^{j \phi_{o}}$.

ii. Calculate and save the interference power at the array output.

C. Choose the phase $\phi$ that yielded the minimum output interference power, call it $\phi_{a}$.

D. Set $\phi_{0}=\phi_{0}+\phi_{a}$.

E. Scale the magnitude of the weight $w M_{\circ} e^{j \phi_{o}}$ through a range of values. At each step (varying $M$ ):

i. Apply the magnitude-scaled weight $M w M_{\mathrm{o}} e^{j \phi_{0}}$.

ii. Calculate and save the interference power at the array output.

F. Choose the magnitude scale factor $M$ that yielded the minimum output interference power, call it $M_{a}$.

G. Set $M_{o}=M_{o} M_{a}$.

H. Repeat IIIB.-IIIG. until convergence of $M_{0}$ and $\phi_{0}$.

IV In all subsequent experiments modify the first auxiliary element (scaled) samples by the factor $e^{j \phi_{0}} / M_{0}$. 
V Repeat I-IV using the second auxiliary element instead of the first in order to fine tune the second auxiliary element (scaled) samples.

The above procedure has been made iterative because the VMOD's do not give us completely independent control of the weight magnitude and phase. Let us now turn to the problem of generating a steering vector.

\subsection{Estimation of the Steering Vector}

The steering vector contains the information that allows the SMI array to distinguish the desired signal from the interfering signals. In order to estimate the steering vector for a given bench-generated signal scenario we begin by turning off the interference signals and noise generators so that only the desired signal and negligible system noise are present in the received signals. We then take snapshots and form the estimate

$$
\hat{S}=\frac{1}{K} \sum_{k=1}^{K} x_{k 1}^{*} X_{k}
$$

where $X_{k}$ is the $k^{\text {th }}$ snapshot and $x_{k 1}$ is the first element of $X_{k}$. We note in passing that (3.28) is simply the first column of the standard sample covariance matrix (3.4). Once the steering vector estimate is stored in memory, the interference signals and noise generators are restored to the desired levels. This method of steering vector generation will be used in later experiments. Throughout this report, however, the main antenna is of relatively large gain compared to the auxiliary elements. This assumption 
allows us to approximate the steering vector as

$$
\hat{S}=\left[\begin{array}{lll}
0 & 0 & 1
\end{array}\right]^{T}
$$

where ${ }^{T}$ denotes transpose and the third element of $\hat{S}$ corresponds to the main element. The subject of generating a steering vector when the received signals are from geosynchronous satellites is not addressed here. Now that the experimental modified SMI system has been described and all relevent procedures have been discussed, it is time to describe its performance through experimentation. 


\section{Chapter 4}

\section{Experimental Results}

The experiments test the steady state performance of the modified SMI array system for various input signal scenarios. By steady state performance we mean that performance achieved by using many snapshots in the weight estimation. The performance is studied using a step-by-step approach. First, the system is tested using standard SMI (i.e. with $F=0$ ). These tests establish the invariance of the calibration factors to changes in signal scenario, compare the experimental results with theory, and point out a lack of suppression of weak interference. The modified SMI algorithm is then used to increase the suppression of weak interference while maintaining a strong desired signal. In all experiments, the noise power in each element of the array is (approximately) the same and the desired signal, when present, is incident from broadside and present only in the main channel. The procedure used for conducting an experiment is

1. Set the attenuators of the array simulator so as to present certain desired and interference signal levels to the array processor. 
2. Turn off the noise generators.

3. Set the weights to zero.

4. Estimate the desired and interference signal levels using (3.15)-(3.17) (for $\mathrm{J}=1,2$, and 3 )

5. Set the noise generators to the desired levels.

6. Estimate the noise power in each array element using (3.18) (for $\mathrm{J}=$ 1,2 , and 3).

7. Collect samples with which to form snapshots (making sure that enough samples are taken to yield near steady state weights).

8. Estimate the modified SMI array weights.

9. Apply the estimated weights.

10. Estimate noise power in the array output using (3.18) with $\mathrm{J}=4$.

11. Turn off noise generators.

12. Estimate the desired and interference signal powers in the array output $(\mathrm{J}=4)$ using $(3.15)-(3.17)$.

13. Calculate performance parameters such as SNR and IS1 of (3.19) and $(3.20)$. 


\subsection{Experiment 1: Two elements, one inter- ference, standard SMI, vary interference power}

In the first experiment, each auxiliary channel is tested separately by using the two-element (main and a single auxiliary) capability of the experimental system and standard SMI $(F=0)$. No desired signal is present. A single interference of varying power is incident on the array from a fixed direction. The direction of arrival ( $52^{\circ}$ off broadside in this case) is unimportant since any element pattern considerations are absorbed into the received signal powers. INR(aux)-INR(main) is fixed at $5.5 \mathrm{~dB}$. Figure 4.1 shows the interference suppression versus INR(main) for experiment and theory. One of the experimental curves corresponds to the two-element array consisting of the main and first auxiliary elements whereas the other corresponds to the main and second auxiliary element combination. A good agreement between theory and experiment is observed. In the linear region of the plot, a $5 \mathrm{~dB}$ increase in interference power leads to a $10 \mathrm{~dB}$ increase in its suppression. In the region $\operatorname{INR}($ main $)=10 \sim 15 \mathrm{~dB}$ the small output interference power becomes difficult to measure even with the noise generators turned off, due to ever-present residual noise; the main sources of which are the detector amplifiers $\mathrm{A} 5$ and $\mathrm{A6}$. Note that for weak interference (INR(main) $<-5 \mathrm{~dB}$ ), standard SMI yields less than $7 \mathrm{~dB}$ of interference suppression. 
aux1 and main, latt $=5.5 \mathrm{~dB}$

aux2 and main, latt $=5.5 \mathrm{~dB}$

theory for 2-element array, latt $=5.5 \mathrm{~dB}$

One interference, no desired, main and one aux.

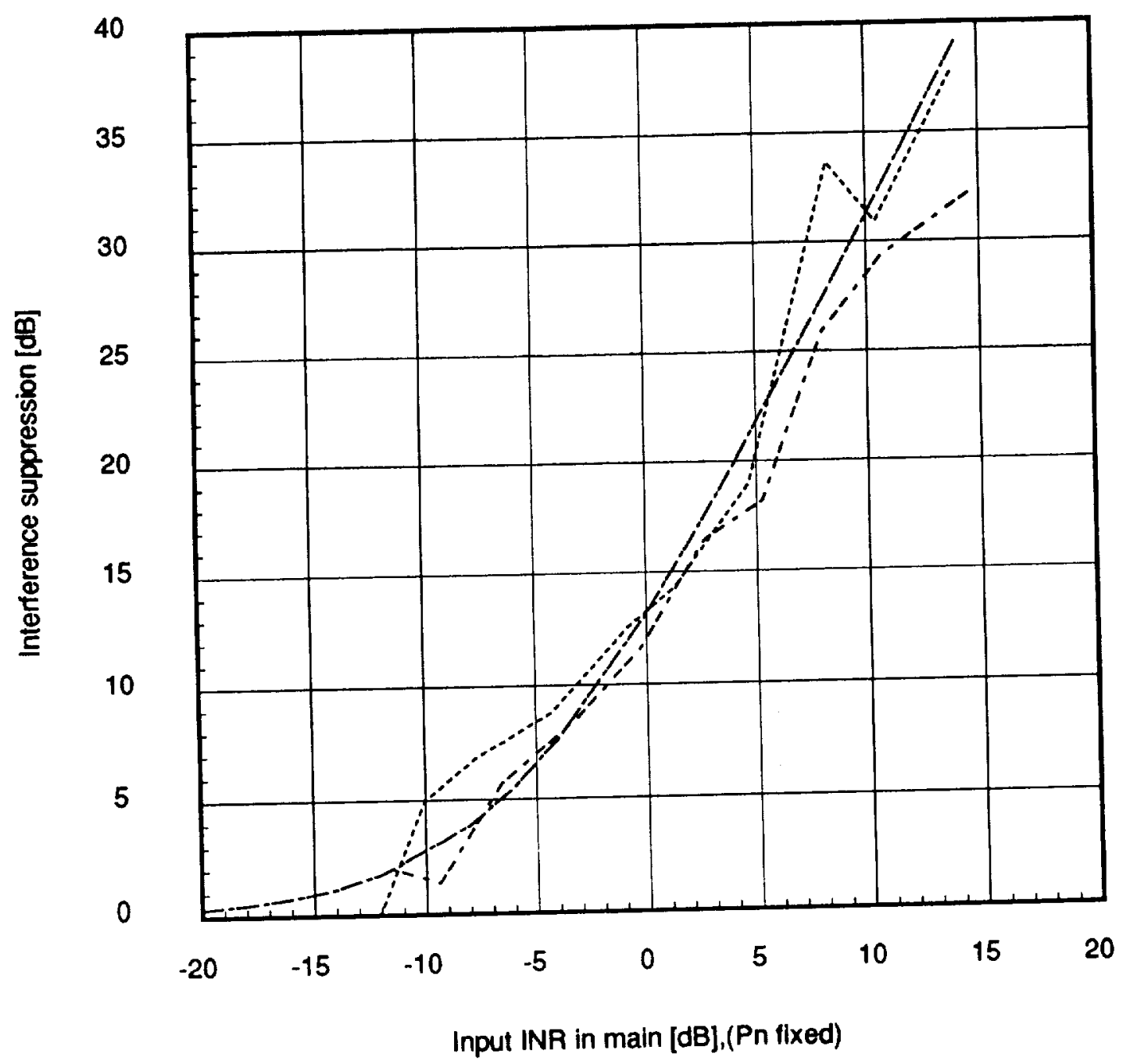

Figure 4.1: Experiment 1: Suppression of a single interference of varying power with a two-element array. 


\subsection{Experiment 2: Three elements, one in- terference, desired signal, standard SMI, vary interference arrival angle}

Rather than varying the power level of the single interference we vary the direction of arrival (DOA) in Experiment 2. This time, standard SMI and all three elements are used and $\mathrm{SNR}($ main $)=16 \mathrm{~dB}$. The desired signal level in the auxiliary elements is negligible. The purpose here is to test the phase shifters of the array simulator and the phase-shifting ability of the vector modulators (weights). In addition, the invariance of the calibration factors to different scenarios is tested. The fixed input interference signal levels appear in Table 4.2. Figure 4.2 shows the results. One set of curves (theory and experiment) corresponds to the first interference signal whereas the other pair corresponds to the second interference signal. Five data points were used to form each experimental curve. There is reasonable agreement between theory and experiment since only one data point is further than $2 \mathrm{~dB}$ from its theoretical value. As expected, interference suppression is theoretically and experimentally independent of the arrival angle (as long as desired and interference signals arrive from different directions). Also note that the level of interference suppression provided by the standard SMI algorithm for this particular scenario is about $7.5 \mathrm{~dB}$. This may not be enough in some applications. 
int.\#1, theory

int.\#1, exper.

int.\#2, theory

int.\#2, exper.

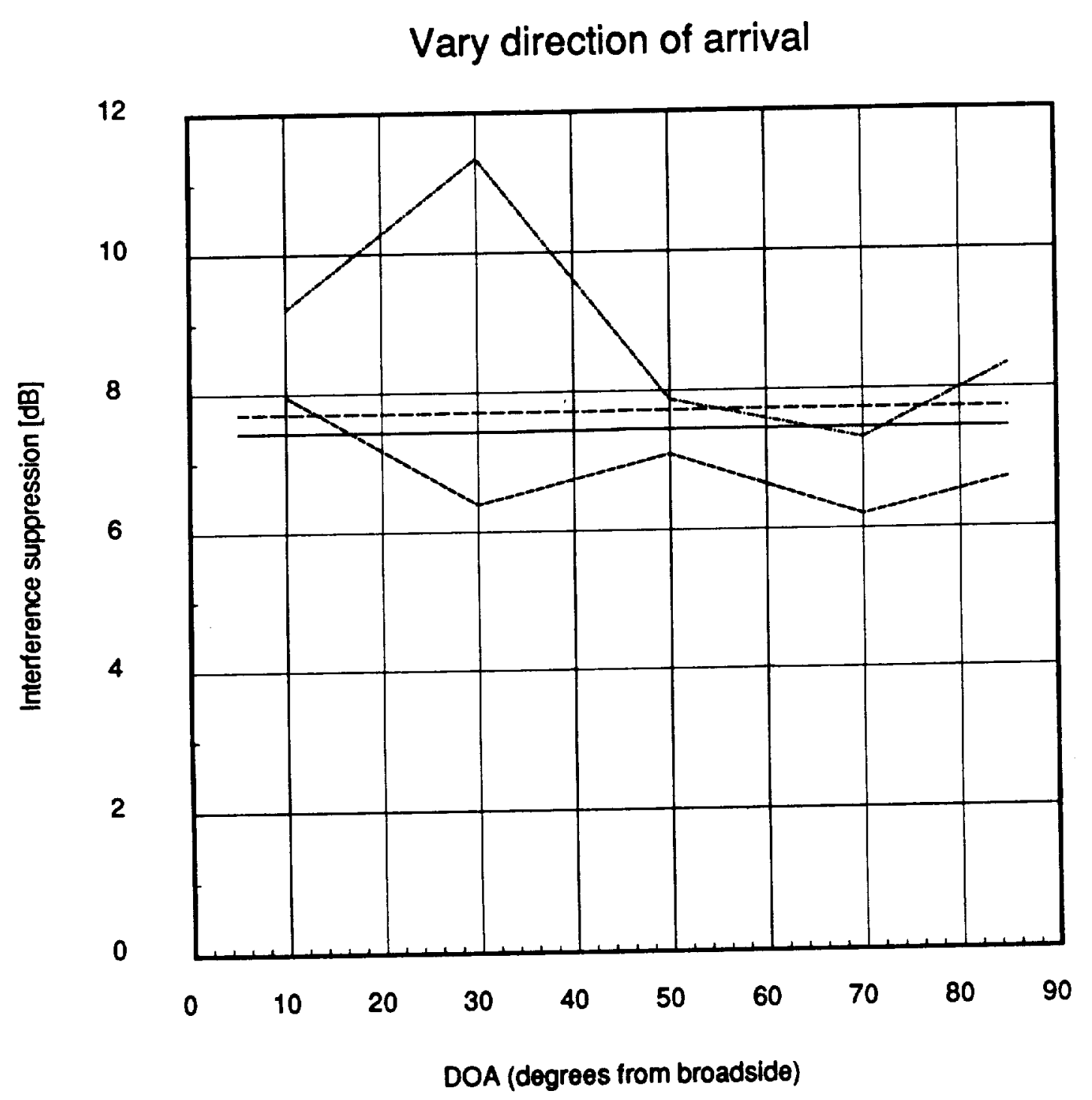

Figure 4.2: Experiment 2: Suppression of a single interference of varying arrival angle with a 3 -element array and $\mathrm{SNR}($ main $)=16 \mathrm{~dB}$. 


\begin{tabular}{||l||r|r|r||}
\hline \hline & INR(main) & INR(aux1) & INR(aux2) \\
\hline \hline Int. 1 only & -3.78 & 1.21 & -16.84 \\
\hline Int. 2 only & -4.67 & -16.48 & 1.47 \\
\hline \hline
\end{tabular}

Table 4.1: Interference signal levels for Experiment 2.

\subsection{Experiment 3: Three elements, one in- terference, desired signal, standard SMI, vary interference power}

Experiment 3 is a more complicated version of Experiment 1. Standard SMI and all three elements are used to suppress the first interference signal whose power level is varied. The desired signal is present with $\mathrm{SNR}(\operatorname{main})=16.5 \mathrm{~dB}$. Again, the desired signal level in the auxiliary elements is negligible. Figure 4.3 shows the results. For one pair of curves (theory and experiment), INR(aux1)-INR(main) is fixed at $6.5 \mathrm{~dB}$ whereas for the other pair it is fixed at $9.7 \mathrm{~dB}$. The difference in interference suppression between the two curve pairs is the result of increasing the auxiliary element gain. Thus, in the linear portion of the curves, a $3.2 \mathrm{~dB}$ $(=9.7-6.5)$ increase in auxiliary element gain has led to approximately a $5 \mathrm{~dB}$ increase in interference suppression for a given main element sidelobe level. Note again the lack of interference suppression for weak interference levels and low auxiliary element gain. For example, from the lower set of curves in 4.3 (corresponding to the lower gain auxiliary elements) we see that the array provides less than $10 \mathrm{~dB}$ interference suppression when $I N R($ main $)<-4 d B$. Theory and experiment show good agreement in 
Figure 4.3 especially in the middle range $-8 \mathrm{~dB}<\mathrm{INR}($ main $)<8 \mathrm{~dB}$. Above this range it becomes difficult to measure the small output interference power.

Figure 4.4 shows the output SNR curves for Experiment 3. Note that, as theory predicts, the SNR is maintained at the output.

\subsection{Experiment 4: Three elements, one in- terference, desired signal, standard SMI, vary main antenna sidelobe}

In the fourth experiment (Figure4.5) using standard SMI, the first interference signal is present, the first auxiliary element gain (INR(aux1)) is fixed, INR(aux2) = INR(main) $-12 \mathrm{~dB}$, and the main element sidelobe level is allowed to vary. The second interference is off and the desired signal is present with $\operatorname{SNR}($ main $)=16.06 \mathrm{~dB}$. The top pair of curves are theory and experiment for $\operatorname{INR}(\operatorname{aux} 1)=7.63 \mathrm{~dB}$, and the middle and lower pair are for $\operatorname{INR}(\operatorname{aux} 1)=4.20 \mathrm{~dB}$ and $1.20 \mathrm{~dB}$, respectively. The discrepancy between theory and experiment in the top pair of curves is explained, again, by the difficulty in measuring such small output interference powers. In the measureable regions, however, results are quite good. It is interesting to note that when the interference level in the main element is small relative to that in the auxiliary element (i.e. the main element sidelobe level is small compared to the auxiliary element gain), the theoretical interference suppression is relatively independent of the sidelobe level. From Figure 4.5 we see that for low-gain auxiliary antennas $(\operatorname{INR}(\operatorname{aux} 1)<1.20 \mathrm{~dB})$ and 


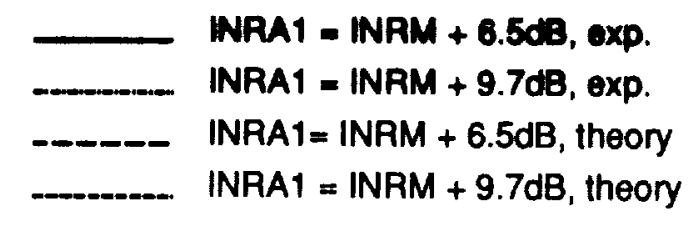

\section{ELEMENTS, I1 AND DESIRED, SNRM $=16.50 \mathrm{~dB}$}

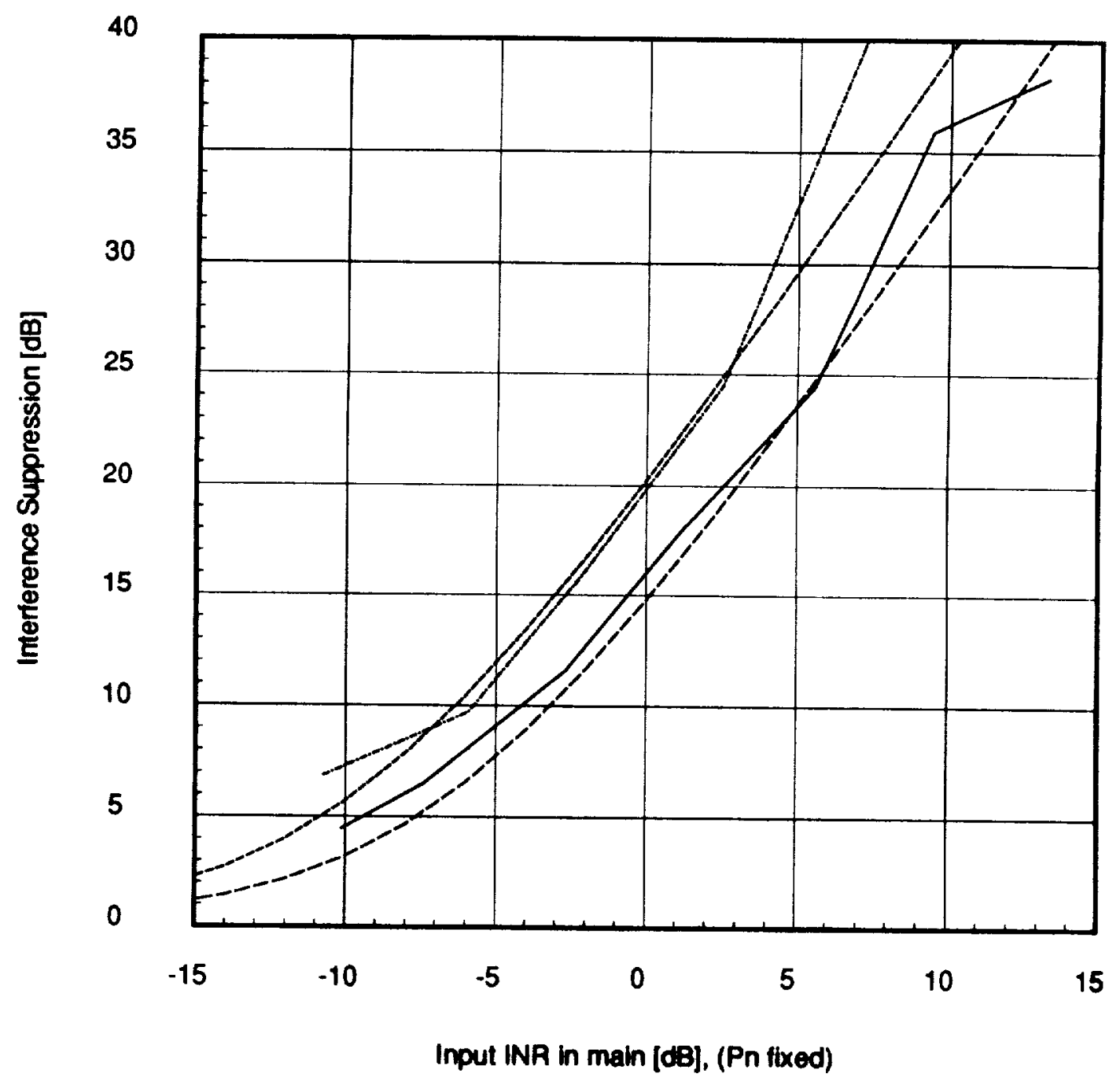

Figure 4.3: Experiment 3: Suppression of the first interference signal with a 3-element array, $\mathrm{SNR}$ (main) $=16.5 \mathrm{~dB}$. The top pair of curves are theory and experiment for fixed INR(aux 1$)-\operatorname{INR}($ main $)=9.7 \mathrm{~dB}$. The bottom pair are for $\operatorname{INR}(\operatorname{aux} 1)-\operatorname{INR}($ main $)=6.5 \mathrm{~dB}$. 


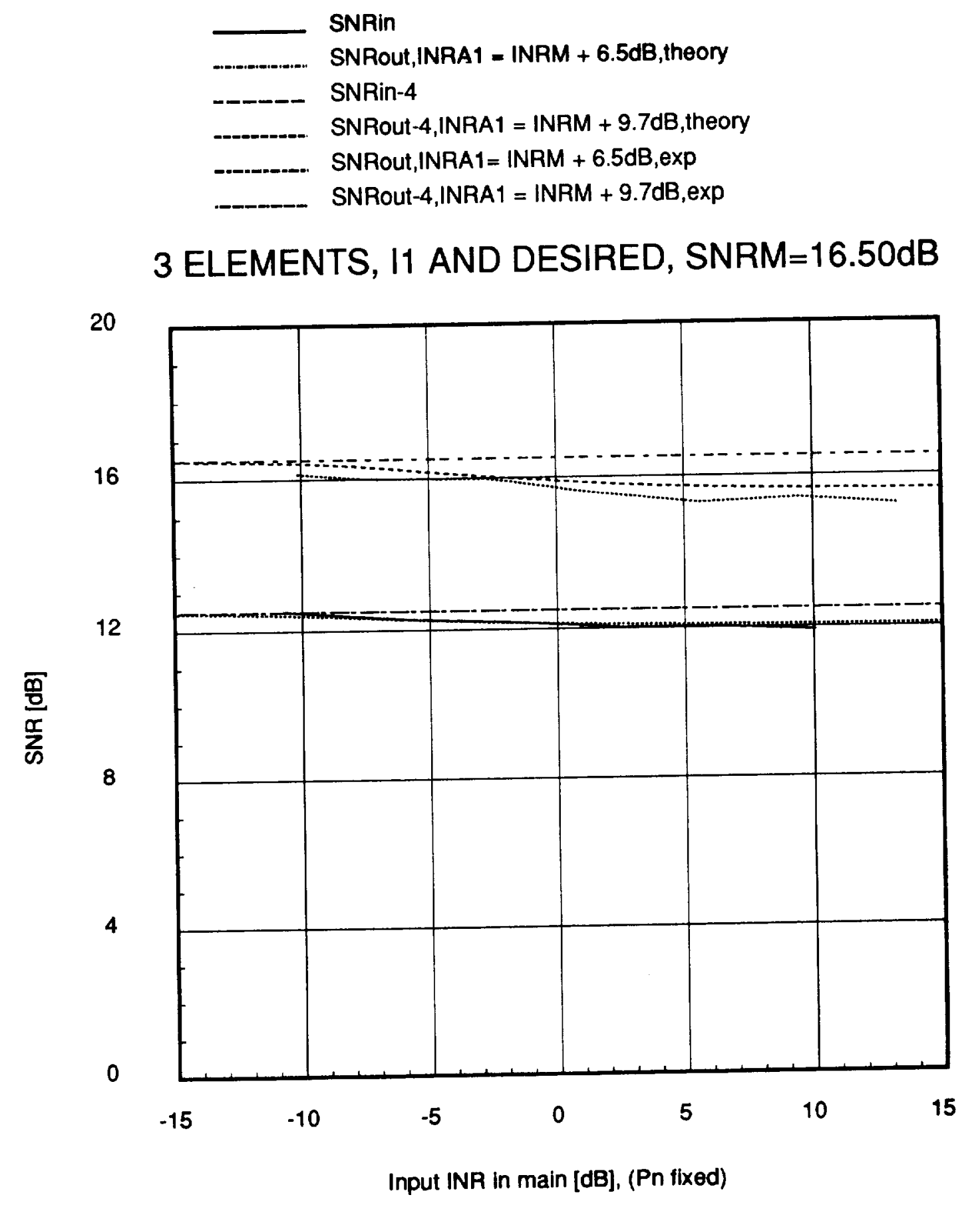

Figure 4.4: SNR plots for Experiment 3: The top three curves are input SNR(main), theoretical SNR(out), and experimental SNR(out) for $\operatorname{INR}(\operatorname{aux} 1)=\operatorname{INR}($ main $)+6.5 \mathrm{~dB}$. The bottom three curves are the same for INR(aux 1$)=\operatorname{INR}($ main $)+9.7 \mathrm{~dB}$ and have been displaced $-4 \mathrm{~dB}$ for clarity. 
weak interference in the main element (INR(main) $<-5 \mathrm{~dB})$, there is less than 7.5dB interference suppression. Figure 4.6 shows the input SNR(main) and the three SNR(out) curves for Experiment 4. There is little or no loss in SNR from input to output. This experiment and the last will help us to quantitatively assess the value of using modified SMI.

\subsection{Experiments 5 and 6: Three elements, one interference, desired signal, fixed in- put powers, modified SMI, vary fraction F}

In the above experiments, we observed a lack of weak interference suppression in the case of low-gain auxiliary antennas. The purpose of Experiments 5 and 6 is to observe interference suppression when modified SMI is applied to such cases. In both of these experiments all 3 elements are used and only the first interference and desired signal are present. The results are plots of interference suppression and SNR as the fraction F of (3.3) is varied. Table 4.2 presents the input signal levels for the two experiments. INR(main) is fixed at $-9.45 \mathrm{~dB}$ in Experiment 5 and is $-2.68 \mathrm{~dB}$ in Experiment 6. Figures 4.7 and 4.8 show the results of Experiment 5 and Figures 4.9 and 4.10 are the results of Experiment 6. The SNR plots show input SNR(main) and theoretical and experimental SNR(out) and are presented "waterfall style" so that the curves do not lay atop one another (i.e. the SNR curves corresponding to the top pair of interference curves have been displaced $-4 \mathrm{~dB})$. 
One interference, desired $S N R M=16.06 \mathrm{~dB}$, vary main sidelobe

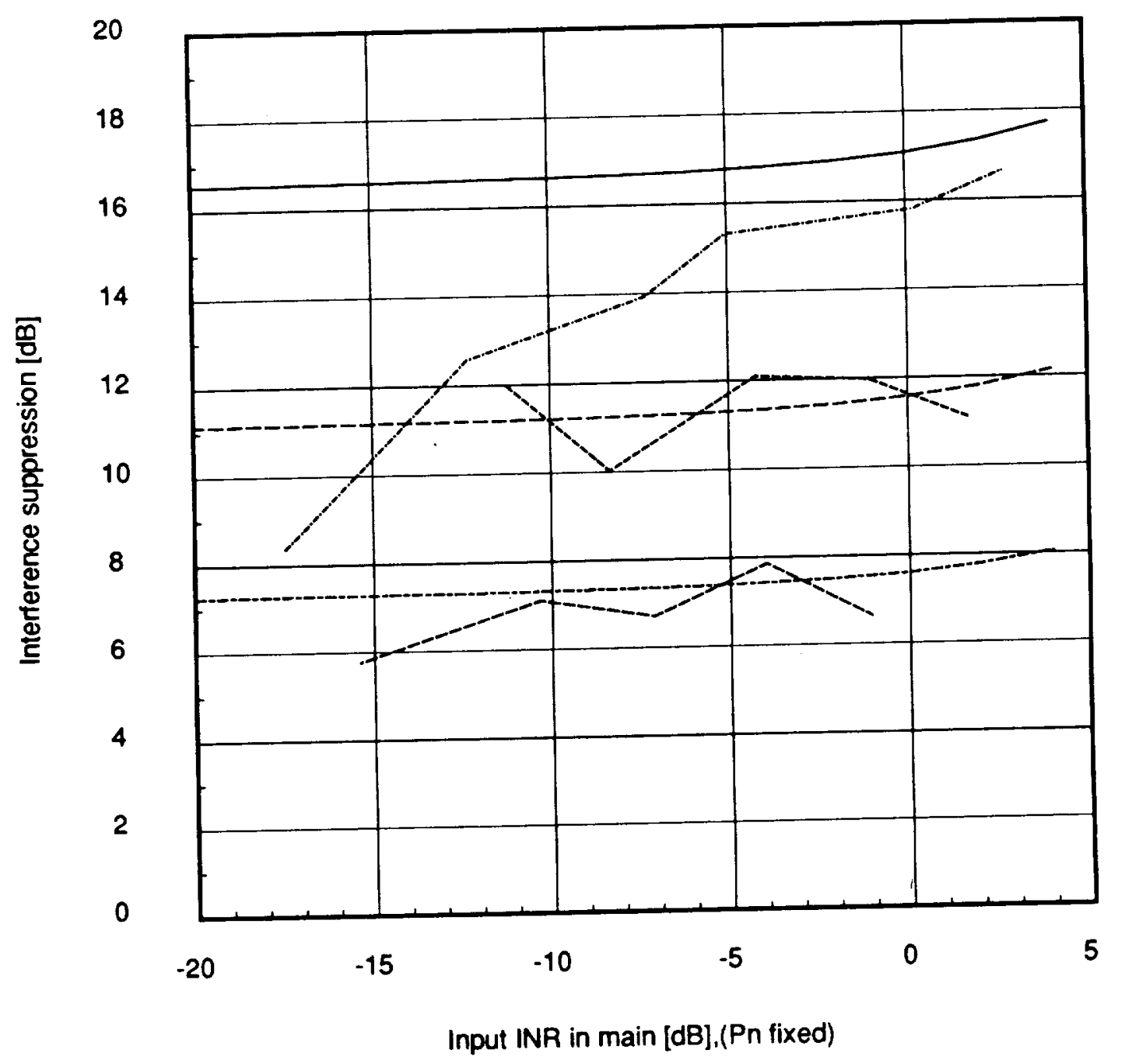

Figure 4.5: Experiment 4: Suppression of the first interference signal with a 3-element array, $\operatorname{SNR}($ main $)=16.06 \mathrm{~dB}$. The first auxiliary element gain is fixed and the main element sidelobe varies. The top pair of curves are theory and experiment for fixed INR(aux1)=7.63dB. The middle and bottom pairs are for $\operatorname{INR}(\operatorname{aux} 1)=4.20 \mathrm{~dB}$ and $1.20 \mathrm{~dB}$, respectively. 


\section{SNRin}

SNRout, i1 nra $1=7.63 \mathrm{~dB}$, exper.

SNRout, i1 nra1 $=4.20 \mathrm{~dB}$, exper.

SNRout, i1 $1 \mathrm{nra} 1=1.20 \mathrm{~dB}$, exper.

One interference, desired $\mathrm{SNRM}=16.06 \mathrm{~dB}$, vary main sidelobe

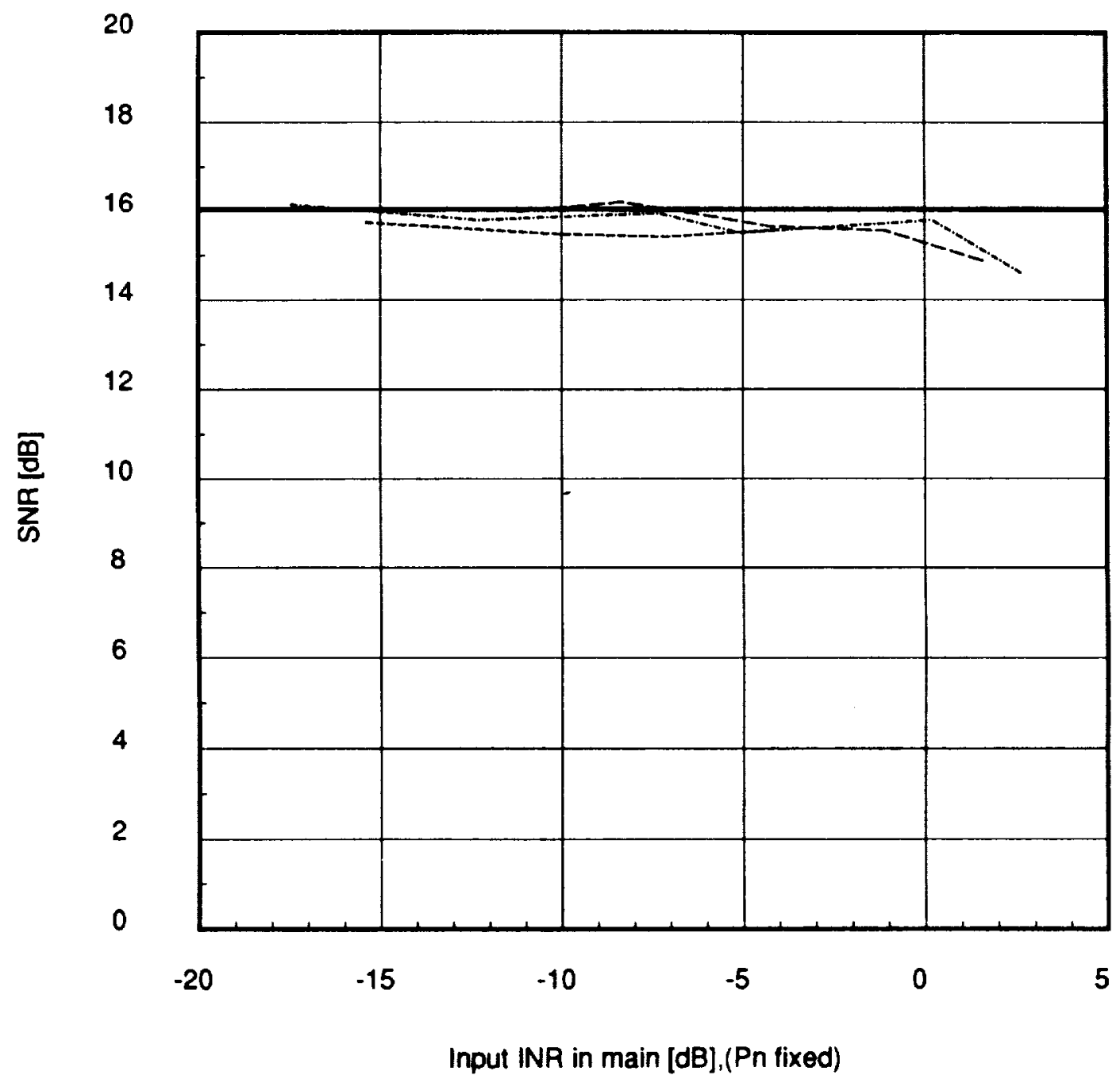

Figure 4.6: SNR(main) and SNR(out) curves for Experiment 4. 


\begin{tabular}{||l||r|r|r|r||}
\hline \hline & INR(main) & SNR(main) & \multicolumn{2}{|c|}{ INR(aux1) } \\
\cline { 4 - 5 } & & & $\begin{array}{r}\text { top pair } \\
\text { of curves }\end{array}$ & $\begin{array}{c}\text { bottom pair } \\
\text { of curves }\end{array}$ \\
\hline \hline Exp. 5 & -9.45 & 16.50 & 0.43 & -3.57 \\
\hline Exp.6 & -2.68 & 16.50 & 4.72 & 0.53 \\
\hline \hline
\end{tabular}

Table 4.2: Signal levels for Experiments 5 and 6.

In all cases theory and experiment compare favorably. From Figures 4.7 and 4.9 we see that interference suppression increases as the fraction $F$ used in the modified SMI algorithm is increased from zero towards one. For example, in Figure 4.7 with $\operatorname{INR}(\mathrm{aux} 1)=0.43 \mathrm{~dB}$ we see that interference suppression is increased $9.5 \mathrm{~dB}$ by changing from $\mathrm{F}=0.0$ (standard $\mathrm{SMI}$ ) to $\mathrm{F}=0.8$. One can also make the observation from Figure 4.7 that a modified SMI array with low-gain auxiliary antennas $(\operatorname{INR}(\operatorname{aux} 1)=-3.57)$ and $\mathrm{F}=0.6$ performs the same with respect to interference suppression as a standard SMI array with auxiliary antennas of $4 \mathrm{~dB}(=0.43+3.57)$ higher gain. Similar observations can be made about Figure 4.9 of Experiment 6 . From the SNR plots of Figures 4.8 and 4.10 we see that for $\mathrm{F}<0.9$ there is less than a $2.5 \mathrm{~dB}$ loss of SNR from input to output. Experiments 5 and 6 have demonstrated that modified SMI yields increased suppression of weak interference with only a small loss in desired signal power. 


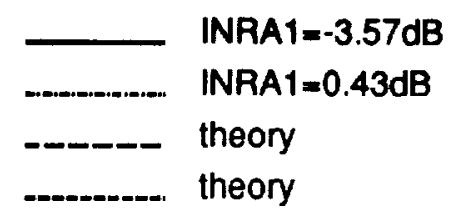

Mod. SMI, INRM=-9.45dB, SNRM=16.50dB, f-gain tradeoff

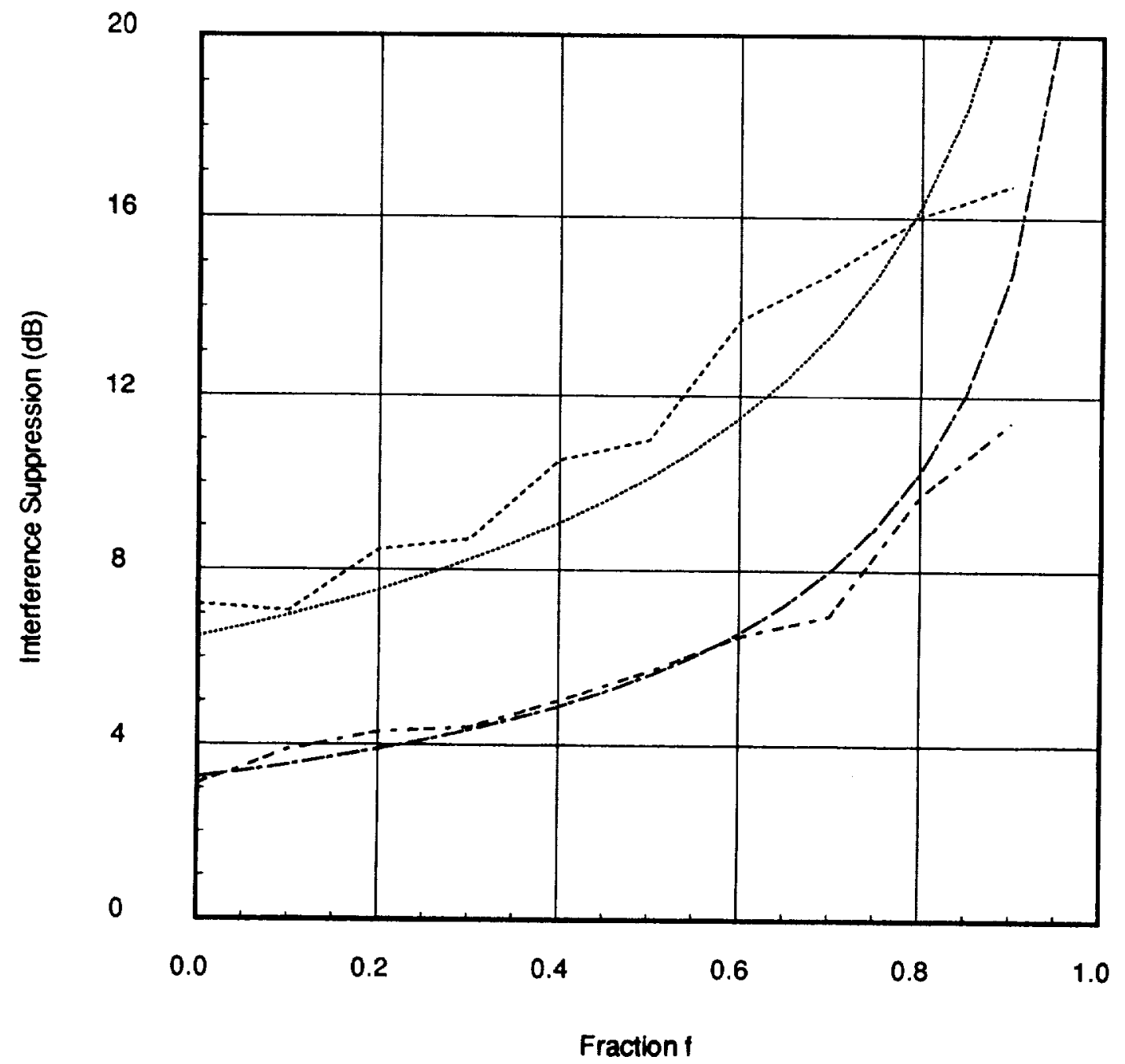

Figure 4.7: Experiment 5: Suppression of the first interference signal versus fraction F using a 3-element modified SMI array with desired SNR(main) = $16.50 \mathrm{~dB}$. INR(main) $=-9.45 \mathrm{~dB}$. Bottom pair of curves has $\operatorname{INR}(\operatorname{aux} 1)=$ $-3.57 \mathrm{~dB}$. Top pair has $\operatorname{INR}(\operatorname{aux} 1)=0.43 \mathrm{~dB}$. $\operatorname{INR}(\operatorname{aux} 2)=-20 \mathrm{~dB}$. 


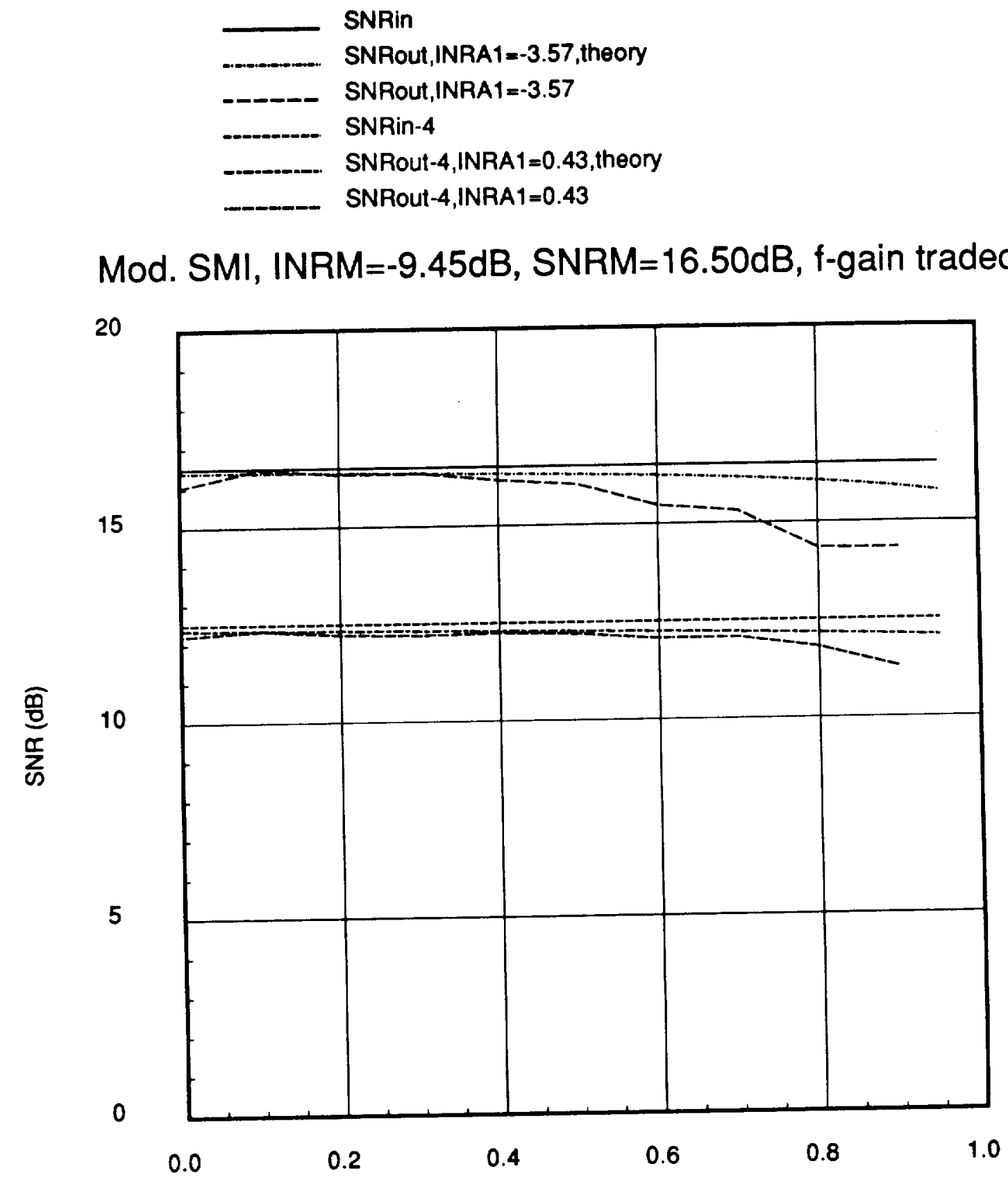

Fraction $f$

Figure 4.8: SNR curves for Experiment 5. Top three curves are input SNR(main), theoretical SNR(out), experimental SNR(out) for INR(aux1)= $-3.57 \mathrm{~dB}$. Similarly, the bottom three curves are for $\operatorname{INR}(\operatorname{aux} 1)=0.43 \mathrm{~dB}$ and have been displaced $-4 \mathrm{~dB}$ for clarity. 

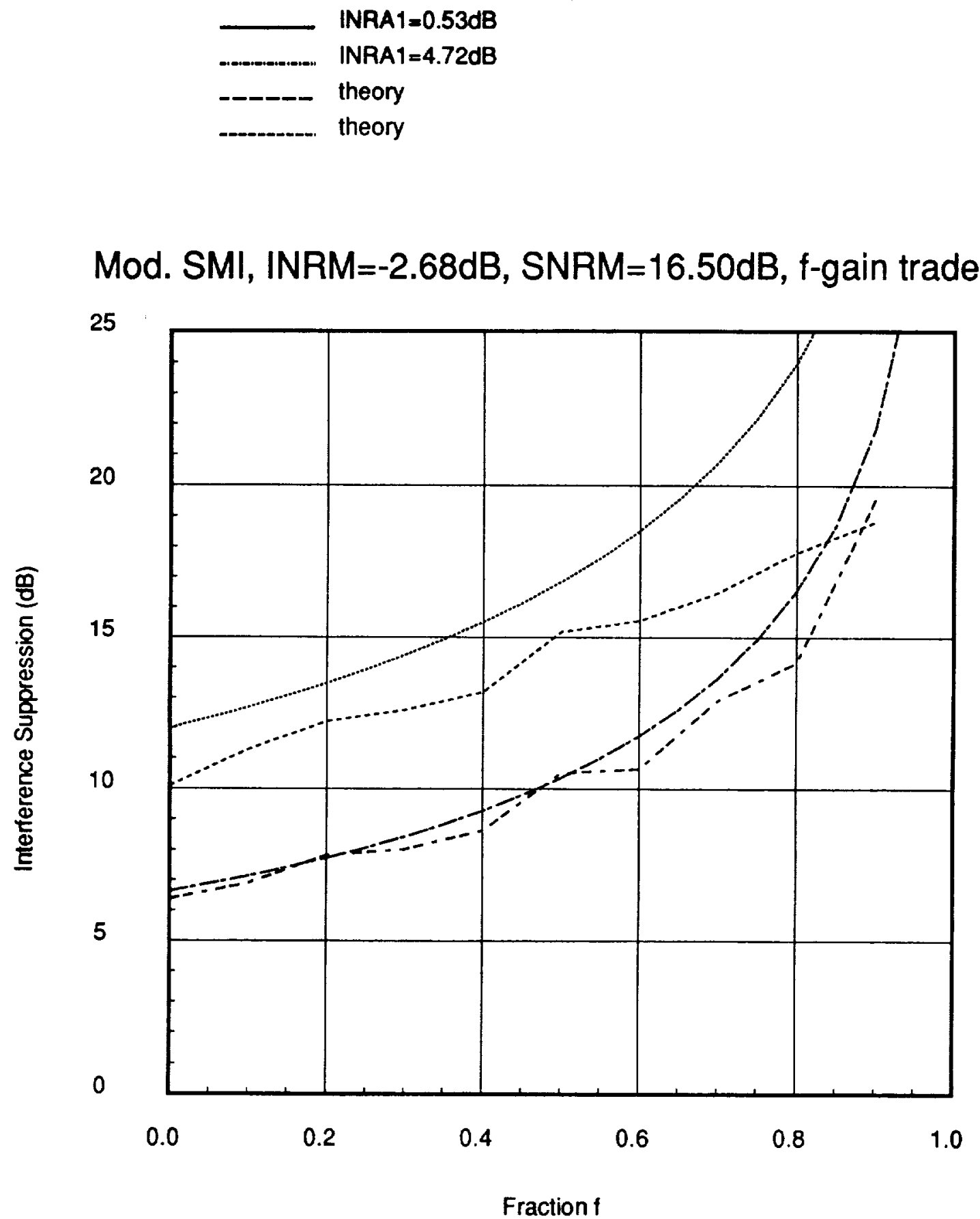

Figure 4.9: Experiment 6: Suppression of the first interference signal versus fraction $F$ using a 3-element modified SMI array with desired SNR(main) = $16.50 \mathrm{~dB}$. INR(main) $=-2.68 \mathrm{~dB}$. Bottom pair of curves has $\operatorname{INR}(\operatorname{aux} 1)=$ $0.53 \mathrm{~dB}$. Top pair has $\operatorname{INR}(\operatorname{aux} 1)=4.72 \mathrm{~dB}$. INR $(\operatorname{aux} 2)=-17 \mathrm{~dB}$. 


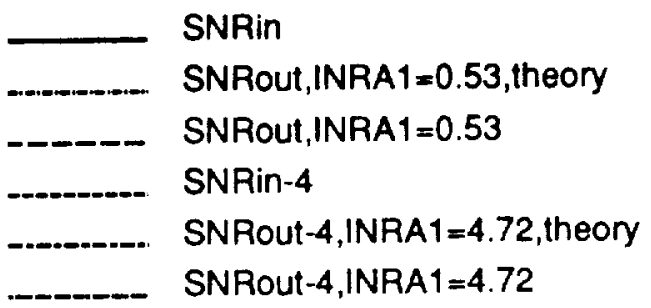

Mod. SMI, INRM $=-2.68 \mathrm{~dB}, \mathrm{SNRM}=16.50 \mathrm{~dB}, \mathrm{f}$-gain tradeoff

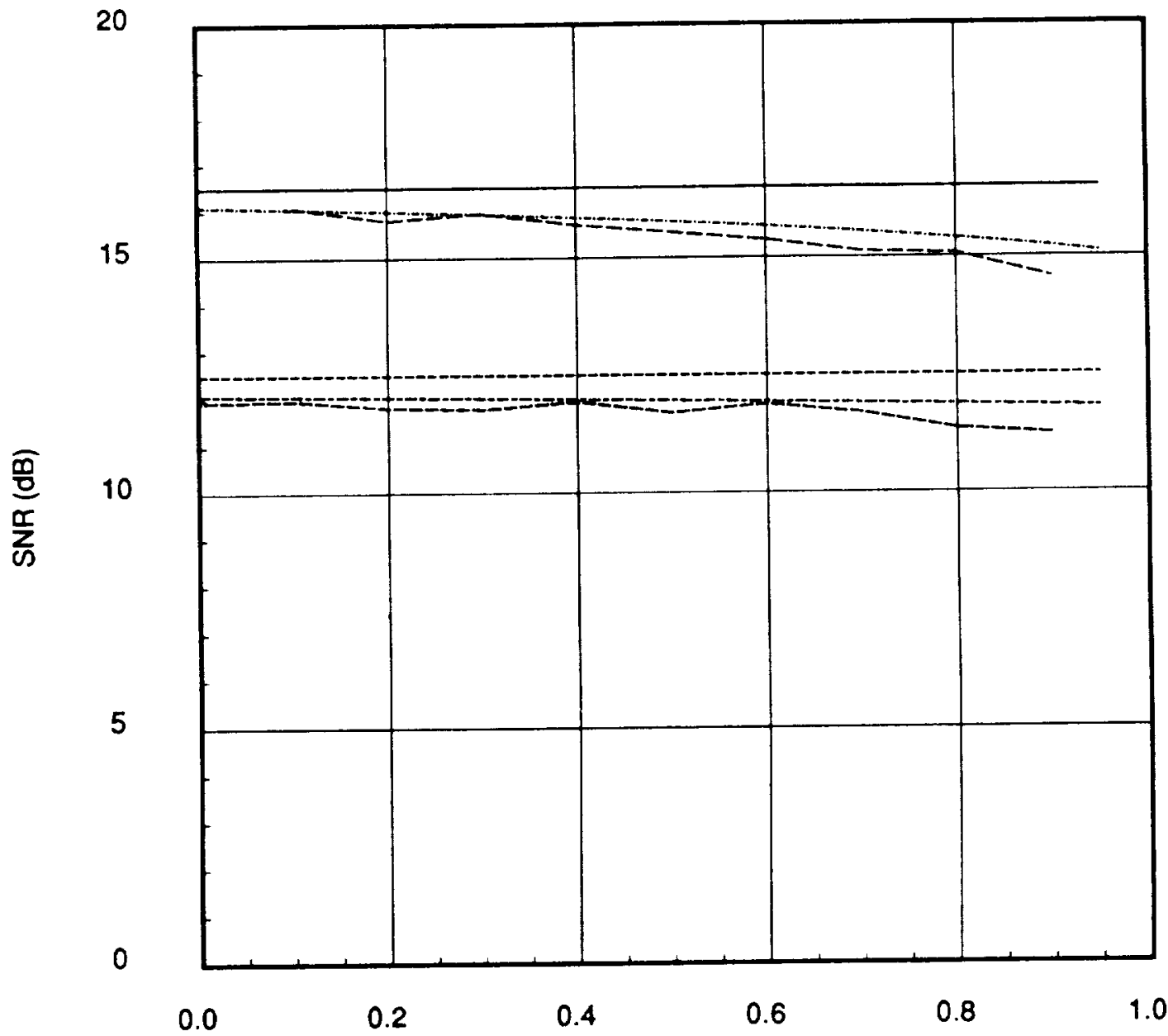

Fraction $f$

Figure 4.10: SNR curves for Experiment 6. Top three curves are input SNR(main), theoretical SNR(out), experimental SNR(out) for INR(aux1)= $0.53 \mathrm{~dB}$. Similarly, the bottom three curves are for $\operatorname{INR}(\operatorname{aux} 1)=4.72 \mathrm{~dB}$ and have been displaced $-4 \mathrm{~dB}$ for clarity. 


\section{Chapter 5}

\section{Conclusions}

The problem of weak interference signals entering a satellite - ground station communication link through the sidelobes of the receive antenna has been studied experimentally. The receive antenna is made adaptive so that antenna pattern nulls can be formed in the directions of the interference signals. Standard adaptive antennas which are designed to maximize the output SINR fail to achieve adequate suppression of weak interference, especially when the auxiliary antenna elements have low gain. In a previous solution to this problem, the feedback loops used to control the array weights were modified so that the two inputs to the feedback loop correlators had uncorrelated noise components. These modified feedback loops yielded weights which were less influenced by noise and as a result adapted to null the interference. This solution had the drawback of requiring either two antennas per auxiliary element, two amplifiers per auxiliary element, or both.

In this report, an alternative solution using a modified SMI algorithm is studied experimentally. The modification involves subtracting a fraction of 
the minimum eigenvalue of the estimated covariance matrix from its diagonal elements before inversion. The modified covariance matrix resembles a standard covariance matrix formed in a less noisy environment and thus "frees" the weights to further suppress the interference. The SMI modification is made in software and thus has the advantage of not requiring any additional antennas or amplifiers.

The modified SMI algorithm is implemented on a three-element experimental array system. The need for a weight (vector modulator) in the main channel is eliminated by normalizing the estimated weight vector so that the main channel weight is unity. Sampling of the main channel is accomplished by sampling the array output while the auxiliary element weights are set to zero. The signal snapshots are the input to the SMI algorithm and are formed from scaled samples. Such scaling provides a coarse adjustment for the losses present in the experimental system. The modified SMI algorithm is presented in detail. In order to quantify the performance of the experimental system the signal powers in each channel are estimated from samples. This is made possible by a special pulse modulation scheme in which the desired and interference signals are separated in time. Finally, a fully-automated, iterative procedure for system calibration is presented. In this procedure, amplitude and phase scale factors for the auxiliary signal samples are determined by comparing the weights that null the interference in a simple signal scenario with those that are estimated by the algorithm.

The experiments that are presented in this report focus on steady state performance (i.e. a large number of snapshots are used to form the weight estimate for any particular scenario). In all cases, the experimental results 
compare favorably with theoretical predictions based on the ideal array model. As expected, the interference suppression increases as the power of the interference signal incident on the array increases. In particular, it is noted that interference suppression is relatively independent of the main antenna sidelobe level when that sidelobe level is small relative to the auxiliary element gain. In other words, the interference suppression is to a great extent determined by the gain of the auxiliary antennas in such situations. When low-gain auxiliary elements are used, standard SMI leads to only small amounts of interference suppression. When modified SMI is used the suppression is increased by as much as $13 \mathrm{~dB}$ in the experiments. Thus, for large numbers of snapshots the experimental modified SMI array can provide the desired performance as predicted by theory. 


\section{Bibliography}

[1] R.T. Compton, Jr., Adaptive Antennas - Concepts and Performancd. Englewood Clifs, NR: Prentice-Hall, 1988.

[2] I.J. Gupta and A.A. Ksienski, "Adaptive arrays for weak interfering signals," IEEE Transactions on Antennas and Propagation, vol. AP. 34, no. 3, pp. 420-426, March 1986.

[3] J. Ward, E.K. Walton, I.J. Gupta, and A.A. Ksienski, "An experimental adaptive array to suppress weak interfering signals," IEEE Transactions on Antennas and Propagation," vol. 36, no. 11, pp. 1551-1559, November 1988.

[4] J. Ward, "Adaptive arrays for weak interfering signals -an experimental system," M.S. Thesis, The Ohio State University, Summer 1987.

[5] I.J. Gupta, "SMI adaptive antenna arrays for weak interfering signals," IEEE Transaction on Antennas and Propagation, vol. AP-34, no. 10, pp. 1237-1242, October 1986.

[6] R. Dilsavor and R. Moses, "Analysis of modified SMI method for adaptive array weight control," Technical Report 716111-6, The Ohio State University, Department of Electrical Engineering, ElectroScience Laboratory, February 1989.

[7] K. Steadman, "Adaptive array for weak interfering signals - Geostationary satellite experiments," M.S. Thesis, The Ohio State University, Summer 1989, prepared under Grant NAG 3-536, NASA Lewis Resarch Center.

[8] H.L. Van Trees, Detection, Estimation, and Modulation Theory: Part I. New York: Wiley, 1968. 
[9] R.E. Ziemer and W.H. Tranter, Principles of Communications Systems, Modulation, and Noise. Boston: Houghton Mifflin Company, 1985. 


\section{Appendix A}

\section{Experimental System Software (Fortran IV)}

c

MASTER PROGRAY FOR RUWIIIG ADAPTIVE ARALY SYSTEH COHAO/OUTDTA/X,AVE, AVCOR, OFF,

$X$ LMA , HMAO,LPH, BPH, ISC, CPHI

COHHO/CTST/AVCD, AVIF, AVISE, ST 1

CORHON/SCHRO/LATT, LPHI, PI

COMOH/WTBLX/LDAO, WI , WQ, VHX, VMY , WN

COYMOI/SPLRS/PUR, PUSE, ITER, IFL, OFLAG, IFL 10, IIS , IIST

COMHOV/SHI/COV,, $\operatorname{COV}$, ICOV $1, \mathrm{MCOV} 10, \mathrm{ICT}$

COHPLEX X(3,64), 1 COR (2), OFF (3), $\operatorname{VVCD}(2), \operatorname{AVIF}(2), \operatorname{AVISE}(2)$

COAPLEX $\operatorname{cov}(3,3), \operatorname{cov} \operatorname{cop}(3,3), \operatorname{cov} I n V(3,3), \operatorname{sTBER}(3), 3 T 1$

COMPLBI CH( 3$), \operatorname{BVEC}(3,3), \mathrm{DET}, \operatorname{ID}(3,3), \mathrm{gTR}, \operatorname{CUTEST}, \operatorname{NEIOHT}(20,2)$

RELL VMX $(40,4)$, VHY $(40,4)$, POWERS $(20,4), \operatorname{ICOV}$

RELL EVLL (3), LVECT (3), HVECT (3), PIMIU, EIGVAL $(20,3)$

REAL PUR $(3,3), \operatorname{PISE}(3)$, PB (3), PA (3), AIS (3), AIST, CPMI (2)

REAL AVE $(6,3), \operatorname{LHG}(2), \operatorname{HMA}(2), \operatorname{LPH}(2), \operatorname{HPH}(2), P I, P(2), \operatorname{SIZE}$

REAL LPHI $(2,2), P M(4), W I(2), U Q(2), \operatorname{TEHPI}(2), \operatorname{TEMPQ}(2), U I O(2), U Q O(2)$

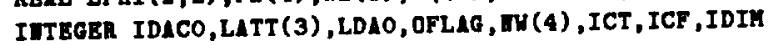

IITBGER ICOV 1, ICOV10

LOGICAL 1 FLIHE (6), FTYPE(4), $\operatorname{PLSPEC}(15), \operatorname{JKE}(8,4)$

DATA FTYPE/',', 'D', 'A', 'T'/CPBI/2*0.0/

DATA IDACO/"170440/0FF/3* $(0.0,0.0) /$

DATA WI/2*0.0/WQ/2*0.0/

DATA PI/3.1416927/MFLAG/O/

DATA ISC/1T/STEER/ $(0.0,0.0),(0.0,0.0),(1.0,0.0) /$

C Rouding data for VHOD control

CALL SCOPY ('VM1I.DAT', MME $(1,1)$ )

CALL SCOPY ('VM1Q.DAT', MHE $(1,2)$ )

CALL SCOPY ('VH2I.DAT', $\operatorname{IME}(1,3)$ )

CALL SCOPY ('VM2Q.DAT', WhE $(1,4)$ )

DO $15 \quad I=1,4$

OPEW (UTIT=10, WAHE= $I K E(1, I), T Y P E=$ ' OLD', FORH= 'UDFORHATTED')

DO $10 \mathrm{~J}=1,40$

$10 \operatorname{READ}(10, \operatorname{END}=11) \operatorname{VMx}(J, I), \operatorname{VMY}(J, I)$

11 W $(\mathrm{I})=\mathrm{J}$ 


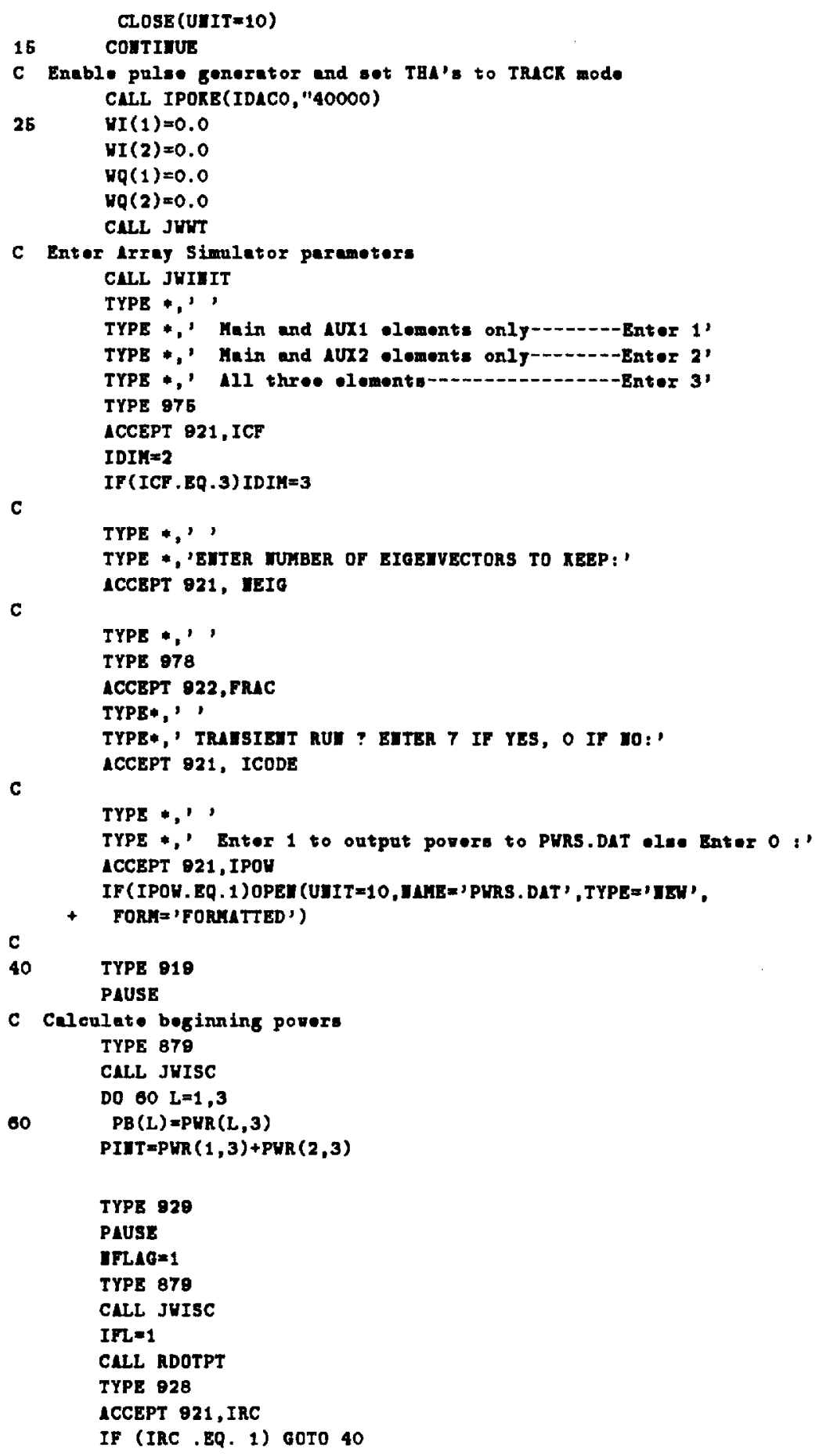




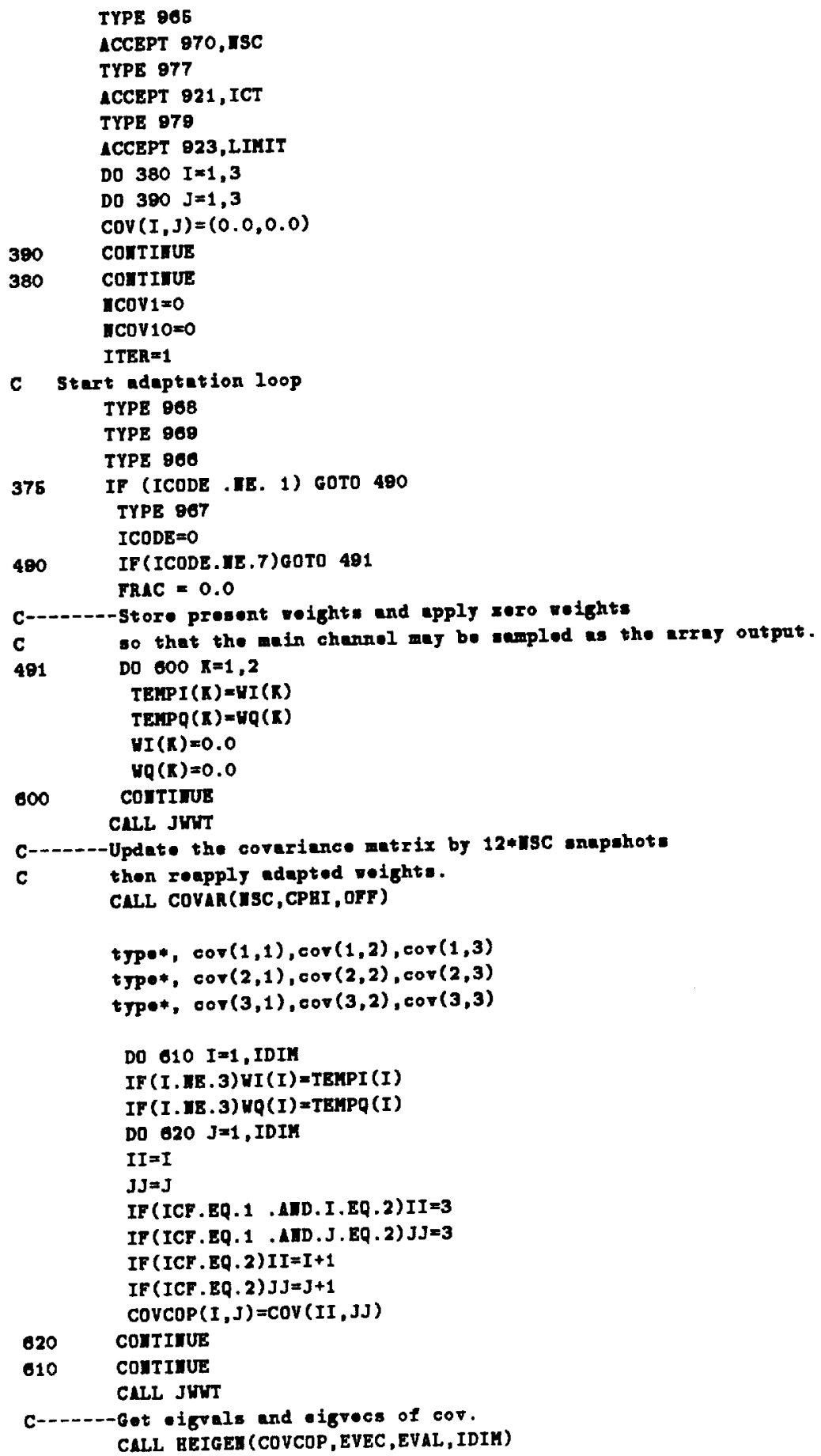




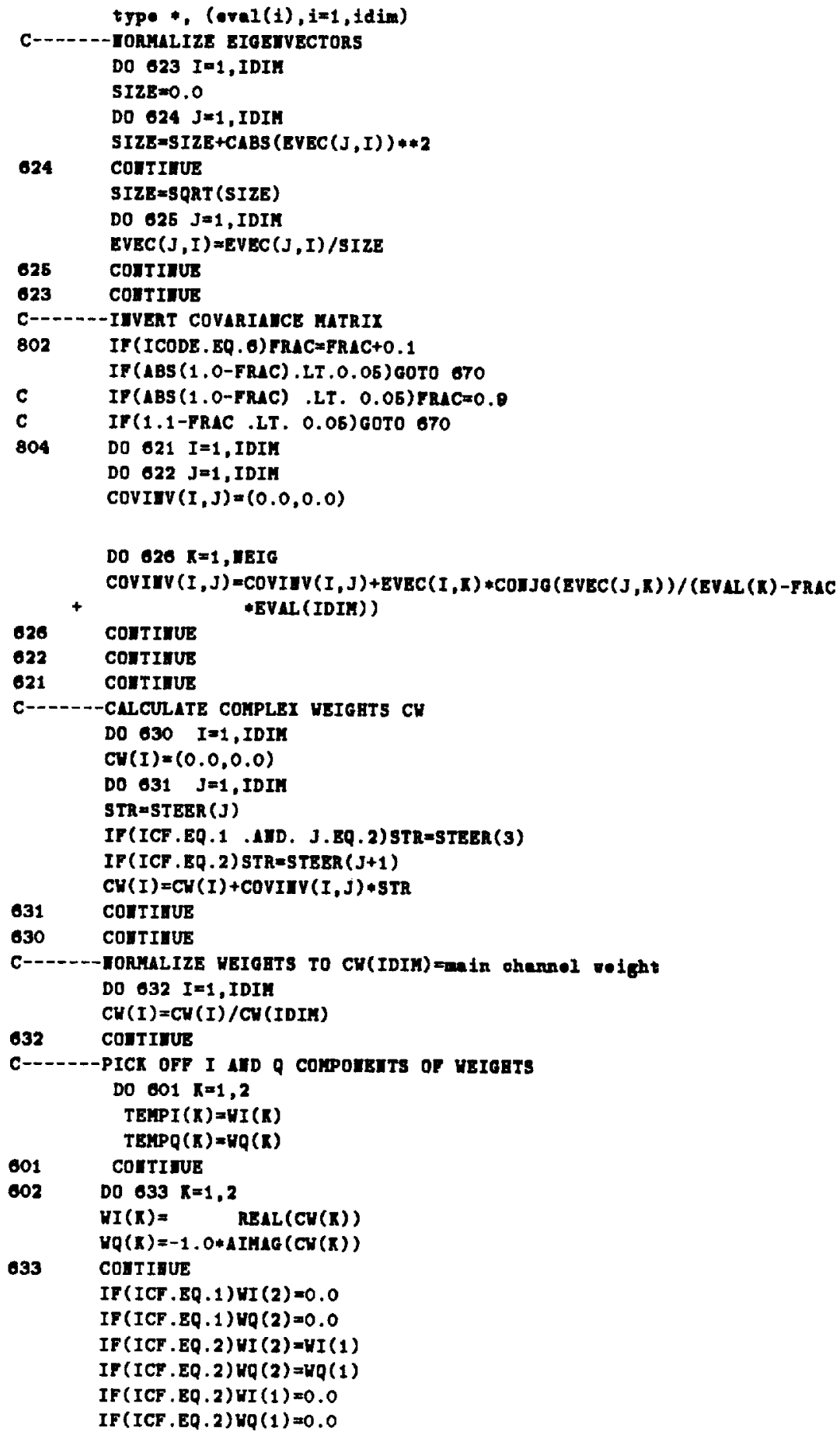




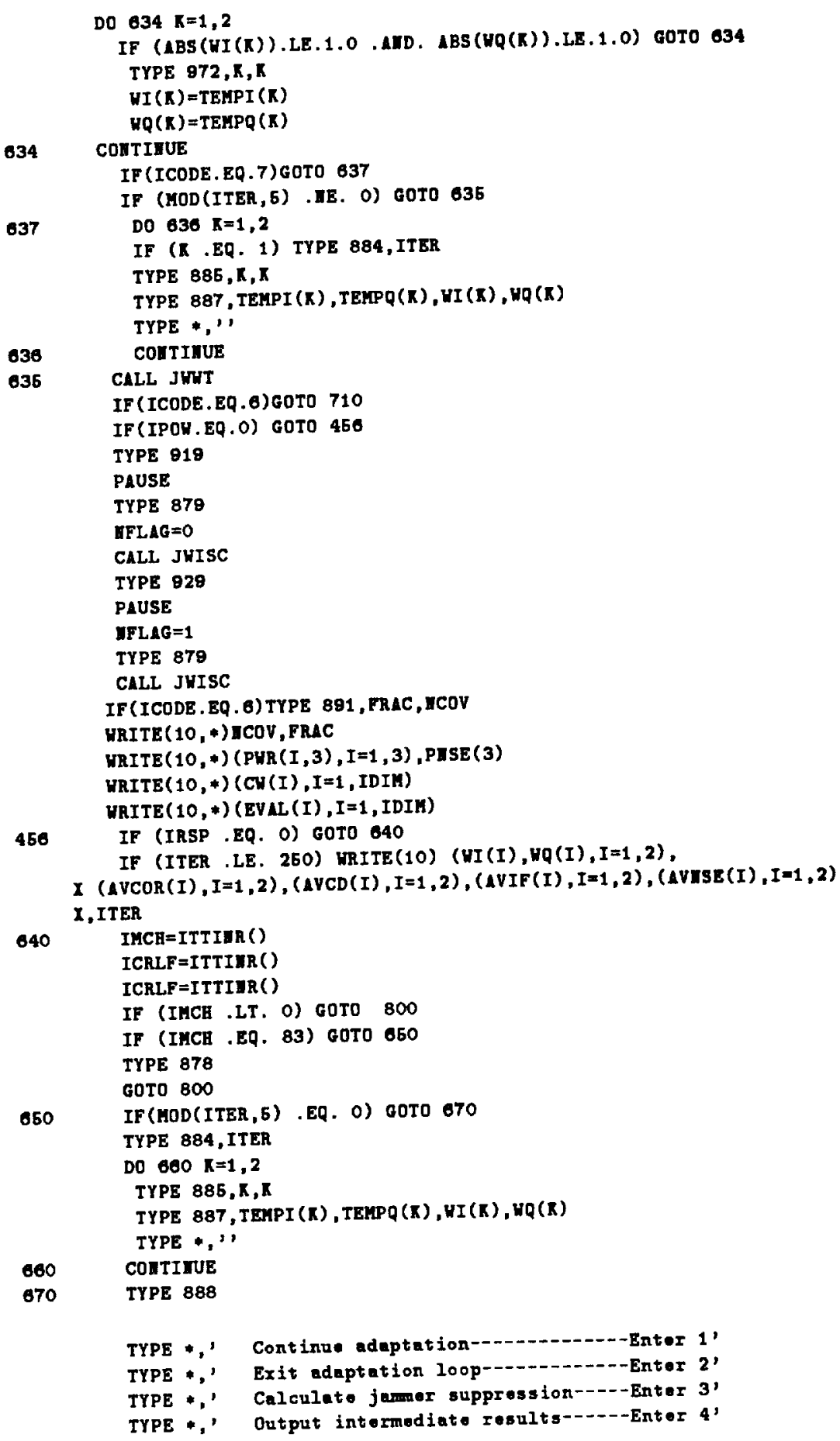




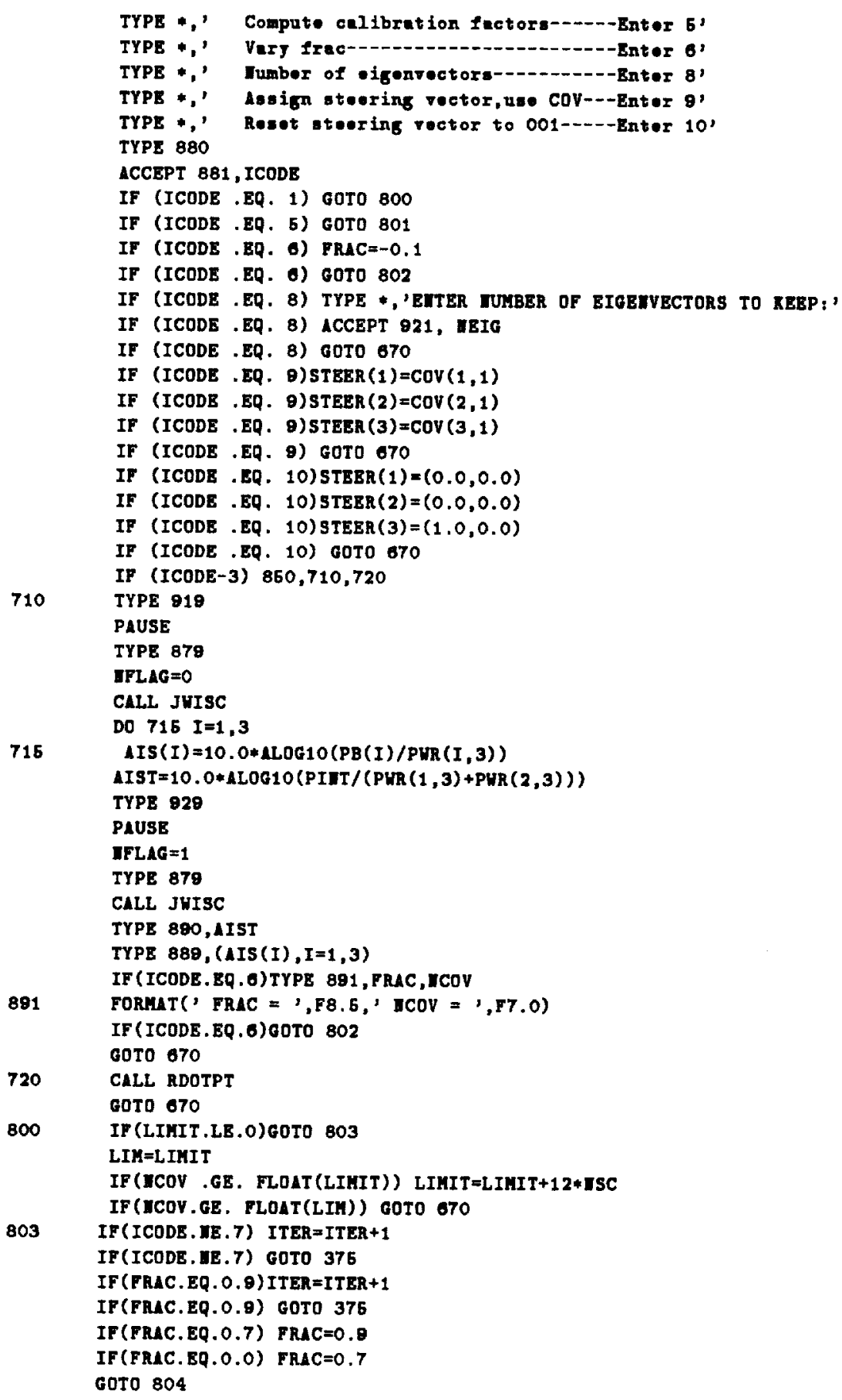




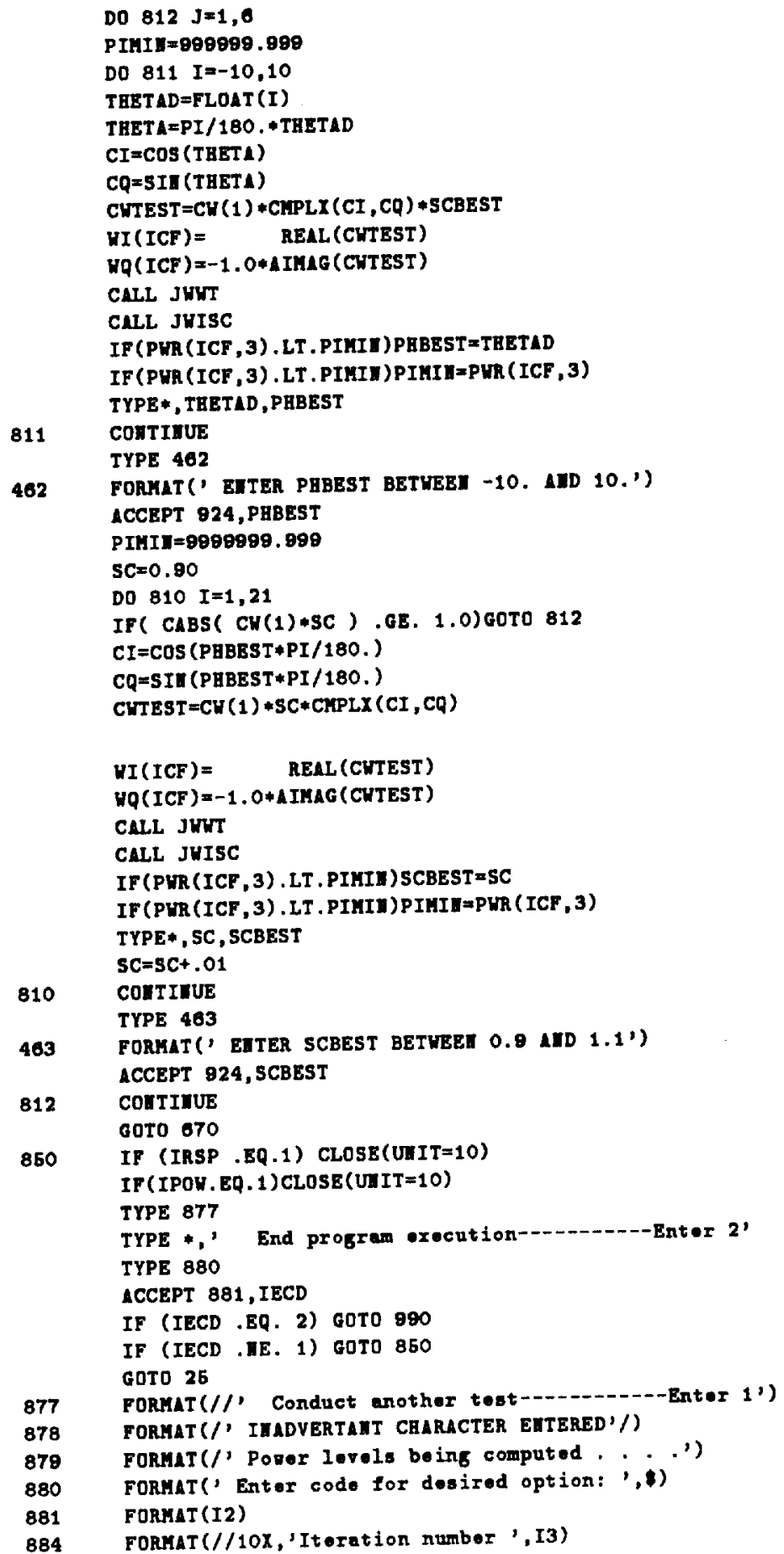




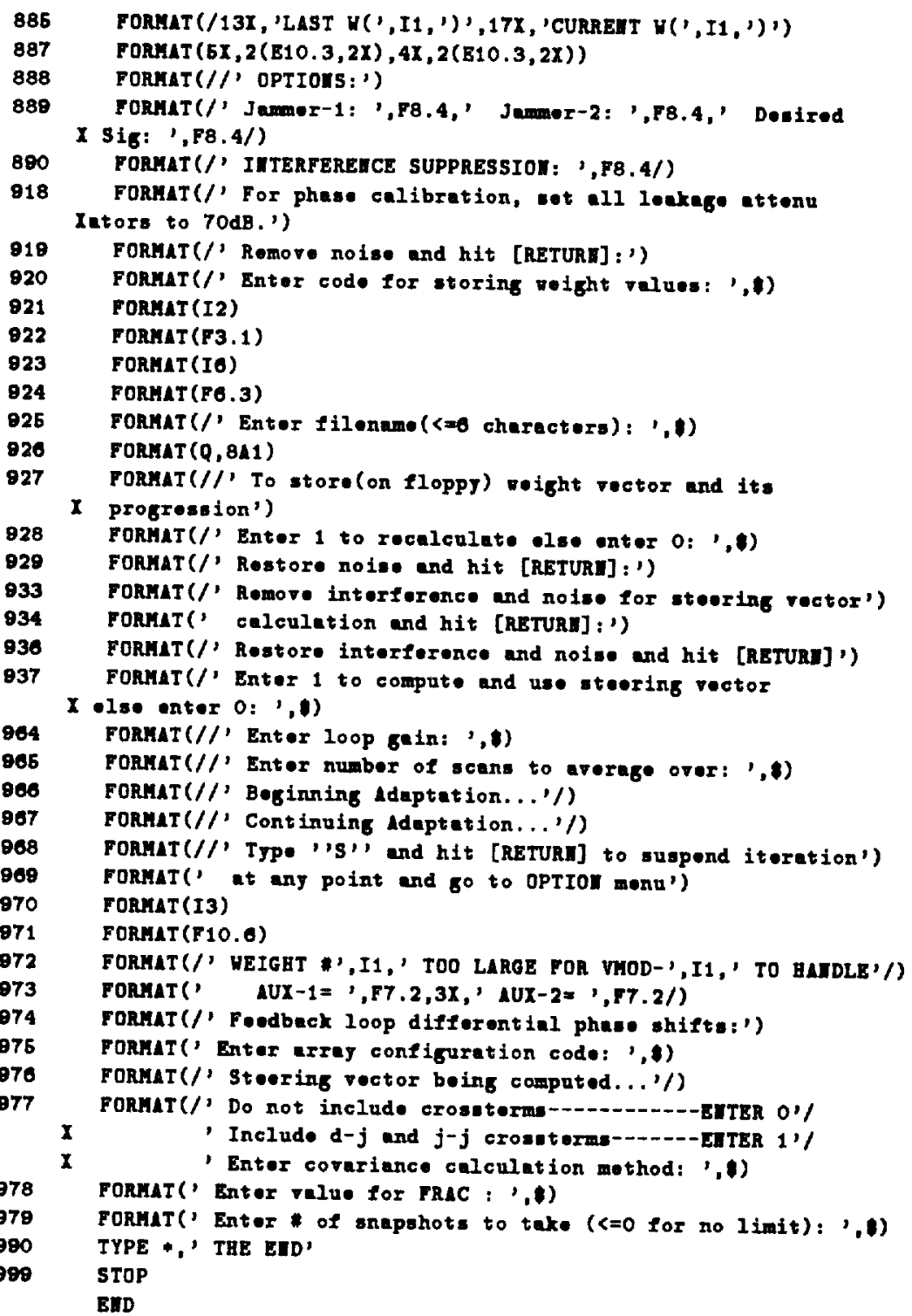

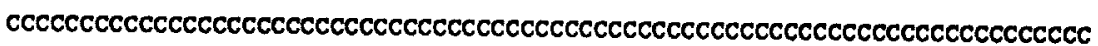

C Subprogram JUIIIT--sot erraj simulator parameter:

C Program to test control of programable ettenuntors of errey

C simulator using parallel output port (DRV11)

SUBROUTIIB JUIEIT

COMHON/SCHRO/LATT, LPHI, PW

IUTEGER DRBUF, IVAL, LATT (3), IH(3), IP (3)

AEAL LPHI $(2,2), P H(4)$ 


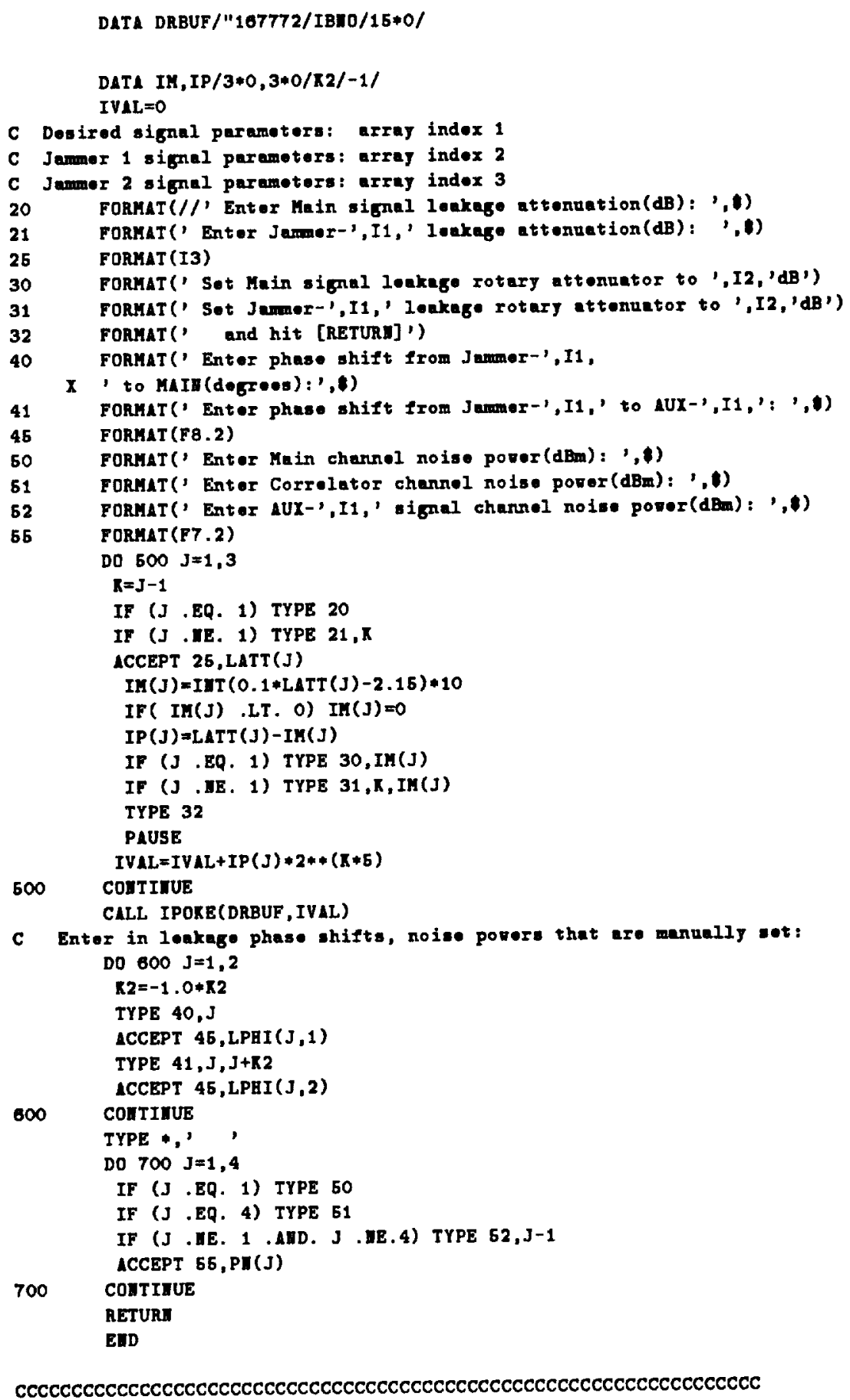




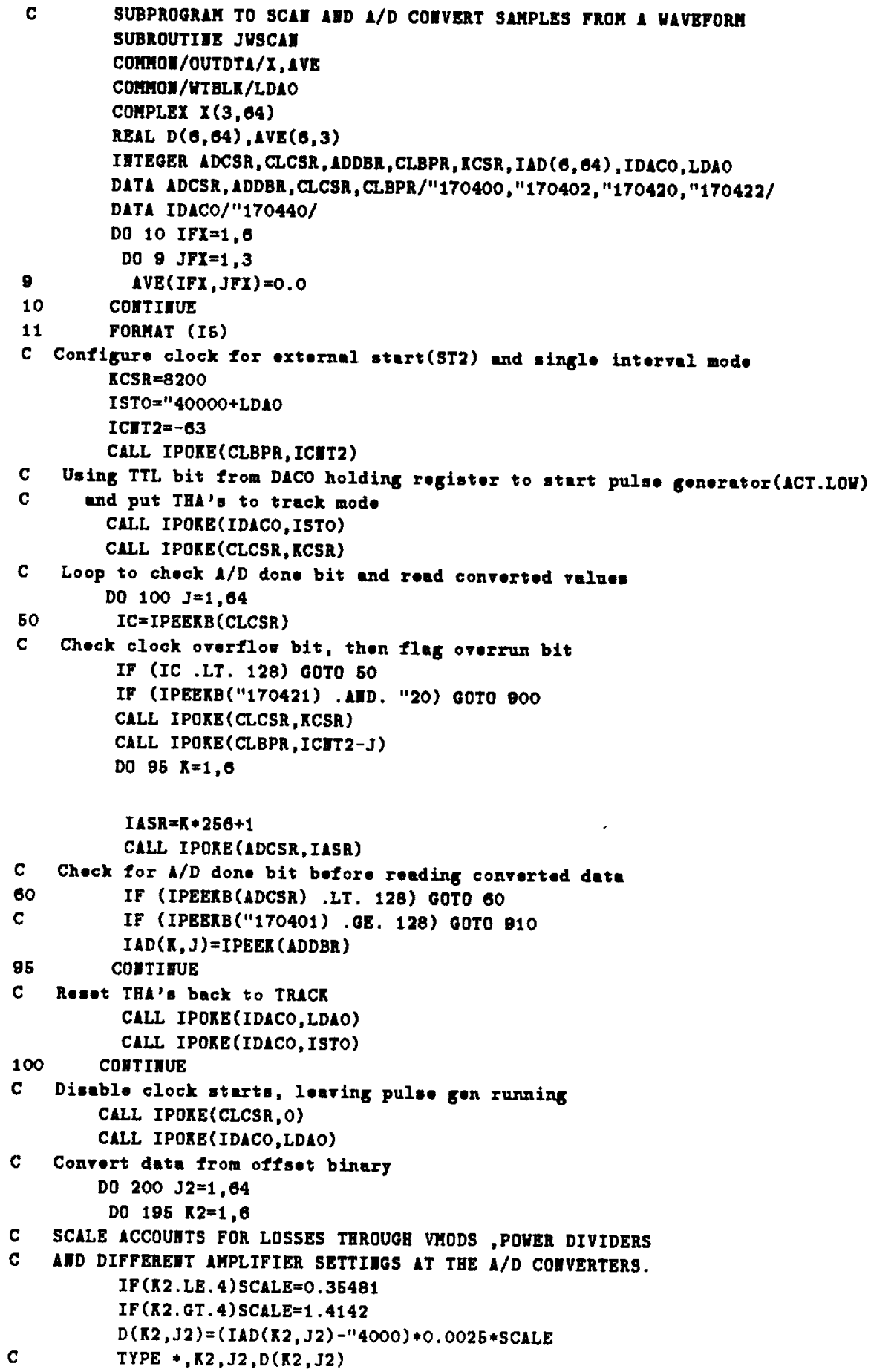




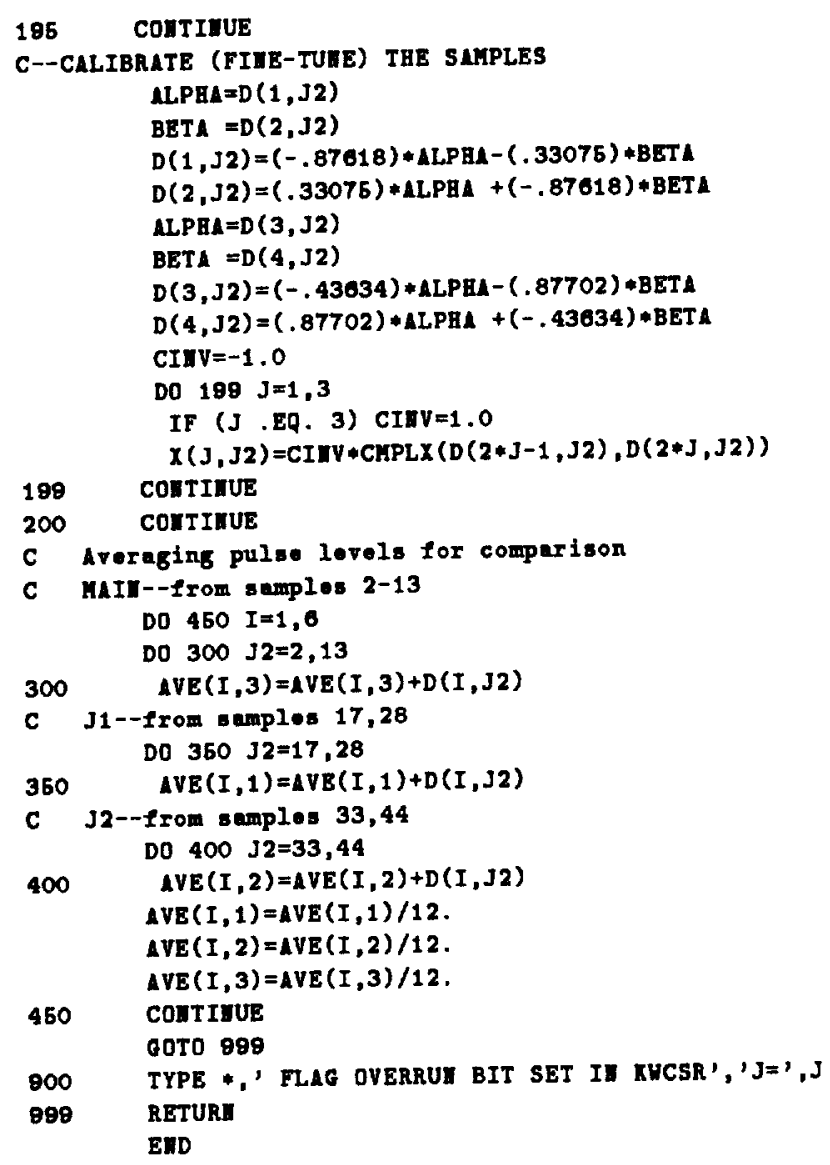

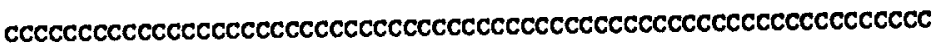

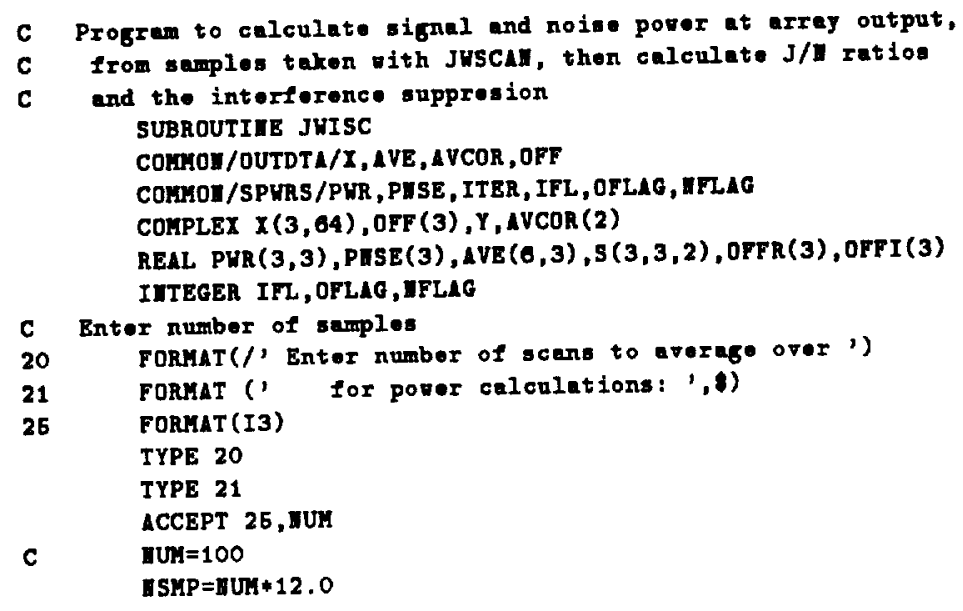




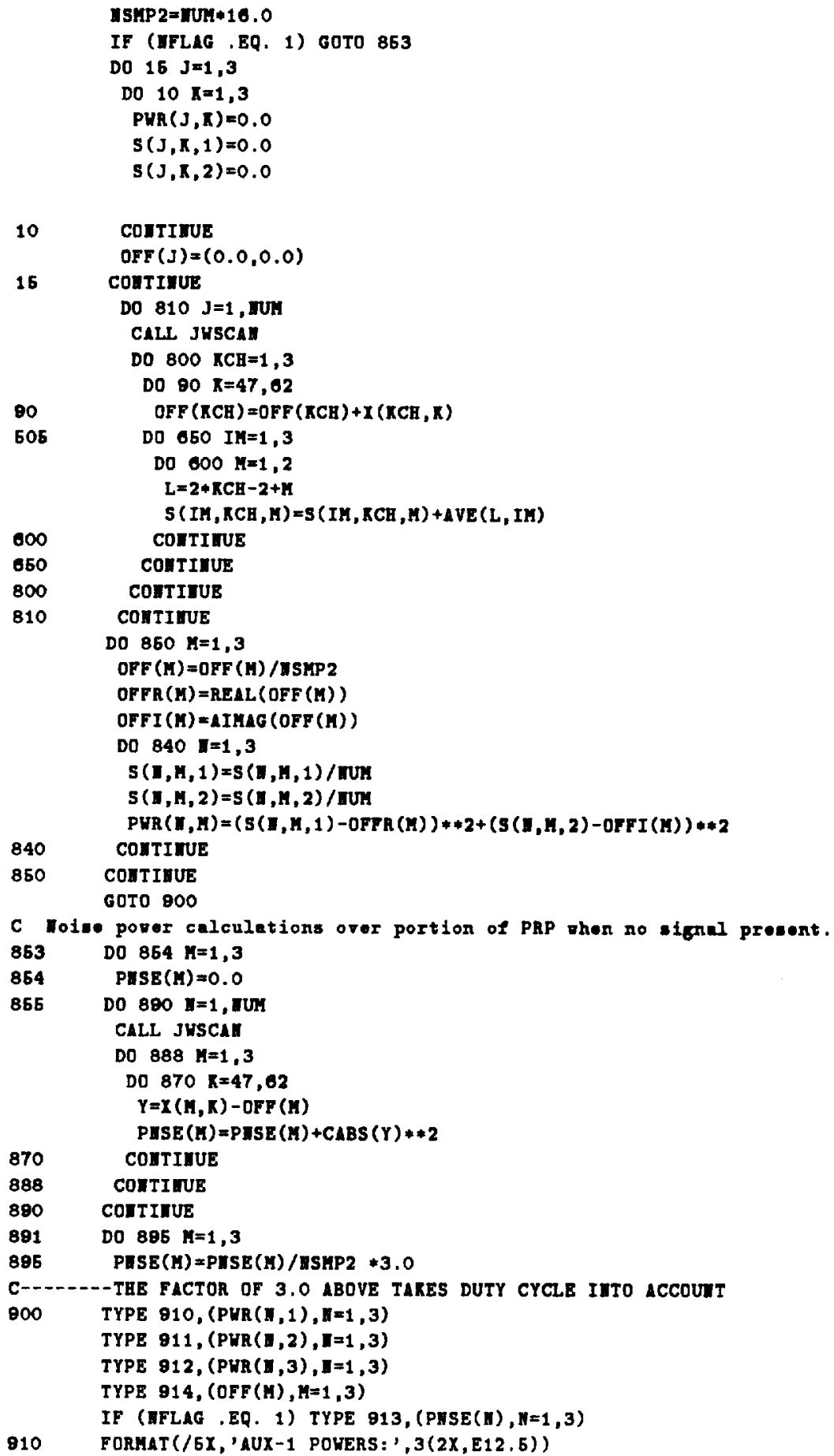




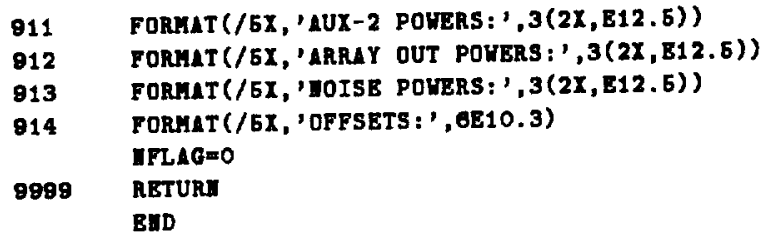




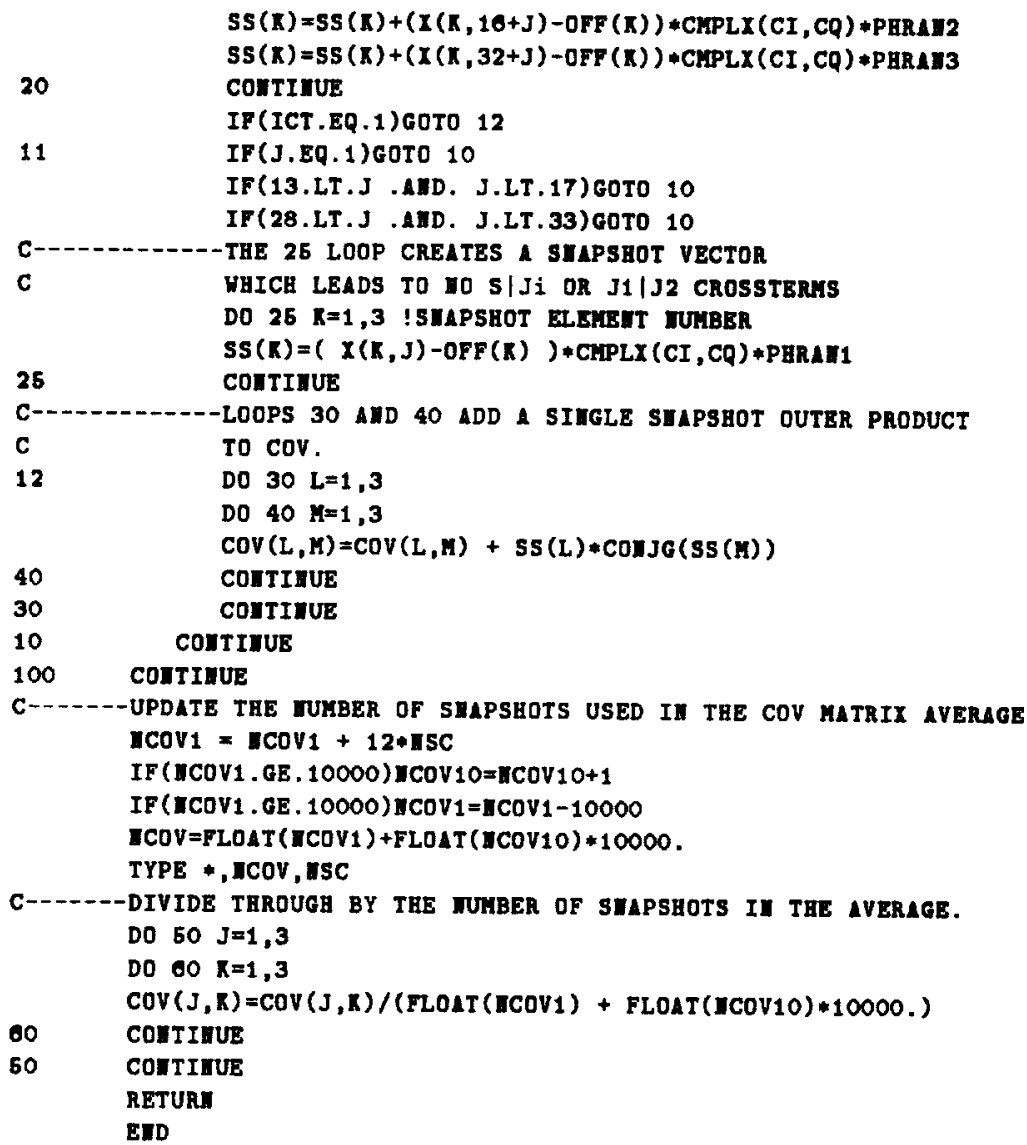

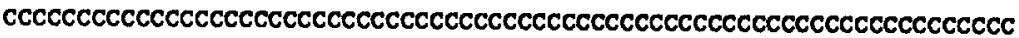

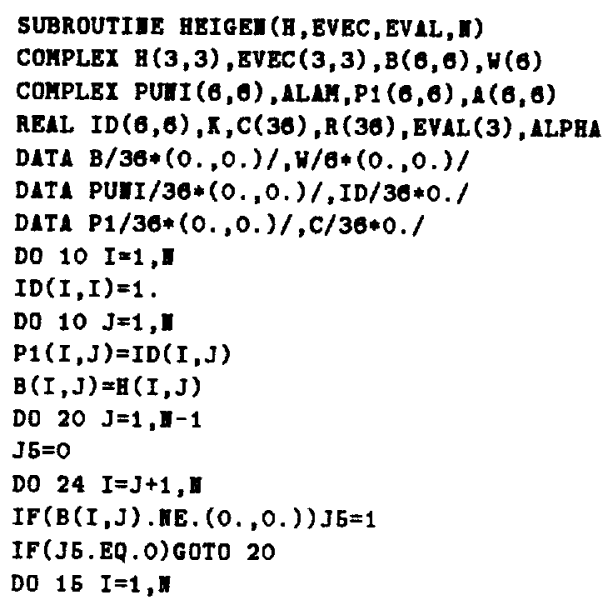


DO $16 \pi 1=1$, I

$\operatorname{PUII}(I, K 1)=I D(I, K 1)$

$\mathbf{R}=0$.

DO $30 \quad I=\mathrm{J}+1, \mathrm{I}$

30

$R=C A B S(B(I, J)) * 2+\pi$

$\pi=\operatorname{SQRT}(\boldsymbol{K})$

$J 6=0$

DO $150 \quad I=J+2$,

$\operatorname{IF}(B(I, J)$.WE. $(0,0)) J E=$.

$\operatorname{IF}((36, E Q .0)$.ADD. ABS $(R-R E L L(B(J+1, J)))$.LT. $0.1 E-10)$ COTO 20

ALPHA $A, * \pi * * 2-2, * \pi * \operatorname{REAL}(B(J+1, J))$

ALAM $=A L P H A /(R * 2-R * B(J+1, J))$

$U(J+1)=B(J+1, J)-x$

DO $50 \quad I=J+2, \pi$

bo $\quad W(I)=B(I, J)$

DO $\odot O I=J+1, \pi$

DO $60 \mathrm{~K} 1=\mathrm{J}+1$, $\mathrm{I}$

$60 \operatorname{PUII}(I, K 1)=I D(I, R 1)-($ ALAH/ALPHA $) * W(I) * \operatorname{CONJG}(W(R 1))$

DO $40 \quad I=1$, II

DO $40 \quad \times 1=1, n$

$40 \quad(\mathrm{I}, \mathrm{K} 1)=(0 ., 0$.

DO $100 \quad I=1$, I

DO $100 \mathrm{~L}=1, \mathrm{I}$

DO $100 \mathrm{M}=1, \mathrm{I}$

DO $100 \mathrm{M1}=1, \mathrm{~N}$

$100 \quad A(I, M 1)=\operatorname{PUMI}(I, L) * B(L, H) * \operatorname{COHJO}(P U M I(M 1, H))+L(I, M 1)$

DO $80 I=1,1$

DO $80 \times 1=1$,

$B(I, R 1)=\mathbf{A}\left(I, R_{1}\right)$

$80 \quad A(I, N 1)=(0 ., 0$.

DO $110 \quad I=1$,

DO $110 M 1=1$,

DO $110 \mathrm{M} 2=1$,

$110 \quad A(I, H 2)=P 1(I, M 1) * \operatorname{COMJG}(P U M I(M 2, M 1))+A(I$, H2 $)$

DO $90 \quad I=1, y$

DO $90 \mathrm{M} 1=1$,

O0 $\quad P_{1}(I, M 1)=A(I, M 1)$

20 CONTIAUE

$\mathrm{R} 1=1$

DO $70 \mathrm{I}=1$,

DO $70 \mathrm{~J}=1, \mathrm{I}$

$C(R 1)=\operatorname{REAL}(B(J, I))$

$70 \quad \mathrm{R} 1=\mathrm{K} 1+1$

CALL BIGEM(C, $R, \Pi, 0)$

$\mathrm{R} 1=1$

DO $130 \quad I=1,1$

DO $130 \mathrm{~J}=1$, I

$\operatorname{BVEC}(I, J)=\operatorname{CHPLX}(0.00,0.00)$

$\operatorname{PUnI} I(J, I)=R(R 1)$

$130 \quad \mathrm{~K}=\mathrm{K} 1+1$

DO $120 \mathrm{I}=1$,

DO $120 \mathrm{~J}=1, \mathrm{I}$

DO $120 \mathrm{M1}=1$,

$120 \operatorname{EVEC}(I, M 1)=\operatorname{P1}(I, J) * \operatorname{PUVI}(J, M 1)+\operatorname{BVEC}(I, M 1)$

DO $140 \quad I=1$, II 


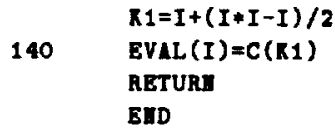




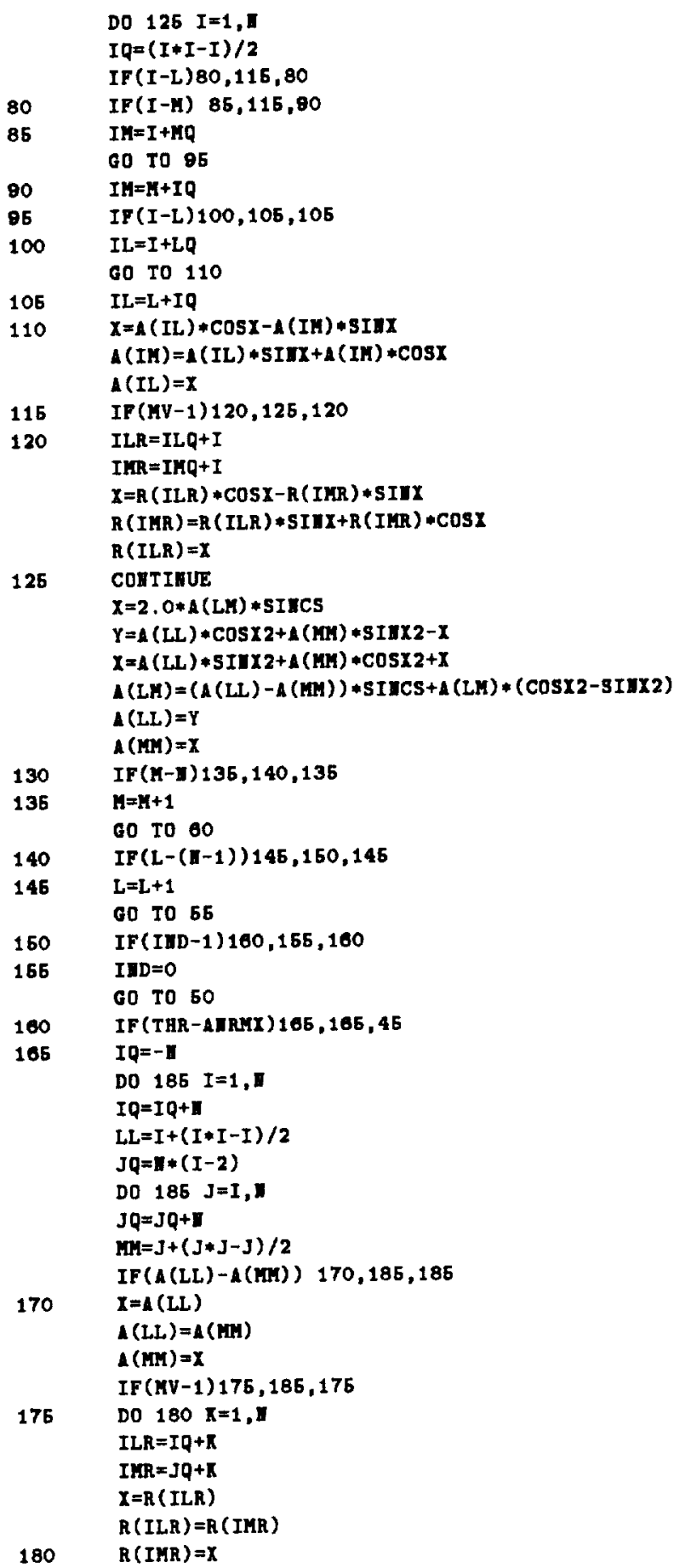




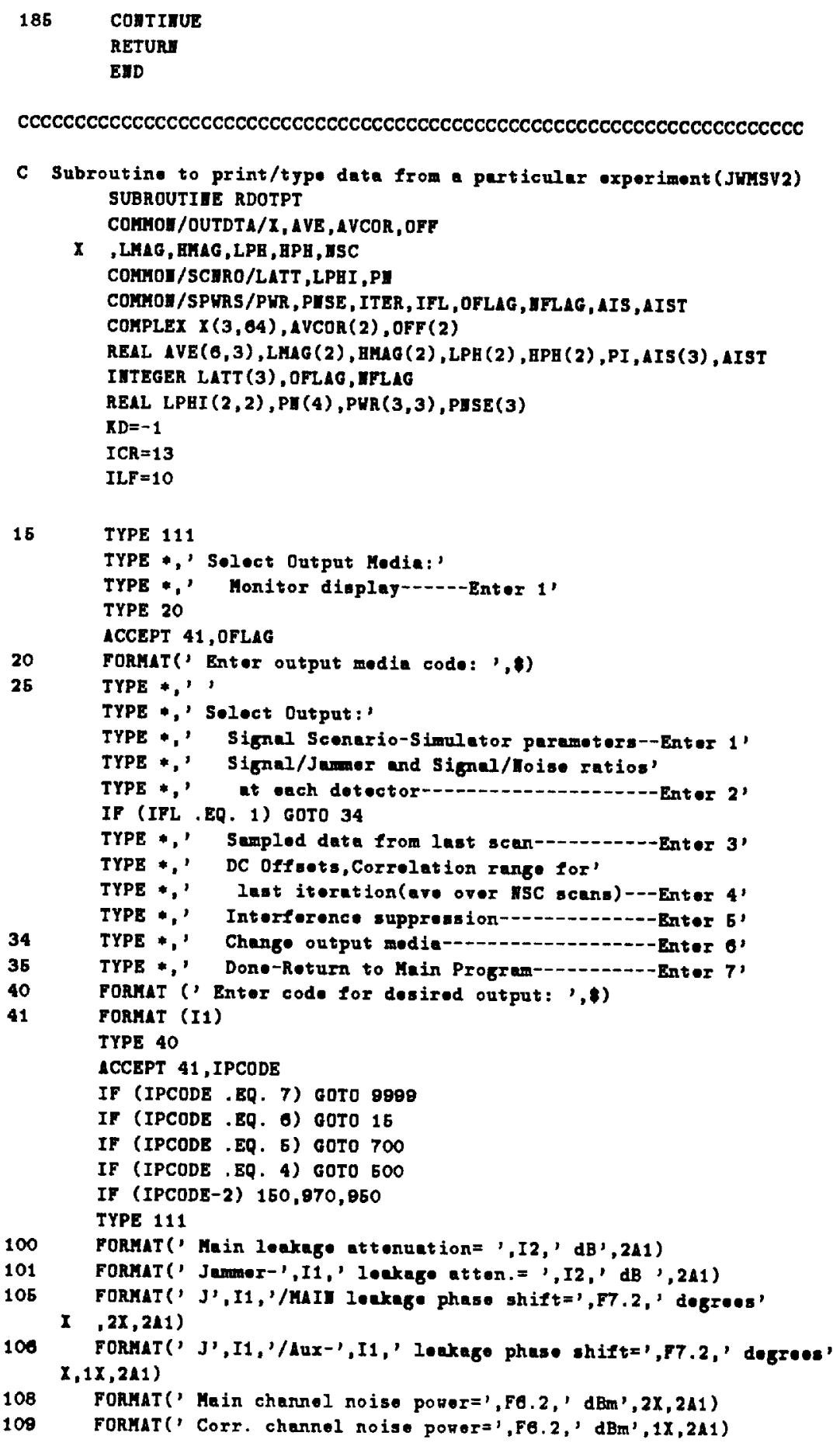




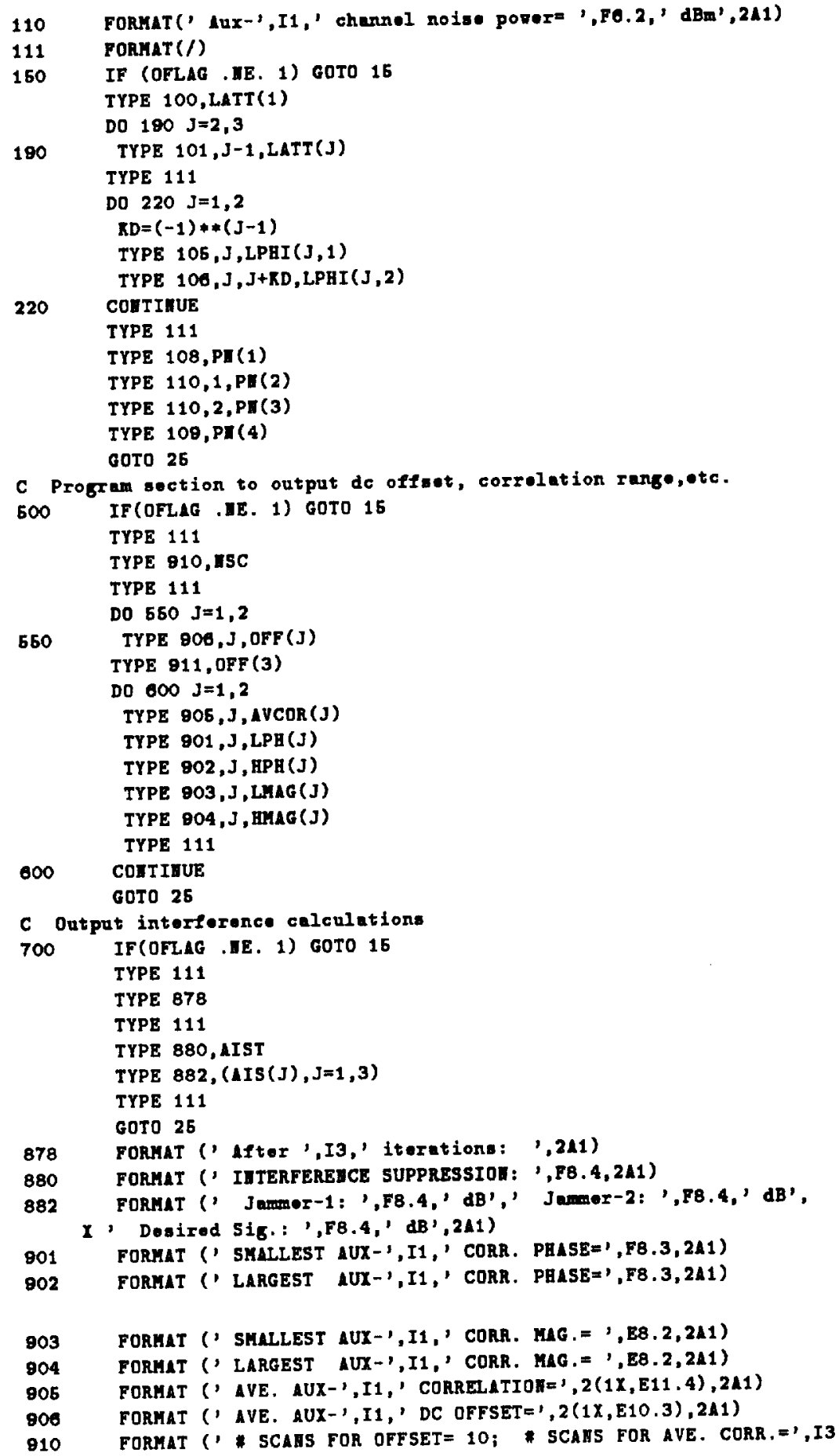


$x, 211)$

FORMAT (' IRRAY OUT DC OFFSET $=1,2(1 X, E 10.3), 221)$

TYPE*, 'I DISABLED THIS OPIION'

GOTO 25

970 CALL RDPRAT

IF (IFL .EQ. 1) GOTO 9989

GOTO 25

9999 IFL $=0$

RETURM

EID

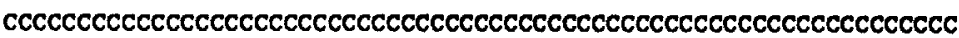

C Subroutine rdpret, to calculate and output the $s / J, s / 4$ ratios,

$c$ and simulated sidolobe lorols associated vith a given exper-

C Iment.

SUBROUTIITE RDPRAT

COMMOI/SPURS/PUR, PISE, ITER, IFL, OFLAG

IMTEGER IFL, OFLAG

REAL PUR (3,3), PISE (3), SLL (3), PRAT $(3,2), \operatorname{SIR}(3), \operatorname{SIR}$

$\operatorname{PRAT}(1,1)=10.0 * 1 \operatorname{LG} 10(\operatorname{PUR}(1,1) / \operatorname{PHR}(2,1))$

$\operatorname{PRLT}(1,2)=10.0 * 1 \operatorname{LG} 10(\operatorname{PHR}(1,1) / \operatorname{PUR}(3,1))$

$\operatorname{PRAT}(2,1)=10.0 * 1 L 0610(\operatorname{PUR}(2,2) / \operatorname{PUR}(1,2))$

$\operatorname{PRAT}(2,2)=10.0 * 1 \operatorname{LOG} 10(\operatorname{PUR}(2,2) / \operatorname{PUR}(3,2))$

$\operatorname{PRAT}(3,1)=10.0 * 1 L 0 G 10(\operatorname{PUR}(3,3) / \operatorname{PUR}(1,3))$

$\operatorname{PRAT}(3,2)=10.0 * 1 L 0 G 10(\operatorname{PUR}(3,3) / \operatorname{PUR}(2,3))$

$\operatorname{SIR}=10.0 * A L 0 G 10(\operatorname{PUR}(3,3) /(\operatorname{PHR}(1,3)+\operatorname{PWR}(2,3)))$

$\operatorname{SLL}(1)=\Lambda M I M 1(\operatorname{PRAT}(1,1), \operatorname{PRAT}(1,2))$

$\operatorname{SLL}(2)=\operatorname{AMIM1}(\operatorname{PRAT}(2,1), \operatorname{PRAT}(2,2))$

$\operatorname{SLL}(3)=A M I M 1(\operatorname{PRAT}(3,1), \operatorname{PRAT}(3,2))$

C The factor -7.27 is due to the everage power of our pulsed

C sinusoids, Pare $=(A c * * 2) / 2 *($ duty eyclo). The quantity PUh $(I, I)$

represerts $(A c * * 2) / 2$. The duty eycle cancels when taking eignal to

signal ratios, but mast bo included for SllR computetiona.

UPDATE 8- $\theta-88$ BY DILSAVOR: THIS FACTOR IS TAKEI IHTO ACCOUIT

BY SCALING PMSE BY 3.0 as SOON AS IT IS CALCULATBD III JWISC

DO $18 \quad I=1,3$

$18 \operatorname{SHR}(I)=10.0 * \operatorname{ALOG} 10(\mathrm{PWR}(I, I) / \mathrm{PNSE}(I))$

C18 $\operatorname{SIR}(I)=10.0 * 1 \operatorname{LOQ10}(\operatorname{PUR}(I, I) / \operatorname{PISE}(I))-7.27$

C IF (OFLAG .HE. 1) GOTO 990

TYPE 899

IF (IFL .ER. 1) TYPE 898

49

DO $100 \mathrm{~J}=1,2$

$\mathrm{R}=(-1) * * \mathrm{~J}$

TYPE $900, \mathrm{~J}$

TYPE $901, J, J-\pi, \operatorname{PRT}(J, 1), J, \operatorname{PRAT}(J, 2), J, \operatorname{SZR}(J)$

100 CONTIMUE

TYPE 902

TYPE 903, PRAT $(3,1), \operatorname{PRAT}(3,2), 5$ SR(3)

TYPE 804, SIR

$\operatorname{RI1HRM}=\operatorname{SMR}(3)-\operatorname{PRAT}(3,1)$

RI 2 HRH $=\operatorname{SER}(3)-\operatorname{PRAT}(3,2)$

RLATT1 $=10.0$ *ALOG10( PUR $(1,1) / \operatorname{PUR}(1,3)$ )

RLATT2 $=10.0 * A L O G 10(\operatorname{PHR}(2,2) / \operatorname{PHR}(2,3)$

TYPE 905, RLATT1, RLATT2

TYPE 906, RI1 IRM, RI2HRM 


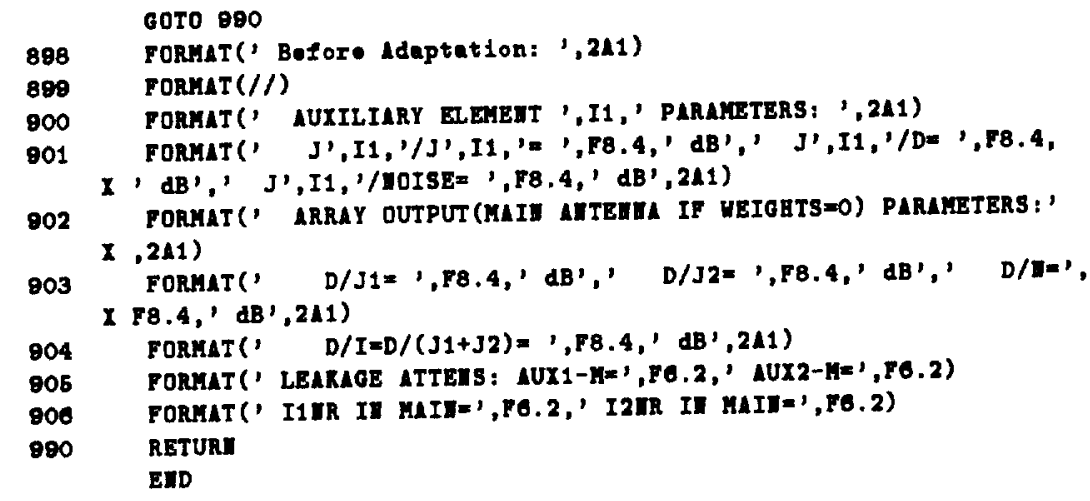




